\title{
Neuropathic pain : glial responses and plasticity in the spinal nociceptive network
}

Citation for published version (APA):

Jaken, R. J. P. (2010). Neuropathic pain : glial responses and plasticity in the spinal nociceptive network. [Doctoral Thesis, Maastricht University]. Maastricht University. https://doi.org/10.26481/dis.20101208rj

Document status and date:

Published: 01/01/2010

DOI:

10.26481/dis.20101208rj

Document Version:

Publisher's PDF, also known as Version of record

\section{Please check the document version of this publication:}

- A submitted manuscript is the version of the article upon submission and before peer-review. There can be important differences between the submitted version and the official published version of record.

People interested in the research are advised to contact the author for the final version of the publication, or visit the DOI to the publisher's website.

- The final author version and the galley proof are versions of the publication after peer review.

- The final published version features the final layout of the paper including the volume, issue and page numbers.

Link to publication

\footnotetext{
General rights rights.

- You may freely distribute the URL identifying the publication in the public portal. please follow below link for the End User Agreement:

www.umlib.nl/taverne-license

Take down policy

If you believe that this document breaches copyright please contact us at:

repository@maastrichtuniversity.nl

providing details and we will investigate your claim.
}

Copyright and moral rights for the publications made accessible in the public portal are retained by the authors and/or other copyright owners and it is a condition of accessing publications that users recognise and abide by the legal requirements associated with these

- Users may download and print one copy of any publication from the public portal for the purpose of private study or research.

- You may not further distribute the material or use it for any profit-making activity or commercial gain

If the publication is distributed under the terms of Article $25 \mathrm{fa}$ of the Dutch Copyright Act, indicated by the "Taverne" license above, 


\section{Neuropathic pain: \\ glial responses and plasticity \\ in the spinal nociceptive network}

Robby J.P. Jaken 
(c) Robby J.P. Jaken, Maastricht 2010

ISBN 978-90-9025712-9

Cover design, typesetting and layout: Michał Sławiński, Robby J.P. Jaken

Printed in Poland 


\title{
Neuropathic pain: glial responses and plasticity in the spinal nociceptive network
}

\author{
Proefschrift \\ ter verkrijging van de graad van doctor \\ aan de Universiteit Maastricht, \\ op gezag van de Rector Magnificus, \\ Prof. Mr. G.P.M.F. Mols,
}

volgens het besluit van het College van Decanen,

in het openbaar te verdedigen op

woensdag 8 december 2010 om 14.00 uur

door

Robby Jean Paul Jaken 


\section{Promotor}

Prof. dr. M. Marcus

\section{Copromotores}

Dr. R. Deumens

Dr. E. Joosten

\section{Beoordelingscommissie}

Prof. dr. M. de Baets (voorzitter)

Prof. dr. A. Dahan (Leiden University Medical Center, Nederland)

Prof. dr. R. van der Hulst

Dr. R. van Oostenbrugge

Prof. dr. J. Weis (RWTH Aachen University Hospital, Duitsland) 
It is easier to find men who will volunteer to die, than to find those who are willing to endure pain with patience Julius Caesar 



\section{Contents}

\section{Chapter $1 \quad 11$}

General introduction

\section{Chapter $2 \quad 21$}

Cellular and molecular insights into the chronic and intractable nature of neuropathic hypersensitivity

\section{Chapter $3 \quad 55$}

The CatWalk gait analysis in assessment of both dynamic and static gait changes after adult rat sciatic nerve resection

\section{Chapter $4 \quad 77$}

Synaptic plasticity in the substantia gelatinosa in a model of chronic neuropathic pain

\section{Chapter $5 \quad 85$}

Inverse relation between intensity of GFAP expression in the substantia gelatinosa and degree of chronic mechanical allodynia

\section{Chapter $6 \quad 97$}

Nociceptive fiber sprouting and neuropathic pain: activation of a silent circuit?

\section{Chapter $7 \quad 121$}

General discussion

137

Summary

139

Samenvatting

\section{1}

Dankwoord

\section{3}

Curriculum Vitae

\section{5}

List of publications 



\section{Abbreviations}

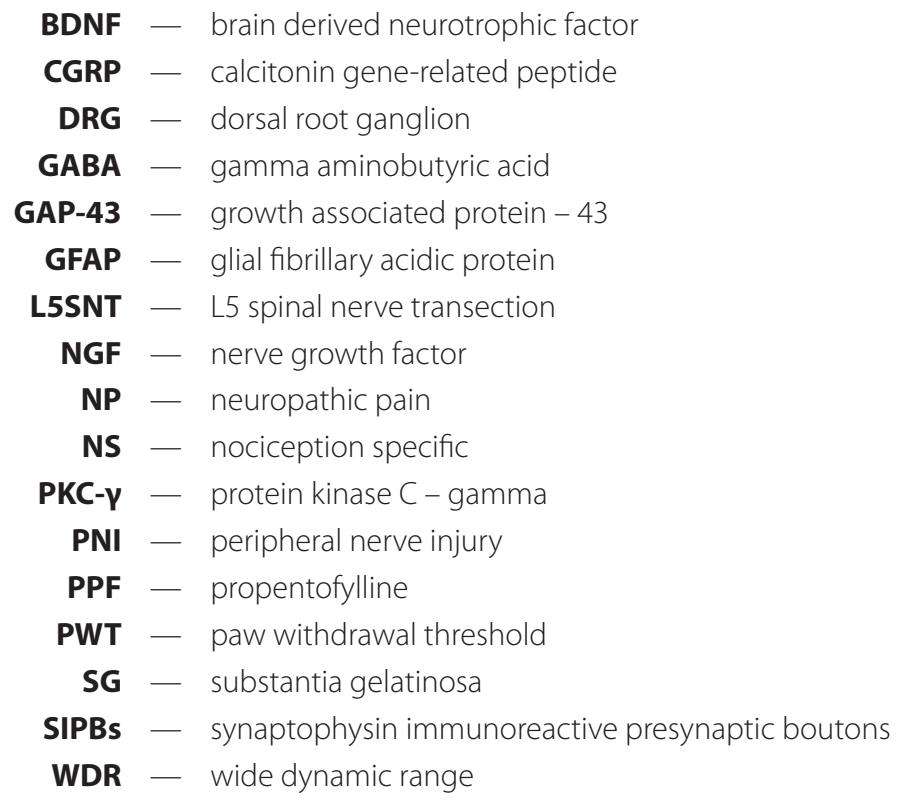



General introduction 



\section{Neuropathic pain: the clinical situation}

The International Association for the Study of Pain (IASP) has defined neuropathic pain as "pain initiated or caused by a primary lesion or dysfunction in the central or peripheral nervous system" [1]. Recently, neuropathic pain had been redefined as "pain arising as a direct consequence of a lesion or disease affecting the somatosensory system [2]. Central neuropathic pain may among others result from stroke, multiple sclerosis or spinal cord injury, while peripheral neuropathic pain may be caused by for example peripheral nerve injury, radiculopathies, or diabetic neuropathy [3]. It has been clear that neuropathic pain often presents with a wide range of symptoms in the clinical condition. Neuropathic pain symptoms include spontaneous, stimulus-independent types of pain, including sensations of burning, tingling, shock-like sensations. Moreover, stimulus-dependent types of pain (hypersensitivity) like allodynia (pain caused by a normally non-painful stimulus) and hyperalgesia (an exaggerated response to a normally painful stimulus) often manifest in patients.

The diversity in the underlying causes of neuropathic pain hinders reliable epidemiological studies. However, several studies report on the epidemiology of neuropathic pain in specific diseases. Of all diabetic patients, 20-24\% develops neuropathic pain [4], whereas complex regional pain syndrome patients display allodynia in $40 \%$ of the cases [5]. Pain experienced by cancer patients was shown to be of neuropathic nature in 33\% of the patients [6]. These numbers do not only indicate the extent of the problem, but also the impact on health care costs [7]. Although neuropathic pain patients often present with typical clinical features [8], the underlying mechanisms of characteristic pain states like paresthesias and/or allodynia are not always clear. As neuropathic pain is a complex and heterogeneous condition, limited therapeutic options exist. An ideal option would be a treatment which prevents neuropathic pain development, or perhaps more importantly, reverses established neuropathic pain. Nevertheless, incomplete knowledge of the mechanisms underlying neuropathic pain drives the clinician to treat the symptoms rather than their initial cause.

Pharmacological interventions are often the first choice of treatment, although several other strategies exist, like physical therapy, surgical interventions and electrical stimulation of peripheral nerves, dorsal root ganglia, spinal cord or the brain. Evidence-based guidelines for the pharmacological treatment of neuropathic pain have been described by the Neuropathic Pain Special Interest Group (NeuPSIG) of the International Association for the Study of Neuropathic Pain (IASP) [9]. These guidelines have put forward specific pharmacological treatments as first-line medications, which include tricyclic antidepressants (TCAs) often in combination with serotonin and norepinephrine reuptake inhibitors (SSNRIs), anticonvulsants including gabapentin and pregabalin, and topical lidocaine. Patients not responding to these first-line medications are generally treated with second-line medication like opioids and tramadol. Third-line medication, e.g. antiepileptic drugs, is reserved for patients not tolerating, responding to, or contraindicative for first- or second-line treatments. Other guidelines largely agree with the aforementioned recommendations $[10,11]$. 
Although some patients benefit from the aforementioned pharmacological treatments, a large number of neuropathic pain patients display relatively poor responses to current anti-pain medication and often experience severe side effects [12]. The limitations of current pharmacotherapies request further investigations in the mechanisms underlying neuropathic pain to develop adequate anti-pain medication. Animal models are valuable tools to investigate these underlying mechanisms and to test possible pharmacological therapies.

\section{Animal models and neuropathic pain symptoms}

Animal models for neuropathic pain are often highly reproducible and mimic clinical symptoms of neuropathic pain. Clinically, chronic neuropathic pain can be defined as neuropathic pain persisting for at least three months [13]. The present thesis was focused on two issues: neuroplasticity and glial responses in the spinal nociceptive network (1) late (i.e. at three months) following nerve injury and (2) early (i.e. within the first weeks) following nerve injury. The late time point was selected to investigate whether neuroplasticity and glial changes may contribute to the chronicity of neuropathic pain. The early time points were studied to gain insights into the mechanisms possibly resulting in the intractable nature of neuropathic pain and initiating the neuroplastic changes seen at chronic stages of neuropathic pain. The work described in this thesis is based on the use of two animal models differing in the temporal occurrence of neuropathic pain symptoms (i.e. allodynia and/or hyperalgesia), characterized by either a quick onset of neuropathic pain symptoms or a persistency of such symptoms beyond three months.

The first animal model used in our studies is complete transection of the sciatic nerve [14], characterized by mechanical hypersensitivity which persists for at least 10 months after initial injury [15]. Therefore, this model allows investigating the mechanisms contributing to the chronicity of neuropathic pain. Although signs of spontaneous pain-like behavior are observed from the first week post-injury, assessment of mechanical hypersensitivity within this early phase is not feasible due to loss of sensory function of the hind paw [15]. This latter aspect limits the use of the complete sciatic nerve transection model for investigations into the mechanisms involved in early phases of neuropathic pain.

However, complete transection of the sciatic nerve may be associated with long-term deficits of motor functions. The degree of motor impairments in this model has not been investigated in great detail, and a severe degree of motor impairments may strongly interfere with outcome measures of pain-assessment tests in rats, which are heavily based on paw withdrawal reflexes. Therefore, insights into the degree of persistent deficits of motor functions associated with complete sciatic nerve transection may be of particular interest for the interpretation of pain data obtained using this animal model of chronic neuropathic pain.

The second animal model used is this thesis is transection of the L5 spinal nerve, a modification of the L5/L6 spinal nerve ligation model [16]. This frequently used model al- 
lows investigating mechanisms involved in early phases of neuropathic pain because this highly accurate and reproducible model is characterized by the presence of mechanical hypersensitivity from day 1 post-injury onwards [16]. As the hypersensitivity resolves after about 2 months following initial insult [17], this model is unsuitable to investigate mechanisms involved in the chronicity of neuropathic pain.

\section{Behavioral testing tools to measure neuropathic pain symptoms in animal models}

Clinical symptoms of neuropathic pain can be measured in animals, in which hypersensitivity towards external stimuli is quantified by measuring paw withdrawal reflexes. Additionally, active attention towards applied stimuli including paw licking, postural changes, and/or attacking of the stimulus indicates the adversiveness against external stimuli, a behavior which goes beyond merely withdrawal reflexes. The von Frey test is used to assess hypersensitivity to mechanical stimuli through the application of filaments of varying thickness on the plantar surface of the hind paws $[18,19]$. This method has also been automated, in which increased pressure over time is applied to the hind paw (Dynamic Plantar Aesthesiometer, Ugo Basile, Italy). Withdrawal of the hind paws at a given stimulus or pressure is determined as the paw withdrawal threshold. The Hargreaves methods was designed to measure thermal hypersensitivity via a infrared beam, in which the time needed for the animal to withdraw its paw from the noxious thermal stimulation is regarded as the paw withdrawal latency [20].

Complete transection of the sciatic nerve may be associated with changes in motor function of the hind paws, which may possibly affect outcome measures of the von Frey and Hargreaves tests. The CatWalk method, an automated and objective gait analysis system, provides information regarding motor function. As paw withdrawal reflexes require adequate motor function of the hind paw, the CatWalk method may allow insights in the reliability of outcome measures of the von Frey and Hargreaves tests.

\section{From nociception to neuropathic pain: focus on structural changes and glial responses}

Nociception has been defined as the molecular, cellular and systemic mechanisms which deal with the detection and processing of potentially harmful stimuli [21]. Perception of this pain-related information is an essential physiological mechanism to guard the body against further injury. Potential or actual tissue damage may be perceived upon strong stimulation of peripheral nerve endings in the skin, induced by mechanical (e.g. pinprick), thermal (e.g. hot water) or chemical (e.g. acid) stressors. From here, electrical impulses are conducted along the peripheral nerve axon towards specific termination regions in the spinal cord. The specific termination pattern of both myelinated and non-myelinated peripheral nerve fibers which convey innocuous and noxious information respectively towards the spinal dorsal horn in- 
dicates the importance of this spinal region in the processing of sensory information. Specifically, the most superficial layers of the dorsal spinal cord, comprising inhibitory/excitatory interneurons and nociception-specific projection neurons, are intimately involved in the processing of noxious information. More specifically, the most ventral part of the inner lamina II comprises PKCY-positive interneurons which are connected to nociception-specific projection neurons in lamina I. The resultant circuit is normally inactive as it is under glycinergic and/or GABAergic inhibitory control [22,23]. Deeper dorsal horn layers contain wide dynamic range neurons which process both innocuous and noxious information. Altogether, the spinal dorsal horn consists of a highly organized nociceptive network crucial in the processing of peripheral, sensory input. Within the nociceptive network, peripheral inputs are processed and further transmission of electrical impulses towards higher brain centres results in the actual perception of pain.

Current understandings of the mechanisms which turn nociceptive processing into neuropathic pain are reviewed in chapter 2 of this thesis. The subsequent chapters focus on investigations on the potential role of (structural) neuronal and non-neuronal changes within the nociceptive network as possible contributors in early and chronic neuropathic pain. Structural changes within the highly organized nociceptive network could have major impact on normal pain processing and may underlie symptoms of neuropathic pain. Increased levels of the synaptic marker synaptophysin were found within the nociceptive network 2 weeks following peripheral nerve injury [24], indicative for injury-induced synaptic changes. These synaptic changes may result from sprouting of primary afferents into the superficial dorsal horn $[25,26]$.

Non-neuronal changes may also be involved in both early and chronic neuropathic pain. Glial cells have been shown to be closely involved in the development and maintenance of neuropathic pain conditions $[27,28,29]$. Peripheral nerve injury is associated with a neuroinflammatory response, involving activation of microglia and/or astroglia, which contribute to the pathophysiology of neuropathic pain [30,31]. Although numerous studies reported on the pro-nociceptive roles of microglia and astroglia, limited evidence exists on their specific role in the chronicity of neuropathic pain states. Moreover, their possible role in mediating structural changes within the spinal nociceptive network remains to be investigated. 


\section{Research questions and outline of this thesis}

The research performed and described in this thesis aimed at investigating the role of synaptic plasticity and glial responses in early and chronic neuropathic conditions. To study these research questions, two different animal models were selected to investigate the mechanisms underlying two distinct phases of neuropathic pain states.

In this thesis, the following research questions were addressed:

1. What is the current understanding of mechanisms underlying neuropathy-induced hypersensitivity?

2. Are there indications for motor deficits associated with sciatic nerve transection, which can strongly interfere with paw withdrawal reflexes towards externally applied stimuli?

3. Do persistent structural neuronal changes and glial activation occur within the spinal nociceptive network and contribute to chronic neuropathic pain?

4. Do structural changes within the spinal nociceptive network occur early after nerve injury and contribute to the intractable nature of neuropathic pain?

5. Do activated glial cells trigger nerve-injury-induced structural changes within the spinal nociceptive network?

In Chapter 2, current understanding of the cellular and molecular mechanisms underlying the chronic and intractable nature of neuropathy-induced hypersensitivity is reviewed.

Chapter 3 describes the long-term changes in dynamic and static gait parameters following sciatic nerve transection using the CatWalk method. The results of this study provide important information regarding possible changes in motor function, which may interfere with paw withdrawal reflexes to externally applied stimuli.

Chapter $\mathbf{4}$ focuses on late synaptic changes within the spinal nociceptive network in chronic neuropathic pain conditions. Late glial activation in chronic neuropathic pain is described in Chapter 5 .

Chapter 6 illustrates the injury-induced early structural changes within the spinal nociceptive network. Moreover, the possible role of glial cells in mediating these structural changes is described. 


\section{References}

1. Merskey, B., Classification of chronic pain: descriptions of chronic pain syndromes and definitions of pain terms. 1994, Seatlle: IASP Press.

2. Treede, R.D., et al., Neuropathic pain: redefinition and a grading system for clinical and research purposes. Neurology, 2008. 70(18): p. 1630-5.

3. Jensen, T.S., et al., The clinical picture of neuropathic pain. Eur J Pharmacol, 2001. 429(1-3): p. 1-11.

4. Schmader, K.E., Epidemiology and impact on quality of life of postherpetic neuralgia and painful diabetic neuropathy. Clin J Pain, 2002. 18(6): p. 350-4.

5. Sandroni, P., et al., Complex regional pain syndrome type I: incidence and prevalence in Olmsted county, a population-based study. Pain, 2003. 103(1-2): p. 199-207.

6. Davis, M.P. and D. Walsh, Epidemiology of cancer pain and factors influencing poor pain control. Am J Hosp Palliat Care, 2004. 21(2): p. 137-42.

7. Berger, A., E.M. Dukes, and G. Oster, Clinical characteristics and economic costs of patients with painful neuropathic disorders. J Pain, 2004. 5(3): p. 143-9.

8. Gilron, I., et al., Neuropathic pain: a practical guide for the clinician. CMAJ, 2006. 175(3): p. 265-75.

9. Dworkin, R.H., et al., Pharmacologic management of neuropathic pain: evidence-based recommendations. Pain, 2007. 132(3): p. 237-51.

10. Attal, N., et al., EFNS guidelines on pharmacological treatment of neuropathic pain. Eur J Neurol, 2006. 13(11): p. 1153-69.

11. Moulin, D.E., et al., Pharmacological management of chronic neuropathic pain - consensus statement and guidelines from the Canadian Pain Society. Pain Res Manag, 2007. 12(1): p. 13-21.

12. O'Connor, A.B. and R.H. Dworkin, Treatment of neuropathic pain: an overview of recent guidelines. Am J Med, 2009. 122(10 Suppl): p. S22-32.

13. Apkarian, A.V., M.N. Baliki, and P.Y. Geha, Towards a theory of chronic pain. Prog Neurobiol, 2009. 87(2): p. 81-97.

14. Wall, P.D., et al., Autotomy following peripheral nerve lesions: experimental anaesthesia dolorosa. Pain, 1979. 7(2): p. 103-11.

15. Kingery, W.S. and J.A. Vallin, The development of chronic mechanical hyperalgesia, autotomy and collateral sprouting following sciatic nerve section in rat. Pain, 1989. 38(3): p. 321-32.

16. Kim, S.H. and J.M. Chung, An experimental model for peripheral neuropathy produced by segmental spinal nerve ligation in the rat. Pain, 1992. 50(3): p. 355-63.

17. Kim, D.S., et al., Profiling of dynamically changed gene expression in dorsal root ganglia post peripheral nerve injury and a critical role of injury-induced glial fibrillary acidic protein in maintenance of pain behaviors [corrected]. Pain, 2009. 143(1-2): p. 114-22.

18. Chaplan, S.R., et al., Quantitative assessment of tactile allodynia in the rat paw. J Neurosci Methods, 1994. 53(1): p. 55-63.

19. Dixon, W.J., Efficient analysis of experimental observations. Annu Rev Pharmacol Toxicol, 1980. 20: p. $441-62$ 
20. Hargreaves, K., et al., A new and sensitive method for measuring thermal nociception in cutaneous hyperalgesia. Pain, 1988. 32(1): p. 77-88.

21. Sandkuhler, J., Models and mechanisms of hyperalgesia and allodynia. Physiol Rev, 2009. 89(2): p. 70758.

22. Miraucourt, L.S., R. Dallel, and D.L. Voisin, Glycine inhibitory dysfunction turns touch into pain through PKCgamma interneurons. PLoS One, 2007. 2(11): p. e1116.

23. Miraucourt, L.S., et al., Glycine inhibitory dysfunction induces a selectively dynamic, morphine-resistant, and neurokinin 1 receptor-independent mechanical allodynia. J Neurosci, 2009. 29(8): p. 2519-27.

24. Chou, A.K., et al., Altered synaptophysin expression in the rat spinal cord after chronic constriction injury of sciatic nerve. Neurosci Lett, 2002. 333(3): p. 155-8.

25. Woolf, C.J., P. Shortland, and R.E. Coggeshall, Peripheral nerve injury triggers central sprouting of myelinated afferents. Nature, 1992. 355(6355): p. 75-8.

26. Hu, J., et al., Central sprouting of uninjured small fiber afferents in the adult rat spinal cord following spinal nerveligation. Eur J Neurosci, 2004. 20(7): p. 1705-12.

27. Watkins, L.R., E.D. Milligan, and S.F. Maier, Glial activation: a driving force for pathological pain. Trends Neurosci, 2001. 24(8): p. 450-5.

28. Milligan, E.D. and L.R. Watkins, Pathological and protective roles of glia in chronic pain. Nat Rev Neurosci, 2009. 10(1): p. 23-36.

29. McMahon, S.B., W.B. Cafferty, and F. Marchand, Immune and glial cell factors as pain mediators and modulators. Exp Neurol, 2005. 192(2): p. 444-62.

30. Romero-Sandoval, A., et al., A comparison of spinal lbal and GFAP expression in rodent models of acute and chronic pain. Brain Res, 2008. 1219: p. 116-26.

31. Raghavendra, V., F. Tanga, and J.A. DeLeo, Inhibition of microglial activation attenuates the development but not existing hypersensitivity in a rat model of neuropathy. J Pharmacol Exp Ther, 2003. 306(2): p. 624-30. 



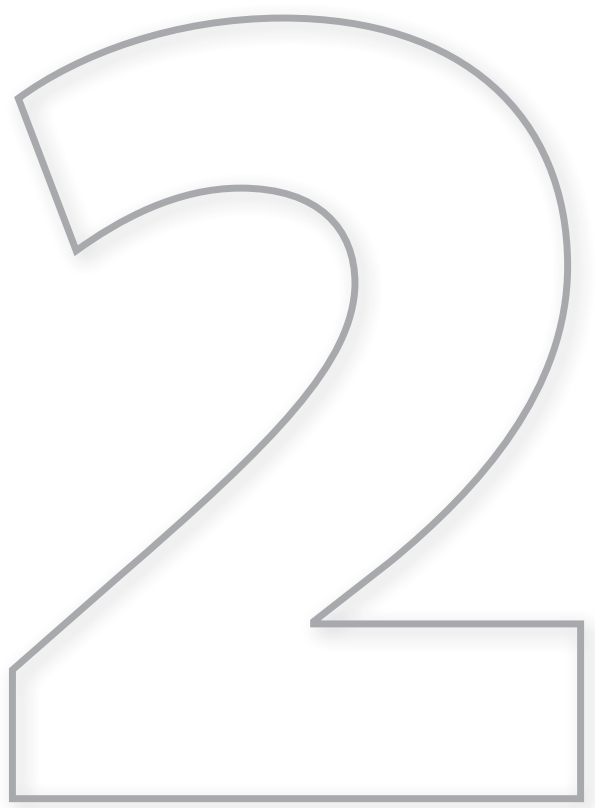

\title{
Cellular and molecular insights into the chronic and intractable nature of neuropathic hypersensitivity
}

\author{
R.J.P. Jaken*, L. Knaepen*, J. Berger, S.P.M. Janssen, \\ M.A.E. Marcus, E.A.J. Joosten, R. Deumens
}

Submitted 


\section{Abstract}

Neuropathic pain is not only the most prevalent type of chronic pain, but also particularly resistant to current pain medicine. Understanding of the pathological neuronal and non-neuronal mechanisms upstream of hyper-excitability of nociceptive networks and neuropathic hypersensitivity, but especially of the underlying 'causes' of these pathological mechanisms may aid in development of more effective neuropathic pain therapeutics. We here review proposed cellular and molecular mechanisms and show that (permanent) neurotrauma and related unremitting consequences such as ectopic firing, fiber sprouting, heat shock proteins and compromised bloodspinal cord barrier may give neuropathic pain its chronic nature. Neuropathic pain should be regarded as a disease rather than a syndrome and be treated as such. Although invasive pain management approaches including neurorepair or cell replacement therapies may still require some time before exploited clinically, novel pharmacotherapies are currently tested in clinical settings. Ideally, such pharmacotherapies should target both neuronal changes and specific pain-related glial 'response states.' Neurostimulatory treatments have shown to be particularly effective in treatment of neuropathic pain, and mechanisms-of-action are currently investigated to further extend beneficial effects. 


\section{Introduction}

Neuropathic pain represents one of the most debilitating disorders of mankind, and follows injury and/or dysfunction of the peripheral nervous system (PNS) or central nervous system (CNS). The prevalence of neuropathic pain depends on the related trauma/dysfunction, with numbers up to $30-50 \%$ of people with diabetic neuropathy and postherpetic neuralgia $[1,2]$, about $30 \%$ of spinally injured people, and virtually all people with radiculopathies representing the largest cause of chronic pain [3,4]. Neuropathic pain is perhaps the most common cause of chronic pain and management of this condition is still very unsatisfactory. A surge of investigations in animal models of neuropathic pain has unveiled mechanisms which may explain the chronic nature of neuropathic pain, and these insights are now thought to pave the road by which innovative, promising therapeutic interventions can and have made it to the clinical arena.

There are many pharmacological treatments for neuropathic pain, but these give limited, if any, therapeutic benefit and patients may show tolerance and/or unwanted sideeffects (see for an extensive review $[5,6]$. Despite the availability of a wide range of treatment options, neuropathic pain is still regarded as a chronic and intractable condition, which requires better management. A detailed view on the pathophysiological processes which can be observed in animal models of neuropathic pain have now provided clues about what makes this debilitating condition chronic and intractable. These insights may aid in the development of novel, more effective approaches for neuropathic pain management.

\section{Modeling neuropathic pain in rodents}

A variety of rodent models exist for investigations into neuropathic pain. In most of these models trauma is induced to either the PNS or CNS. Injury to the PNS mostly involves ligation and/or transection of (branches of) peripheral nerves, spinal nerves, spinal roots or dorsal root ganglia (DRG). The most frequently used models include ligation/transection of the $L 5$ and/or L6 spinal nerve (Chung model) [7], spared nerve injury (ligation/transection of the tibial and common peroneal branches of the sciatic nerve, leaving the sural branch intact) [8], partial sciatic nerve ligation (Seltzer model) [9], and chronic constriction injury (Bennett model) $[10,11]$. The common factor of these models is that only part of the sciatic nerve is injured. On the contrary, models of complete sciatic nerve injury (transection) are also used to study neuropathic pain [12]. Injury to spinal roots has been described as well as injury to DRG $[13,14]$. Injury to the CNS mostly involves spinal cord injury paradigms including spinal cord laceration/transection [15] and spinal cord contusion [16]. Mechanical and thermal hypersensitivity are observed in most of these animal models and used as the signs and symptoms to study neuropathic pain. Hypersensitivity can be characterized by enhanced 'pain-responses' to potentially harmful or noxious stimuli (i.e. hyperalgesia) and by 'pain-responses' to innocu- 
ous stimuli (i.e. allodynia), but in animal studies it is very difficult to discriminate between these two hypersensitivity symptoms. Interestingly it has been claimed that the term 'mechanical allodynia' should only be used when it is known that the stimulus does not elicit nociceptor activity, and hence is mediated via low-threshold afferent fibers [17].

Investigations of the spinal cord are of particular relevance in studies on neuropathic pain because the spinal cord is regarded as the 'gateway' to higher brain centers where information about location, affective and motivational information is integrated and related to the stimulus. Noxious and innocuous stimuli are transmitted to the spinal cord via non-myelinated/thinly-myelinated fibers (C-fibers and AS-fibers from small-diameter and mediumdiameter dorsal root ganglion (DRG) neurons or nociceptors) and highly-myelinated fibers (Aß-fibers from large-diameter DRG neurons), respectively. The termination pattern of these fibers in the spinal dorsal horn is highly organized as reviewed elsewhere [18]. Nociceptors specifically innervate the superficial laminae (lamina I, II and II), while A -fibers innervate deeper dorsal horn laminae or ascend to dorsal column nuclei in the brainstem immediately upon entering the spinal cord. The superficial dorsal horn contains many nociception-specific (NS) neurons projecting to supraspinal areas involved in processing of spatial, emotional, and affective aspects of nociception. In addition, inhibitory and excitatory interneurons, which are thought to modulate nociceptive signaling, reside in the superficial dorsal horn, particularly in lamina II: the substantia gelatinosa. The deeper dorsal horn (lamina V) contains widedynamic-range (WDR) projection neurons which respond to both noxious and innocuous peripheral stimuli. At present we are beginning to understand characteristics of the various dorsal horn neurons, functional connections and nociceptive circuits within the dorsal horn. This information will be vital for our understanding of nociception, and may shed more light on the processes underlying neuropathic pain symptoms such as hypersensitivity.

\section{Peripheral sensitization and hypersensitivity}

As the name already suggests, peripheral sensitization is the phenomenon in which peripherally located primary afferent neurons projecting to the spinal cord become hyper-excitable. Only a limited number of processes, occurring either at the DRG or at the peripheral nerve ending itself have been described to underlie the observed peripheral hyper-excitability. Most, but not all, of these changes involve changes in ion channels, and have been reviewed elsewhere [19]. Here, we focus on the main concepts which result in enhanced excitability of primary afferent neurons.

The cell bodies of primary afferent neurons are located in the DRG, where they are intermingled with satellite glial cells. The neurons and surrounding glial cells form distinct functional units [20]. Here, intercellular communication occurs extensively via non-synaptically released diffusible messengers such as ATP and this may modify the responsiveness of primary afferent fibers [21-23]. As such both neuronal and non-neuronal changes in the DRG 
may contribute to neuropathic hypersensitivity $[23,24]$. The purinergic receptors P2X3 and P2X7 may be involved in this process as they are expressed by both satellite cells and DRG neurons, particularly small diameter sized neurons [23,25-28]. Interference with these receptors attenuates or even prevents neuropathic hypersensitivity [29-31]. Nevertheless, since these purinergic receptors are not only expressed in the PNS but also in the CNS, it remains unclear how important the peripherally located purinergic receptors are in mediating neuropathic hypersensitivity. Also the fact that antagonist drugs for purinergic receptors are still largely unspecific and rapidly degrade does not help in understanding the role of specific purinergic receptor in neuropathic hypersensitivity [32]. In addition, nerve injury triggers the expression of chemokine receptors in DRG neurons. The monocyte chemotactic protein-1 or also termed CCL-2 and its receptor CCR2 are up-regulated in the DRG following nerve injury [33]. The fact that this molecular change may add to the excitability of primary afferent fibers is suggested by findings that CCL-2 can induce membrane depolarization of DRG neurons previously exposed to chronic constriction injury while these neurons are normally non-responsive to CCL-2 [34]. Then, the expression and/or phosphorylation status of receptor proteins such as the transient-receptor-potential-vanniloid-1 receptor (TRPV1) potentially relate to sensitization of peripheral nerve fibers. However, absence of functional TRPV1 receptors was shown to not prevent mechanical nor thermal hypersensitivity following peripheral nerve injury [35]. While ligands and receptors are changed in the DRG following nerve injury, the same holds true for intracellular signaling molecules such as mitogen-activated protein kinases (MAPK). Indeed, the phosphorylation of p38 MAPK and thereby its activation is increased in the DRG following nerve injury, and inhibition of p38 phosphorylation attenuates neuropathic hypersensitivity [36-38]. Nevertheless, it remains largely unclear to which extent the molecular alterations observed in the DRG contribute to neuropathic hypersensitivity since drugs which interfere with such molecules are often administered intrathecally, thereby having free access to DRG as well as spinal cord.

Beside molecular changes also morphological changes are reported to occur at the level of the DRG under neuropathic conditions. These changes include fiber sprouting, but the relevance to peripheral hyper-sensitivity and neuropathic hypersensitivity is uncertain [39-41]. Finally, enhanced primary afferent hyper-excitability may arise from changes at the peripheral nerve endings such as morphological changes in the peripheral innervation pattern of afferent fibers. Indeed, skin denervation due to peripheral nerve injury has been shown to be followed by collateral sprouting of intact afferent fibers [42-44], but it is largely unclear whether this adds to the sensitization of primary afferent neurons.

A phenomenon which is of particular importance to the activity of peripheral afferent fibers, but cannot be captured under the term 'hyper-excitability' is that of spontaneous electrical activity. Due to axotomy, trafficking of voltage-operated ion channels through the nerve fiber is hampered at the level of injury, which results in insertion and, thus, clustering of these channels into the cell membrane at this site $[45,46]$. As a result, the electrogenicity of the nerve fiber is altered leading to pacemaker capabilities. This spontaneous electrical activ- 
ity is mostly referred to as ectopic fiber activity or ectopia because the electrical discharges originate typically in ectopic places such as the mid-nerve or at the level of the DRG in case of peripheral nerve fibers. Both the enhanced excitability of peripheral afferent neurons and their spontaneous activity have radical consequences on the projection territories of these neurons within the CNS.

\section{Central sensitization and hypersensitivity}

Nerve injury dramatically changes the way in which incoming signals are processed in the spinal cord. This is evidenced by a strong increase in expression of the early neuronal activation marker c-Fos in the dorsal horn upon low-threshold mechanical stimulation of the hindpaw ipsilateral to a nerve injury [47] (Jaken et al., submitted). As mentioned above, the dorsal horn contains two different types of projection neurons: NS and WDR. In models of neuropathic pain, WDR have been found to increase their firing patterns following innocuous and noxious stimulation of the skin [48-50]. Also, a higher number of neurons with WDR characteristics have been noted $[50,51]$. Together, these findings point towards two concepts. First, sensitization of WDR neurons leads to hyper-excitability: decrease in the activation threshold and increase of electrical activity. Second, NS neurons acquire characteristics of WDR neurons, and thus also becoming responsive to innocuous stimuli (see Figure 1). This functional switch from NS to WDR neurons may be evoked either by sprouting low-threshold fibers onto NS projection neurons or by sensitization/activation of silent dorsal horn circuits between lowthreshold fibers and NS projection neurons. These two concepts are likely induced by central sensitization triggered by enhanced excitatory input, reduced inhibitory input and/or modulation of input as discussed below.

\section{A 'silent' dorsal horn circuit: sensitization and activation}

The spinal dorsal horn contains a 'silent' circuit between low-threshold afferent fibers and NS projection neurons. Up to now, the composition of this circuit has been only partly described. Of vital importance to the functioning of this circuit are putatively excitatory interneurons in the most ventral part of inner lamina II, which express the $\gamma$-isoform of protein kinase $C$ $(P K C-\gamma)$. These interneurons receive A $\beta$ fiber innervation, and innocuous stimuli are thus able to activate PKC $-\gamma$ interneurons via A $\beta$ fibers signaling [52]. This information is not gated to NS projection neurons in the more superficial dorsal horn because PKC- $\gamma$ interneurons are under inhibition of glycinergic and gamma-amino-butyric acid (GABA)ergic neurons [53]. If activated, this circuit would gate innocuous stimuli to NS projection neurons and, thus, turn 'touch into pain'. Indeed, when glycine receptors were blocked by intrathecal delivery of strychnine in naïve animals, dynamic innocuous stimuli were found to evoke early activation 
(c-Fos expression) of NS projection neurons and signs of mechanical hypersensitivity [54]. Notably, inhibition of PKC $-\gamma$ as well as blockade of glutamate receptors of the N-Methyl-DAspartate (NMDA)-type prevented the activation of NS neurons following innocuous stimulation, showing the vital role of PKC- $y$ and NMDA receptors in the gating of A $\beta$ fiber input to NS projection neurons [54]. Hence, 'touch can be turned into pain' by means of activation and/or sensitization of a silent dorsal horn circuit containing PKC $-\gamma$ interneurons, thereby gating $A \beta$ fiber input to NS projection neurons.

This circuit has shown to be of vital importance in the onset of hypersensitivity following neuropathy. Mice lacking the PKC- $\gamma$ have absent mechanical hypersensitivity and attenuated thermal hypersensitivity following partial sciatic nerve ligation [55]. Importantly, innocuous tactile hindpaw stimulation strongly increases the expression of c-Fos within and above the PKC- $y$ layer following nerve injury (Jaken et al., submitted; $[47,55]$. Activation of the PKC- $\gamma$-containing circuit might result from 'central' sensitization due to a change in the balance between inhibitory and excitatory input. Central sensitization was first described by Clifford Woolf and has boosted a tremendous interest into the field of pain research [56]. It is now clear that neuronal and non-neuronal cells contribute to this phenomenon in which CNS neurons have a higher background activity and become hyper-responsive to incoming neuronal signals [57].

Enhanced excitatory input is thought to originate from ectopic fiber activity (as discussed under 'peripheral sensitization') and fiber sprouting (as discussed below); reduced inhibitory input may result from compromised inhibitory systems including inhibitory interneurons and descending inhibitory pathways (as reviewed elsewhere $[58,59]$ ). In addition, there are several lines of evidence that neuroactive modulators such as cytokines and chemokines can alter the balance between inhibitory and excitatory input and result in hypersensitivity in conditions of neuropathy. First, interleukin-1 $\beta(\mathrm{IL}-1 \beta)$ and tumor necrosis factor-a (TNF-a) potentiate excitatory synaptic transmission in substantia gelatinosa neurons (in spinal cord explants) induced by ionotropic glutamate receptor agonists AMPA and/or NMDA [60]. Also, IL-1 $\beta$ and interleukin-6 (IL-6) suppress inhibitory synaptic transmission induced by GABA and glycine [60], thus giving rise to disinhibition. Second, cytokines/chemokines can increase the expression and release of neurotransmitters by primary afferent fibers $[61,62]$. Third, intrathecal delivery of interleukin-1 $\beta$ (IL-1 $\beta$ ) or tumor necrosis factor-a (TNF-a) increases WDR activation in naïve animals [63]. Fourth, mechanical hypersensitivity has been observed following intrathecal injection of exogenous fractalkine (CXCL3), and IL-1 $\beta$ but not TNF-a $[63,64]$. Fifth, interference with cytokines or with proteases important for cytokine activation (by means of cleaving of the pro-peptide, rendering mature cytokines) attenuates hypersensitivity following both injury to the PNS [65] and CNS [66]. Cellular sources of endogenous cytokines/chemokines are both injured neurons and activated glial cells [33,67-70]. Indeed, glial cells and more broadly, the activated immune system, importantly contributes to neuropathic pain [71]. 


\section{Central immunity and neuropathy-induced hypersensitivity}

Neuroinflammatory responses are highly complex and are mediated by neuronal and nonneuronal cells which act in synergy following pathological cues [57]. Cytokines and chemokines are known to largely orchestrate the inflammatory communications between neurons, local glial cells and blood-borne inflammatory cells. Indeed, cytokines/chemokines and other neuromodulators are up-regulated in the spinal cord within hours to days following peripheral neuropathies [72-75] and injury to the CNS [76,77]. Other neuromodulators such as prostaglandins, nitric oxide and excitatory amino acids, released in the CNS during a neuropathyinduced inflammatory response which can contribute to hypersensitivity $[78,79]$. The view on neuropathic pain has seen a revolutionary shift when it was discovered that non-neuronal cells are not simple by-standers in the processing of sensory neurotransmission, but play an active role or even exacerbate the enhanced interneuronal signaling in pathological conditions such as neuropathies $[57,80]$. Oligodendroglia, astroglia, and microglia constitute the major non-neuronal cell types in the CNS, but also blood-borne cells can invade the CNS, even after injury to peripheral nerves [81,82]. Astroglia and microglia are considered to largely influence the signal conduction between neurons [83]. Astroglia are resident in the adult CNS in two main forms: protoplasmic astroglia in the grey matter and fibrous astroglia in the white matter. Their intimate association with blood vessels gives rise to the blood-CNS barrier, and their location around synapses (giving rise to the so-called tripartite synapse $[84,85]$ ) enables astroglia to handle neurotransmission, e.g. by buffering glutamate at glutamatergic synapses. Microglia, on the other hand, are found around blood vessels (perivascular microglia) and within the CNS parenchyma. Microglia have a main role in immune surveillance as they continuously screen the CNS environment and can rapidly respond to disturbances in homeostasis $[86,87]$.

Following neurotrauma, glia undergo morphological transformations which are typically characterized by hypertrophy and an associated upregulation of glial-fibrillary-acidic protein (GFAP) for astroglia [88] and complement-receptor-3/CD11b [89] or the ionized calcium binding protein Iba1 for microglia [90]. Indicative of microglial responses is rapid proliferation, retraction of cellular processes, and acquisition of an amoeboid phenotype $[86,87]$. Interestingly, this type of response is not only accounted for by resident microglia, but also by bone marrow-derived cells which invade the spinal cord and differentiate into microglia [81]. Following nerve injury morphological changes are first observed in microglia, then in astroglia, although the exact timing seems to depend on the model used. Spinal nerve injuries (ligation and/or transection) elicit microglial responses within a day, which is then followed by astrocyte responses a few days later [91]. Injuries of the sciatic nerve or its branches usually show microglial responses within a week, followed by astrocyte activation $[69,92,93]$. Although the morphological hallmarks of glial responses are usually used to define an 'activated state', it does not tell us anything about the functional state of the glia. In addition, it is clear that there 
is no 'single' activation state, but many different states can occur depending on the context the glia are in [94]. For a better understanding on the role of activated glial states in pathological pain, it has recently been suggested to use the term 'pain-related enhanced response states' if it is clear that the molecular phenotype of a glia influences pain processing [95]. Most research up to now has used the classical markers of glial 'activation', but several molecular alterations have been described which contribute to symptoms of neuropathic pain.

The 'response state' in which microglia release brain-derived-neurotrophic-factor (BDNF) has been found to be particularly important for hypersensitivity following peripheral neuropathy [96]. This has been elegantly demonstrated by experiments showing that intrathecal administration of BDNF triggers mechanical hypersensitivity, an effect which can be modeled by [1] intrathecal delivery of ATP-challenged microglia or [2] spinal nerve injury [97]. This effect was explained by a down-regulation of the potassium-chloride cotransporter KCC2, resulting in increased intracellular chloride levels and a subsequent reversal of the anion gradient of GABA-induced currents in lamina I neurons from a hyperpolarizing to a depolarizing one [98]. As such, microglial BDNF triggers disinhibition at the level of the spinal dorsal horn. A spinal cord injury-induced 'response state' of microglia contributing to mechanical hypersensitivity involves increases in the expression of cycloxygenases [78] and the related expression of prostaglandin E2. Prostaglandin release following spinal cord injury was shown to depend on microglia and blocking of the prostaglandin receptor E-prostanoid 2 (EP2) present on dorsal horn neurons was found to attenuate hypersensitivity to tactile and thermal stimulation of the hindpaws [79]. Notably, peripheral nerve injury does not trigger such a'prostaglandin-response state' in microglia [99].

Besides microglia, astroglia predominate the glial cell populations in the CNS. We are currently just beginning to understand the role of astroglia in neuropathy-induced sensitization of the spinal nociceptive network and hypersensitivity. Although data unequivocally show that astroglial responses to nerve injury are related to hypersensitivity, the nature of such astroglial 'response states' and the mechanisms linking them to enhanced pain signaling remain largely unclear. A prominent role of astroglia involves handling of extracellular glutamate, which can be achieved by the several glutamate receptors and transporters (GLAST and GLT-1) expressed on the astroglial membrane [100]. Nerve injury has been associated with a down-regulation of glutamate transporters, which may result in increased sensitization of spinal nociceptive network and subsequent neuropathic hypersensitivity [101,102]. Also, an enhanced expression of extracellular protease tissue type plasminogen activator (tPA) in astroglia has been suggested to contribute to mechanical hypersensitivity following nerve injury, although it cannot be ruled out that tPA activity in the DRG is (partly) involved in this effect [103]. Also, it remains unclear how tPA would enhance nociceptive signaling in the spinal cord under neuropathic conditions, although proteases are of known importance to activation of cytokines, which can modulate nociceptive signaling. The most striking link between astroglia and neuropathy-induced hypersensitivity relates to the involvement of the MAPK c-Jun-N-terminal kinase (JNK). Neuropathy-induced JNK phosphorylation in the 
spinal cord was specifically restricted to a subset of (about 30\% of) astroglia (although DRG neurons also showed enhanced JNK phosphorylation) [104]. Inhibition of spinal, but not DRG JNK phosphorylation strongly attenuated hypersensitivity following peripheral nerve injury [104]. Subsequent investigations convincingly showed that JNK phosphorylation in astroglia results in up-regulation of CCL-2, which can potentiate excitatory neurotransmitter signaling in dorsal horn neurons [105].

Although 'classical' glial responses have been observed for months after nerve injury [69,92], the persistency of glial pain-related 'response states' is unclear. Time-dependent changes in glial responses are, however, likely to occur. A recent investigation showed that astroglial GFAP levels in the substantia gelatinosa inversely related to the degree of mechanical hypersensitivity at three months following peripheral nerve injury [106]. This suggests that rather late astroglial response states can even be anti-nociceptive rather than pro-nociceptive. In this perspective, information about temporal aspects of glial responses is at least as important as a clear description of the cellular and molecular character of such response states.

\section{What makes neuropathy-induced hypersensitivity chronic and/or intractable?}

Neuropathy-induced hypersensitivity is, thus, due to a shift in the balance between excitatory and inhibitory input into the spinal dorsal horn. A net increase in this balance resulting from enhanced input or disinhibition can lead to sensitization of dorsal horn neurons which increase their firing properties and this may underlie activation of the PKC- $\gamma$-containing circuit between A $\beta$ fibers and NS projection neurons. Although many of the currently used pain killers preferentially target neuronal signaling, they seem to be rather ineffective in treatment of neuropathic pain symptoms. A vital question remains to be answered to find more effective medicine to treat neuropathic pain: what makes this condition so chronic and intractable? Are (some of) the abovementioned mechanisms of neuropathy-induced hypersensitivity persistent and unremitting and why so? The answer to this question brings us back to some of the mechanisms, and on looking more closely at the mechanisms behind the mechanisms. Or phrased differently: what is the underlying cause of the neuronal and non-neuronal changes which enhance pain signaling in the spinal cord in neuropathic conditions?

\section{'Neuronal causes'}

Enhanced excitation of the spinal cord following neuropathies can arise from an increase in descending facilitation [107]. In addition, both central and peripheral nerve injury results in ectopic fiber activity. Spontaneous electrical discharges can originate from ectopic places such as the mid-nerve or at the level of the DRG in case of peripheral nerve fibers and, with high probability, in the spinothalamic tract following spinal cord injury $[48,108]$. With respect 
to peripheral nerve injury it is known that ectopic fiber activity is carried mostly by A $\beta$ fibers, although C fibers also show ectopia [46]. The contribution of specific ion channels to this phenomenon is still largely unknown, although it is unlikely that a pathological change in only a single ion channel can account for neuropathy-induced hypersensitivity [109-113]. By any means, mechanical hypersensitivity following peripheral nerve injury is strongly reduced when ectopia was attenuated or even abolished from injured primary afferent fibers, while leaving uninjured primary afferent fibers unaffected [114]. This then may imply that the chronicity and intractability of neuropathic pain symptoms may be related to an ongoing and persisting ectopia, giving rise to a continuous barrage of electrical impulses to nociceptive networks in the spinal dorsal horn, which are consequently sensitized.

In addition to such persistent changes in electrical fiber activity, nerve fibers have also been reported to display growth responses following central and peripheral neuropathy. These growth responses are termed 'sprouting' responses and are often observed in ectopic places. Initial studies suggested sprouting A $\beta$ fibers into the superficial spinal cord normally exclusively innervated by nociceptive primary afferent fibers [115-120]. It is now known that bias in fiber tracings can account for this phenomenon [121], and single-A $\beta$-fiber tracing studies have now shown that $A \beta$-fibers never extend beyond the dorsal horn layer occupied by PKCY neurons [122]. Hence, there is currently limited, if any, concrete evidence supporting A $\beta$ fiber sprouting into superficial dorsal horn laminae, thereby gating innocuous information to NS projection neurons. Nevertheless, fiber sprouting has been reported in several neuropathic conditions including peripheral nerve injury and spinal cord injury. Fibers most frequently reported to sprout include nociceptive fibers [123,124], especially of peptidergic nature. Indeed, enhanced numbers of fibers expressing calcitonin-gene-related-protein (CGRP), often co-expressing growth-associated-protein-43 (GAP-43) can be found following experimental spinal cord injury $[125,126]$ and inhibition of CGRP attenuated mechanical and thermal hypersensitivity [10]. Recently, CGRP fiber sprouting has been found within and above the PKC- $\gamma$ layer following spinal nerve injury (paper submitted). These sprouting fibers, either displaying ectopic activity or not, may sensitize the local neuronal networks including the normally silent circuit between A fibers and NS projection neurons. Fiber growth is thought to be triggered by enhanced levels of growth factors [127], but central and also peripheral fibers are known to be sensitive to their own set of growth factors depending on the repertoire of receptor proteins they express [128,129]. Interestingly, peptidergic fibers expressing the tropomyosin-receptor-kinase (trk)-A are particularly sensitive to nerve growth factor (NGF), which is released following nerve injury $[130,131]$. Indeed, NGF can induce sprouting of nociceptive fibers [132]. Anatomical or structural changes within the spinal dorsal horn following nerve injury may be quite persistent, and indeed changes in synaptic profiles within the substantia gelatinosa have been reported even at three months following peripheral neuropathy [133].

Of interest, peripheral nerve injury has been shown to trigger a phenotypic switch in large sized diameter primary afferent neurons. This phenotypic switch involves a de novo 
expression of neuromodulators such as substance P, CGRP, and BDNF in large sized diameter neurons [134-138]. These neuromodulators are of particular relevance to sensitization of dorsal horn neurons (see also [56]).

Persistent spinal disinhibition may contribute to the chronic and intractable nature of neuropathy-induced hypersensitivity. Malfunctions of both segmental inhibitory systems (such as inhibitory interneurons) and non-segmental inhibitory systems (such as inhibitory pathways descending from supraspinal locations) have been shown under neuropathic conditions. We here depict the GABAergic system to illustrate disinhibition and its possible role in reduced inhibition of dorsal horn neurons/circuits and neuropathic hypersensitivity. GABAmediated inhibitory post-synaptic currents (IPSCs) of dorsal horn neurons triggered by primary afferent stimulation have been shown to be decreased following partial but not complete peripheral nerve injury $[139,140]$. These reduced GABA-IPSCs may result from [1] a shift of the hyperpolarizing to a depolarizing character of GABAergic transmission under conditions of neuropathy (as previously discussed) [98] and/or [2] attenuated release of GABA. With respect to the latter, primary afferent evoked GABA release is indeed attenuated following peripheral nerve injury [141]. Also, basal extracellular GABA levels have been shown to be decreased under neuropathic conditions [142]. Changes in GABA receptor expression are largely uncertain $[139,143,144]$ but, if present, are most likely downstream to altered GABA levels and therefore not considered in detail here. Since the electrophysiological properties of GABAergic cells are unaltered under neuropathic conditions [145], decreased extracellular GABA levels may be based upon reduced GABA production. Indeed, reduced expression of the rate-limiting enzyme in GABA synthesis, glutamate decarboxylase (GAD), present in isoform-65 and isoform-67, is reported in the spinal cord up to two weeks following peripheral nerve injury [139] and even more persistent following spinal cord injury (decreased levels up to at least six weeks post SCI) [146]. The 'causes' of reduced GAD expression remain unclear, although cytokines have been reported to influence the expression of GAD in nonneuronal tissue [147]. Alternatively, it has been argued that GABAergic cells are prone to apoptosis under conditions of neuropathy, but the evidence is not extensive $[140,146]$. It has even been argued that there is no change in the number of GABAergic cells in the spinal dorsal horn following nerve injury $[148,149]$. Hence, more insights into the 'causes' of reduced GAD expression may aid in understanding the persistency of this phenomenon and it contribution to the intractable nature of neuropathic hypersensitivity.

\section{'Microglial causes'}

Classical microglial responses to nerve injury have received by far more attention than astroglial responses and are characterized by their relatively fast onset and robust nature $[94,150]$. Microglia have a wide repertoire of receptor proteins on their cell membrane which enable them to exert their role in immune surveillance. Among these receptor proteins are the tolllike receptors (TLRs), which are up-regulated following injury to CNS and PNS $[151,152]$. Ge- 
netically modified mice lacking normal expression of TLR2, 3, or 4 showed a strongly impaired classical microglial response to peripheral nerve injury and, moreover, attenuated hypersensitivity [153-155]. These findings show that TLRs enable microglia to adopt a pain-related 'response state' following nerve injury. But what is it about peripheral nerve injury that triggers TLR activation in the CNS? TLR are transmembrane signaling proteins which recognize damage-associated molecular patterns present on pathogens, but also endogenous proteins such as heat shock proteins have been identified as activating molecules [156]. Heat shock proteins are particularly up-regulated by glia and neurons in response to stress or injury [157], most likely for the purpose of neuroprotection [158]. The contribution of TLRs to the chronicity of neuropathic hypersensitivity remains unclear at present, although an increased expression has been observed for several weeks in a rat model of spinal cord injury [152]. It has been suggested that TLR are not only important in triggering an inflammatory response to neurotrauma, but also in its shaping [159].

One of the key features of microglial responses to neuropathy is enhanced cell motility, a prerequisite for chemotaxis, and thus, intensified interactions with the local environment [86]. The expression of the nucleotide receptor, the purinoceptor P2Y12, on microglia is required for microglial motility [160-162], and interference with its expression or activation resulted in impaired mechanical hypersensitivity following spinal nerve injury [163,164]. As discussed earlier, specific glial 'response states' have been linked to hypersensitivity following peripheral or central nerve trauma. The microglial 'BDNF' phenotype as demonstrated of specific importance in peripheral neuropathic pain, depends on the expression of a specific purinoceptor, P2X4. P2X4 is a cation-permeable ion channel, which is activated upon ATP-binding [165]. Microglial P2X4 expression is highly upregulated following spinal nerve injury in the rat [166], and this receptor was recently shown to be vital for microglial BDNF release [167] via activation of the mitogen activated protein kinase (MAPK) p38 [168]. Importantly, interference with either P2X4, p38, or trkB (the functional receptor of BDNF) decreases mechanical hypersensitivity following peripheral nerve injury $[97,166,169]$. P2X4 expression can be triggered in microglia upon exposure to ATP. Of specific interest is the finding that delivery of ATP-triggered microglia into the intrathecal space of naïve rats induces hyper-excitability of WDR neurons and a transition of NS neuron into WDR neuron in the spinal dorsal horn [50]. However, the molecular pathways upstream of P2X4 signaling in microglia following peripheral nerve injury have been only partly elucidated. The extracellular matrix (ECM) protein fibronectin, acting through its integrin receptors, triggers a src kinase called Lyn [170], which is important nerve-injury-induced up-regulation of P2X4 receptors in microglia [171]. Fibronectin levels have been found to increase in the spinal dorsal horn following nerve injury [172]. It has been suggested that the increased spinal fibronectin levels come from blood plasma via a leaking blood-spinal-cord-barrier, which can be present up to months following nerve injury [173].

The microglial 'prostaglandin' phenotype has been of particular importance to hypersensitivity following spinal cord injury and recent studies have shown that the chemokine CCL21 is a prerequisite for the development of this specific glial 'response state' [108]. Blocking 
CCL21 signaling was found to decrease microglial responses and hypersensitivity following nerve injury and CCL21 expression could be experimentally induced by evoking over-activity in afferent nerve fibers [108]. From these findings we may conclude that neuropathy-induced microglial responses depend to a certain extent on neuronal activity, perhaps ectopia. Indeed, as mentioned before, microglial responses to peripheral nerve injury can be prevented by blocking ectopia [114]. But how then does this work? Nerve fiber activity does not only lead to calcium-dependent neurotransmitter release, but can also trigger calcium-dependent release of neuromodulators such as chemokines. Indeed, in vitro experiments have shown that CCL-2 can be released in a calcium-dependent manner from DRG preparations upon challenge with either potassium chloride or capsaicin [174]. Subsequent in vivo experiments showed that CCL-2 is predominantly produced in small diameter DRG neurons, transported to the central terminals and released upon high intensity nerve stimulation [175]. Microglia express the receptor for CCL-2, CCR-2, and interference with CCL-2/CCR-2 signaling prevents nerve-injuryinduced microglial responses and mechanical hypersensitivity $[175,176]$. These data show us that enhanced nerve fiber activity is of special importance in microglial responses following nerve injury. The microglial 'response state' following CCL-2/CCR-2 signaling has been studied in more detail and found to entail microglial release of the lysosomal protease cathepsin $\mathrm{S}$ [177], which upon release cleaves a membrane-bound form of the chemokine fractalkine from neuronal membranes. Expression of the fractalkine receptor is specifically microglial [178] and induces phosphorylation and thereby activation of p38 MAPK, which is linked to production and release of pro-inflammatory cytokines and BDNF (as discussed above) [95].

Further evidence for the importance of nerve fiber activity in microglial responses comes from studies in which such responses were absent after central or peripheral nerve injury when nerve fiber activity was blocked using local anesthetics $[79,179]$. Especially C fibers seem to be important mediators of glial responses [180]. Interestingly, application of local anesthetics could reverse microglial responses following central, but not after peripheral nerve injury $[79,179]$.

\section{'Astroglial causes'}

Classical astroglial responses to nerve injury are typically observed to follow microglial responses $[69,91]$, which has led to the cautious conclusion that microglial responses trigger astroglial responses. Indeed, astroglial changes including altered expression of glutamate receptors glutamate transporters can be triggered by microglial 'response states' $[181,182]$. A recent study has identified the importance of microglial expression of interleukin-18 (IL-18) in the classical astroglial response to nerve injury [183]. Interference with signaling between microglial IL-18 and IL-18 receptor on astroglia largely prevented GFAP upregulation and mechanical hypersensitivity following peripheral nerve injury [183]. However, it is known that astroglial responses after nerve injury do not necessarily require microglial responses. Root avulsion typically results in astroglial responses in the absence of clear microglial responses 
$[184,185]$. So what then can trigger astroglial response to peripheral and/or central nerve injury if it is not microglia? Astroglia are particularly sensitive to cytokines. Tumor necrosis factor-a (TNF-a) has been found to trigger the 'JNK-CCL2' astroglial response state involved in neuropathic hypersensitivity [105]. Also, a recent study showed that enhanced neuronal activity is required for astroglial responses to peripheral nerve injury [186] and an NMDA receptor blocker has been found to prevent astroglial responses [187].

\section{Novel treatments focused on the cellular and molecular mechanisms which may make neuropathy-induced hypersensitivity chronic and/or intractable?}

Effective management of neuropathic pain is susceptible to at least three interrelated issues: targeting the appropriate cellular/molecular cue(s), targeting at appropriate location(s), and targeting at appropriate time(s). From the above mentioned it can be distillated that nerve trauma or disease elicit a wide range of cellular and molecular changes at many levels along the neuraxis. This holds true for both peripheral and central neuropathies. Hence, the initial and trivial cause of neuropathic hypersensitivity is the nerve injury itself [188]. As such one may argue that neuropathic pain is a neurodegenerative disorder rather than a syndrome/ group of symptoms. Then, it also needs to be treated as such. 'Curing' of neuropathic pain might, then, require [1] replacement of lost neurons such as inhibitory interneurons or [2] neurorepair. For obvious reasons such non-conventional and invasive treatment options do not belong to first-line options. Nevertheless, there are concrete ideas on managing neuropathic pain invasively as discussed below. Alternatively, one may return to more conventional treatments such as pharmacotherapies, but this time with better therapeutic targets at appropriate locations and at appropriate timing. Finally, neurostimulatory therapies have shown to be rather effective in treatment of neuropathic pain, but the mechanisms behind these therapies remain largely a focus of future investigations.

\section{'Neural replacement or neurorepair'}

Neural replacement is a strategy adopted in several investigations. Transplantation of several cell types including GABAergic cells or serotonergic cells was indeed found to attenuate hypersensitivity following spinal cord injury or peripheral nerve injury [189-192], but the benefit on hypersensitivity required early intervention (within two weeks) [193]. Despite the fact that these promising findings could be reproduced with a human cell line [194], the clinical feasibility of cell transplantation remains unclear as it is highly invasive and potential, adverse side-effects are only poorly described. Although the use of certain cell lines may be of potential benefit in treatment of neuropathic pain [195], other cell transplantation paradigms have been reported to even trigger neuropathic pain [196]. The issue of cell transplantation for treatment of neuropathic conditions needs to be approached with care [197]. 
Neurorepair, in theory, may solve many neuropathological events triggered by nerve injury, including ectopic fiber activity and consequent events including central glial responses. Repair of the injured spinal cord has been attempted in many different ways, and some of those are particularly attractive for their effect on central glia. One example is the use of olfactory ensheathing glia, which may not only show beneficial effects on re-growth of injured fibers [198-200], but also integrate well with astrocytes without eliciting a pathological response state [201]. Notably, transplantation of olfactory ensheathing glia has also been shown to improve the efficacy of repair strategies for peripheral nerve injury [202,203]. Transplantation of autologous olfactory ensheathing glia has even made it to clinical trials [204].

\section{'Pharmacotherapeutic approaches'}

The rather low therapeutic effects of currently used pharmacotherapies in treatment of neuropathic pain have been suggested to relate to a certain extent to the neuron-centric action of these drugs. Novel treatment options have been suggested and include immune suppressive therapies such as cytokine receptor blockers (etanercept, infliximab, etc.) and glial inhibitors (minocycline, propentofylline, etc.), some of which are currently tested in clinical trials (see recent review [205]. However, it is clear that understanding of the exact molecular nature of glial 'response states' is needed to design drugs which selectively suppress 'painrelated enhanced response states' without affecting the physiological function of these glial cells. Also, it cannot be ruled out that glial 'pain-related decreased response states' exist next to glial 'pain-related enhanced response states', perhaps depending on the time after injury. Indeed, a recent study showed that at a relatively late time point after peripheral nerve injury (i.e. three months), one of the classical hallmarks of astroglial responses to nerve injury, i.e. GFAP expression, inversely related to mechanical hypersensitivity [106]. This suggests that astroglial phenotypes exist which are linked to suppression of hypersensitivity at this late time point after nerve injury. As such we can even better understand that we are standing at the beginning of an avenue which leads to an understanding of various glial 'response states', some of which relate (positively or negatively) to neuropathic hypersensitivity.

For peripheral neuropathic pain it is argued that CNS changes are downstream of PNS changes. Hence, pharmacotherapies directed against PNS changes such as ectopia may prevent or attenuate further sensitization processes and neuropathic hypersensitivity. Also for central neuropathic pain, ectopia contributes to subsequent sensitization processes including glial cell responses [79]. An excellent review has recently pointed out that ectopia is not only attenuated by therapies targeting the PNS, but also many drugs claimed to have their effect via actions on the CNS actually affect the DRG and ectopia (see also a recent review [46]). Such drugs include anticonvulsants (gabapentin), antidepressants (tricyclics), NMDA receptor blockers (ketamine), and even glial modulators (minocycline) [46]. For the issue of appropriate timing, we still have limited knowledge, but current data suggest that earlier intervention may render better outcome. It is of special note that local anesthetic block of 
an injured peripheral nerve, thereby silencing ectopia, could prevent central glial responses, but could not reverse already established responses [179]. Moreover, anatomical changes due to fiber sprouting may hamper effective neuropathic pain management and as long as the underlying cellular and molecular requirements remain unclear its effective prevention is not a feasible goal. Since CGRP fibers have mostly been found to sprout and are thought to require NGF for this phenomenon, anti-NGF treatment has been explored experimentally. Indeed, CGRP fiber sprouting in the spinal dorsal horn following experimental spinal cord injury was found to be reduced by anti-NGF treatment [125]. Since it remains largely unknown how important the phenomenon of fiber sprouting is in neuropathic hypersensitivity, it is not likely that therapies against this phenomenon will soon be clinically exploited. A recent investigation analyzed cerebrospinal fluid of people affected by neuropathic pain for changes in neurotrophic factors and found a lower amount of glial cell line derived neurotrophic factor (GDNF) when compared to the spinal fluid of control groups [206]. Interestingly, intrathecal GDNF administration has been previously found to prevent mechanical and thermal hypersensitivity in rats with a partial sciatic nerve ligation and even reverse this hypersensitivity when it was already established [207]. It has to be noted, however, that GNDF treatment was also found to attenuate ectopic fiber activity in this animal model [207].

Appropriate treatment locations may be diverse as pathological neuronal changes are observed along the neuraxis, including (first order) DRG neurons, (second order) spinal dorsal horn neurons, and (third order) thalamic neurons following both peripheral neuropathy [48,208-210] and central neuropathy [211-213]. Moreover, peripheral nerve injury triggers central glial responses at the spinal levels innervated by the affected nerve and at supraspinal locations $[164,214]$. The same holds true for injury to the CNS although glial responses can be even more spread-out. Glial responses following spinal cord injury are found at many spinal segments distance from the original spinal level of injury. Of particular note, blocking of microglial responses in the ventrolateral posterior nucleus of the thalamus following experimental spinal cord injury effectively attenuates established mechanical and thermal hypersensitivity [79].

\section{'Neuromodulation by neurostimulation'}

Neurostimulatory therapies may be regarded as an alternative non-pharmacological end line anti-pain therapy for neuropathic pain. Neurostimulation is performed via electrical stimulation of [1] peripheral nerves via transcutaneous or percutaneous electrical nerve stimulation [215,216], [2] DRG via (pulsed) radiofrequency stimulation [217], [3] spinal cord via epidural spinal cord stimulation [218], and/or [4] brain via deep brain stimulation, epidural motor cortex stimulation, and/or transcranial magnetic stimulation [219].

SCS is used as an endpoint treatment for several neuropathic conditions including painful diabetic polyneuropathy and complex regional pain syndrome (CRPS) with long-term beneficial effects on pain outcome [218,220-222]. In a randomized clinical trial it was shown 
that SCS rendered significantly lower pain scores in about $60 \%$ of patients with CRPS from one month after start of treatment onwards [218]. Insights into the mechanism-of-action of SCS may aid in finding additional treatments to extend the efficacy of SCS to the $40 \%$ of CRPS patients not responding to this treatment. Here it is of special interest that a higher degree of mechanically evoked hypersensitivity following neuropathy may predict a lower efficacy of SCS on pain reduction in both a preclinical and clinical setting $[223,224]$. Although animal studies have shown that SCS results in early neuronal activation in the stimulated spinal cord [225], it is not known which neurons are susceptible to stimulation. The most convincing theory of SCS involves an action of SCS on the GABAergic system. SCS was found to increase levels of GABA, while glutamate levels were decreased at the spinal level of stimulation [226]. As such, it may be argued that lack of SCS efficacy is related to too extensive loss of GABAergic interneurons. Indeed, SCS-induced levels of spinal GABA were higher in neuropathic rats which showed attenuation of mechanical hypersensitivity following SCS (i.e. SCS-responders) compared to neuropathic rats which did not show differences in mechanical hypersensitivity following SCS (i.e. SCS-non-responders) [142]. Interestingly, SCS-non responders could be changed into SCS-responders when SCS was combined with sub-therapeutic intrathecal dosage of the GABA-B receptor agonist baclofen [227]. These findings have formed the incentive to clinical trials in which intrathecal baclofen is regarded as an effective adjunct therapy for SCS in treatment of peripheral neuropathic pain [228,229].

Pulsed radiofrequency (PRF) stimulation of the DRG has been shown effective in treatment of painful radiculopathy $[217,230]$, but also here the mechanism of action remains largely unknown. In contrast to conventional continuous radiofrequency treatment, the pulsatile nature of PRF does not lead to thermal lesioning effects [231], although there are indications that even PRF can evoke tissue damage including myelin damage [232]. Continuous radiofrequency and PRF treatment have been found to induce prolonged inhibition of evoked synaptic transmission [233], but the mechanisms behind these effects remain unknown. Interestingly, a single PRF treatment of the L5 DRG of naïve animals increased expression of the early neuronal activation marker c-Fos at one week after treatment [234]. Further investigations are required to understand better the cellular and molecular processes induced during PRF, giving this treatment its beneficial pain reducing effects. Although radiofrequency treatments of the DRG are quite effective in reducing neuropathic pain, preclinical data indicate a potential therapeutic benefit of PRF when applied percutaneously in an animal model of peripheral neuropathic pain [235]. 

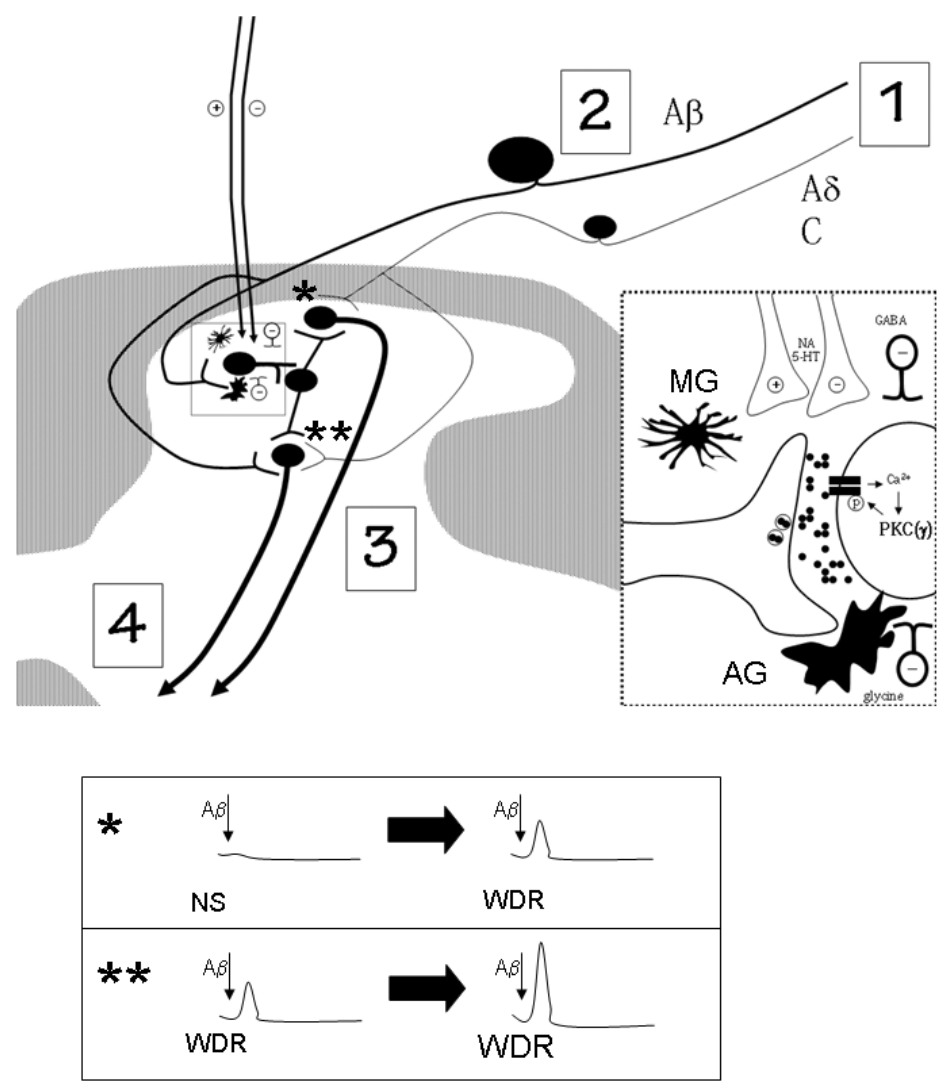

Figure 1. The spinal nociceptive network undergoes cellular and molecular changes in conditions of neuropathy. Innocuous and noxious stimuli trigger electrical signals in first-order large and medium/small sized diameter neurons, respectively; these signals are relayed to a rather complex nociceptive network in the dorsal horn of the spinal cord. This network receives distinct primary afferent innervation and contains segmentally restricted interneurons and projection neurons, the latter which relay either both innocuous and noxious stimuli (in case of WDR neurons) or noxious stimuli only (in case of NS neurons) to supraspial areas involved in somatosensation and/or pain perception. Signal transduction by projection neurons can be modulated at the level of the spinal cord by the activity of excitatory and inhibitory interneurons, descending pathways (releasing noradrenaline and/or serotonin) but also by glial cells including microglia (MG) and astroglia (AG). Putatively excitatory interneurons expressing the $\gamma$-isoform of protein kinase $C$ (PKC $\gamma$ ), are thought to be polysynaptically connected to projection neurons and play a prominent role in neuropathic hypersensitivity. Neuropathy-induced sensitization at the level of (1) free nerve endings in peripheral tissues, (2) dorsal root ganglia, (3) segmental dorsal spinal cord, and (4) projection neurons and/or supraspinal projection areas, can contribute to neuropathic hypersensitivity. As a result of sensitization, spinal projection neurons of the WDR type may become hyper-excitable and respond more strongly to incoming signals and/or projection neurons of the NS type may adopt WDR properties, thereby responding to normally innocuous stimuli such as $A \beta$ fiber input.

$A \beta, A \beta$-large-sized diameter neuron; $A \delta, A \delta$-medium-sized diameter neuron; $C, C$-small-sized diameter neuron; $M G$, microglia; $A G$, astroglia; P, phosphorylation; $\mathrm{Ca}^{2+}$, calcium entering the cell via e.g. NMDA receptor; $P K C \gamma$, protein kinase $C \gamma ; \mathrm{GABA}$, gamma-aminobutyric acid; NA, noradrenaline; 5-HT, 5-hydroxytryptamine/serotonin; NS, nociception-specific projection neuron; WDR, wide dynamic range projection neuron. 5-HT, 5-hydroxytryptamine/serotonin; NS, nociception-specific projection neuron; WDR, wide dynamic range projection neuron. 


\section{Concluding remarks}

The intractable nature of neuropathic pain suggests that currently used analgesic drugs target processes associated with symptoms rather than attacking the underlying causes. The chronic nature of neuropathic pain suggests that the actual cellular and/or molecular'causes' are not easily resolved spontaneously. We here showed that largely unremitting neurotrauma-related phenomenon such as ectopic firing/neuronal activity, fiber sprouting, a leaking blood-spinal-cord barrier, and enhanced expression of heat shock proteins may represent 'causes' of neuropathic hypersensitivity. 'Curing' neuropathic pain would then require repairing the injured nerve(s) and/or replacing lost neurons (such as inhibitory interneurons). As long as this approach is far from a clinical reality, more symptomatic approaches hold promise. Neurotrauma sets a large number of secondary processes in motion including glial changes which further exacerbate sensitization and consequently hypersensitivity. A defined set of glial 'response states' emerging following central or peripheral neuropathy have now been identified as related to neuropathic hypersensitivity. Besides further research to elucidate in more detail the cellular and molecular nature of glial pain-related response states, the logical next step is now to develop therapeutic agents which selectively target such response states. Therapeutics which may be particularly effective in attenuation of neuropathic hypersensitivity include pharmacotherapies targeting both neuronal changes and 'glial response states.' In addition to selection of (a set of) optimal therapeutic targets, location and timing of treatment designs need to be considered. With respect to location, the spinal cord dorsal horn may be of particular interest as nociceptive and non-nociceptive sensory peripheral input is integrated and processed here before information ascends into higher brain centers and actual pain perception occurs. Also, locations where ectopic fiber activity occurs are particularly qualified for treatment because ectopia is directly and indirectly related to hypersensitivity. Efficacy of pharmacological treatments is likely related to timing as well, but this area of research has clearly received only limited attention thus far. It can therefore only be recommended to extend knowledge on nerve-injury-induced cellular and molecular changes with respect to time after nerve injury. Finally, optimization of so-called endpoint therapeutics including neurostimulatory treatments may further improve its already beneficial effects on management of neuropathic pain. 


\section{References}

1. Veves, A., M. Backonja, and R.A. Malik, Painful diabetic neuropathy: epidemiology, natural history, early diagnosis, and treatment options. Pain Med, 2008. 9(6): p. 660-74.

2. Baron, R., et al., A cross-sectional cohort survey in 2100 patients with painful diabetic neuropathy and postherpetic neuralgia: Differences in demographic data and sensory symptoms. Pain, 2009. 146(1-2): p. 34-40.

3. Radhakrishnan, K., et al., Epidemiology of cervical radiculopathy. A population-based study from Rochester, Minnesota, 1976 through 1990. Brain, 1994. 117 ( Pt 2): p. 325-35.

4. Tarulli, A.W. and E.M. Raynor, Lumbosacral radiculopathy. Neurol Clin, 2007. 25(2): p. 387-405.

5. Attal, N., et al., Chronic neuropathic pain management in spinal cord injury patients. What is the efficacy of pharmacological treatments with a general mode of administration? (oral, transdermal, intravenous). Ann Phys Rehabil Med, 2009. 52(2): p. 124-41.

6. Teasell, R.W., et al., A systematic review of pharmacologic treatments of pain after spinal cord injury. Arch Phys Med Rehabil, 2010. 91(5): p. 816-31.

7. Kim, S.H. and J.M. Chung, An experimental model for peripheral neuropathy produced by segmental spinal nerve ligation in the rat. Pain, 1992. 50(3): p. 355-63.

8. Decosterd, I. and C.J. Woolf, Spared nerve injury: an animal model of persistent peripheral neuropathic pain. Pain, 2000. 87(2): p. 149-58.

9. Seltzer, Z., R. Dubner, and Y. Shir, A novel behavioral model of neuropathic pain disorders produced in rats by partial sciatic nerve injury. Pain, 1990. 43(2): p. 205-18.

10. Bennett, A.D., K.M. Chastain, and C.E. Hulsebosch, Alleviation of mechanical and thermal allodynia by CGRP(8-37) in a rodent model of chronic central pain. Pain, 2000. 86(1-2): p. 163-75.

11. Bennett, G.J. and Y.K. Xie, A peripheral mononeuropathy in rat that produces disorders of pain sensation like those seen in man. Pain, 1988. 33(1): p. 87-107.

12. Dowdall, T., I. Robinson, and T.F. Meert, Comparison of five different rat models of peripheral nerve injury. Pharmacol Biochem Behav, 2005. 80(1): p. 93-108.

13. Hu, S.J. and J.L. Xing, An experimental model for chronic compression of dorsal root ganglion produced by intervertebral foramen stenosis in the rat. Pain, 1998. 77(1): p. 15-23.

14. Li, L., et al., Effect of lumbar 5 ventral root transection on pain behaviors: a novel rat model for neuropathic pain without axotomy of primary sensory neurons. Exp Neurol, 2002. 175(1): p. 23-34.

15. Christensen, M.D., et al., Mechanical and thermal allodynia in chronic central pain following spinal cord injury. Pain, 1996. 68(1): p. 97-107.

16. Gruner, J.A., A monitored contusion model of spinal cord injury in the rat. J Neurotrauma, 1992. 9(2): p. 123-6; discussion 126-8.

17. Sandkuhler, J., Models and mechanisms of hyperalgesia and allodynia. Physiol Rev, 2009. 89(2): p. 70758.

18. Wu, S.X., et al., The synaptic connectivity that underlies the noxious transmission and modulation within the superficial dorsal horn of the spinal cord. Prog Neurobiol, 2010.

19. Cregg, R., et al., Pain Channelopathies. J Physiol, 2010. 
20. Hanani, M., Satellite glial cells in sensory ganglia: from form to function. Brain Res Brain Res Rev, 2005. 48(3): p. 457-76.

21. Amir, R. and M. Devor, Chemically mediated cross-excitation in rat dorsal root ganglia. J Neurosci, 1996. 16(15): p. 4733-41.

22. Amir, R. and M. Devor, Functional cross-excitation between afferent A- and C-neurons in dorsal root ganglia. Neuroscience, 2000. 95(1): p. 189-95.

23. Takeda, M., M. Takahashi, and S. Matsumoto, Contribution of the activation of satellite glia in sensory ganglia to pathological pain. Neurosci Biobehav Rev, 2009. 33(6): p. 784-92.

24. Ohara, P.T., et al., Gliopathic pain: when satellite glial cells go bad. Neuroscientist, 2009. 15(5): p. 450-63.

25. Novakovic, S.D., et al., Immunocytochemical localization of $P 2 X 3$ purinoceptors in sensory neurons in naive rats and following neuropathic injury. Pain, 1999. 80(1-2): p. 273-82.

26. Kage, K., et al., Alteration of dorsal root ganglion P2X3 receptor expression and function following spinal nerve ligation in the rat. Exp Brain Res, 2002. 147(4): p. 511-9.

27. Wang, R., et al., Glial cell line-derived neurotrophic factor normalizes neurochemical changes in injured dorsal root ganglion neurons and prevents the expression of experimental neuropathic pain. Neuroscience, 2003. 121(3): p. 815-24.

28. Xiang, Z., et al., Functional up-regulation of $P 2 X 3$ receptors in the chronically compressed dorsal root ganglion. Pain, 2008. 140(1): p. 23-34.

29. Honore, P., et al., Analgesic profile of intrathecal P2X(3) antisense oligonucleotide treatment in chronic inflammatory and neuropathic pain states in rats. Pain, 2002. 99(1-2): p. 11-9.

30. Barclay, J., et al., Functional downregulation of P2X3 receptor subunit in rat sensory neurons reveals a significant role in chronic neuropathic and inflammatory pain. J Neurosci, 2002. 22(18): p. 8139-47.

31. Chessell, I.P., et al., Disruption of the P2X7 purinoceptor gene abolishes chronic inflammatory and neuropathic pain. Pain, 2005. 114(3): p. 386-96.

32. Jarvis, M.F., The neural-glial purinergic receptor ensemble in chronic pain states. Trends Neurosci, 2010. 33(1): p. 48-57.

33. White, F.A., et al., Excitatory monocyte chemoattractant protein-1 signaling is up-regulated in sensory neurons after chronic compression of the dorsal root ganglion. Proc Natl Acad Sci U S A, 2005. 102(39): p. 14092-7.

34. Sun, J.H., et al., MCP-1 enhances excitability of nociceptive neurons in chronically compressed dorsal root ganglia. J Neurophysiol, 2006. 96(5): p. 2189-99.

35. Caterina, M.J., et al., Impaired nociception and pain sensation in mice lacking the capsaicin receptor. Science, 2000. 288(5464): p. 306-13.

36. Schafers, M., et al., Tumor necrosis factor-alpha induces mechanical allodynia after spinal nerve ligation by activation of p38 MAPK in primary sensory neurons. J Neurosci, 2003. 23(7): p. 2517-21.

37. Jin, S.X., et al., p38 mitogen-activated protein kinase is activated after a spinal nerve ligation in spinal cord microglia and dorsal root ganglion neurons and contributes to the generation of neuropathic pain. J Neurosci, 2003. 23(10): p. 4017-22. 
38. Xu, J.T., et al., p38 activation in uninjured primary afferent neurons and in spinal microglia contributes to the development of neuropathic pain induced by selective motor fiber injury. Exp Neurol, 2007. 204(1): p. 355-65.

39. Ramer, M.S., S.W. Thompson, and S.B. McMahon, Causes and consequences of sympathetic basket formation in dorsal root ganglia. Pain, 1999. Suppl 6: p. S111-20.

40. Jones, M.G., J.B. Munson, and S.W. Thompson, A role for nerve growth factor in sympathetic sprouting in rat dorsal root ganglia. Pain, 1999. 79(1): p. 21-9.

41. Deng, Y.S., J.H. Zhong, and X.F. Zhou, Effects of endogenous neurotrophins on sympathetic sprouting in the dorsal root ganglia and allodynia following spinal nerve injury. Exp Neurol, 2000. 164(2): p. 344-50.

42. Kingery, W.S. and J.A. Vallin, The development of chronic mechanical hyperalgesia, autotomy and collateral sprouting following sciatic nerve section in rat. Pain, 1989. 38(3): p. 321-32.

43. Diamond, J., M. Holmes, and M. Coughlin, Endogenous NGF and nerve impulses regulate the collateral sprouting of sensory axons in the skin of the adult rat. J Neurosci, 1992. 12(4): p. 1454-66.

44. Ro, L.S., et al., Effect of NGF and anti-NGF on neuropathic pain in rats following chronic constriction injury of the sciatic nerve. Pain, 1999. 79(2-3): p. 265-74.

45. Devor, M., Sodium channels and mechanisms of neuropathic pain. J Pain, 2006. 7(1 Suppl 1): p. S3-S12.

46. Devor, M., Ectopic discharge in Abeta afferents as a source of neuropathic pain. Exp Brain Res, 2009. 196(1): p. 115-28.

47. Zhang, E.T., et al., Nerve injury-induced tactile allodynia is present in the absence of FOS labeling in retrogradely labeled post-synaptic dorsal column neurons. Pain, 2007. 129(1-2): p. 143-54.

48. Hains, B.C., et al., Altered sodium channel expression in second-order spinal sensory neurons contributes to pain after peripheral nerve injury. J Neurosci, 2004. 24(20): p. 4832-9.

49. Hains, B.C. and S.G. Waxman, Activated microglia contribute to the maintenance of chronic pain after spinal cord injury. J Neurosci, 2006. 26(16): p. 4308-17.

50. Keller, A.F., et al., Transformation of the output of spinal lamina I neurons after nerve injury and microglia stimulation underlying neuropathic pain. Mol Pain, 2007. 3: p. 27.

51. Hains, B.C., W.D. Willis, and C.E. Hulsebosch, Serotonin receptors 5-HT1A and 5-HT3 reduce hyperexcitability of dorsal horn neurons after chronic spinal cord hemisection injury in rat. Exp Brain Res, 2003. 149(2): p. 174-86.

52. Neumann, S., et al., Innocuous, not noxious, input activates PKCgamma interneurons of the spinal dorsal horn via myelinated afferent fibers. J Neurosci, 2008. 28(32): p. 7936-44.

53. Miraucourt, L.S., et al., Glycine inhibitory dysfunction induces a selectively dynamic, morphine-resistant, and neurokinin 1 receptor-independent mechanical allodynia. J Neurosci, 2009. 29(8): p. 2519-27.

54. Miraucourt, L.S., R. Dallel, and D.L. Voisin, Glycine inhibitory dysfunction turns touch into pain through PKCgamma interneurons. PLoS One, 2007. 2(11): p. e1116.

55. Malmberg, A.B., et al., Preserved acute pain and reduced neuropathic pain in mice lacking PKCgamma. Science, 1997. 278(5336): p. 279-83.

56. Latremoliere, A. and C.J. Woolf, Central sensitization: a generator of pain hypersensitivity by central neural plasticity. J Pain, 2009. 10(9): p. 895-926. 
57. Scholz, J. and C.J. Woolf, The neuropathic pain triad: neurons, immune cells and glia. Nat Neurosci, 2007. 10(11): p. 1361-1368

58. Suzuki, R., L.J. Rygh, and A.H. Dickenson, Bad news from the brain: descending 5-HT pathways that control spinal pain processing. Trends Pharmacol Sci, 2004. 25(12): p. 613-7.

59. Saade, N.E. and S.J. Jabbur, Nociceptive behavior in animal models for peripheral neuropathy: spinal and supraspinal mechanisms. Prog Neurobiol, 2008. 86(1): p. 22-47.

60. Kawasaki, Y., et al., Cytokine mechanisms of central sensitization: distinct and overlapping role of interleukin-1beta, interleukin-6, and tumor necrosis factor-alpha in regulating synaptic and neuronal activity in the superficial spinal cord. J Neurosci, 2008. 28(20): p. 5189-94.

61. Qin, X., Y. Wan, and X. Wang, CCL2 and CXCL1 trigger calcitonin gene-related peptide release by exciting primary nociceptive neurons. J Neurosci Res, 2005. 82(1): p. 51-62.

62. Skoff, A.M., C. Zhao, and J.E. Adler, Interleukin-1alpha regulates substance $P$ expression and release in adult sensory neurons. Exp Neurol, 2009. 217(2): p. 395-400.

63. Reeve, A.J., et al., Intrathecally administered endotoxin or cytokines produce allodynia, hyperalgesia and changes in spinal cord neuronal responses to nociceptive stimuli in the rat. Eur J Pain, 2000. 4(3): p. 24757.

64. Milligan, E.D., et al., Evidence that exogenous and endogenous fractalkine can induce spinal nociceptive facilitation in rats. Eur J Neurosci, 2004. 20(9): p. 2294-302.

65. Kawasaki, Y., et al., Distinct roles of matrix metalloproteases in the early-and late-phase development of neuropathic pain. Nat Med, 2008. 14(3): p. 331-6.

66. Peng, X.M., et al., Tumor necrosis factor-alpha contributes to below-level neuropathic pain after spinal cord injury. Ann Neurol, 2006. 59(5): p. 843-51.

67. White, F.A., S.K. Bhangoo, and R.J. Miller, Chemokines: integrators of pain and inflammation. Nat Rev Drug Discov, 2005. 4(10): p. 834-44.

68. Abbadie, C., Chemokines, chemokine receptors and pain. Trends Immunol, 2005. 26(10): p. 529-34.

69. Zhang, J. and Y. De Koninck, Spatial and temporal relationship between monocyte chemoattractant protein-1 expression and spinal glial activation following peripheral nerve injury. J Neurochem, 2006. 97(3): p. $772-83$.

70. Abbadie, C., et al., Chemokines and pain mechanisms. Brain Res Rev, 2009. 60(1): p. 125-34.

71. Marchand, F., M. Perretti, and S.B. McMahon, Role of the immune system in chronic pain. Nat Rev Neurosci, 2005. 6(7): p. 521-32.

72. Okamoto, K., et al., Pro- and anti-inflammatory cytokine gene expression in rat sciatic nerve chronic constriction injury model of neuropathic pain. Exp Neurol, 2001. 169(2): p. 386-91.

73. Mika, J., et al., Interleukin-1 alpha has antiallodynic and antihyperalgesic activities in a rat neuropathic pain model. Pain, 2008. 138(3): p. 587-97.

74. Rothman, S.M., et al., Cytokine mRNA expression in painful radiculopathy. J Pain, 2009. 10(1): p. 90-9.

75. Jeon, S.M., K.M. Lee, and H.J. Cho, Expression of monocyte chemoattractant protein-1 in rat dorsal root ganglia and spinal cord in experimental models of neuropathic pain. Brain Res, 2009. 1251: p. 103-11.

76. Streit, W.J., et al., Cytokine mRNA profiles in contused spinal cord and axotomized facial nucleus suggest a beneficial role for inflammation and gliosis. Exp Neurol, 1998. 152(1): p. 74-87. 
77. Donnelly, D.J. and P.G. Popovich, Inflammation and its role in neuroprotection, axonal regeneration and functional recovery after spinal cord injury. Exp Neurol, 2007.

78. Hains, B.C., J.A. Yucra, and C.E. Hulsebosch, Reduction of pathological and behavioral deficits following spinal cord contusion injury with the selective cyclooxygenase-2 inhibitor NS-398. J Neurotrauma, 2001. 18(4): p. 409-23.

79. Zhao, P., S.G. Waxman, and B.C. Hains, Extracellular signal-regulated kinase-regulated microglia-neuron signaling by prostaglandin E2 contributes to pain after spinal cord injury. J Neurosci, 2007. 27(9): p. 235768.

80. Watkins, L.R. and S.F. Maier, Beyond neurons: evidence that immune and glial cells contribute to pathological pain states. Physiol Rev, 2002. 82(4): p. 981-1011.

81. Zhang, J., et al., Expression of CCR2 in both resident and bone marrow-derived microglia plays a critical role in neuropathic pain. J Neurosci, 2007. 27(45): p. 12396-406.

82. Cao, L. and J.A. DeLeo, CNS-infiltrating CD4+ Tlymphocytes contribute to murine spinal nerve transectioninduced neuropathic pain. Eur J Immunol, 2008. 38(2): p. 448-58.

83. De Leo, J.A., V.L. Tawfik, and M.L. LaCroix-Fralish, The tetrapartite synapse: path to CNS sensitization and chronic pain. Pain, 2006. 122(1-2): p. 17-21.

84. Haydon, P.G. and G. Carmignoto, Astrocyte control of synaptic transmission and neurovascular coupling. Physiol Rev, 2006. 86(3): p. 1009-31.

85. Perea, G., M. Navarrete, and A. Araque, Tripartite synapses: astrocytes process and control synaptic information. Trends Neurosci, 2009. 32(8): p. 421-31.

86. Davalos, D., et al., ATP mediates rapid microglial response to local brain injury in vivo. Nat Neurosci, 2005. 8(6): p. $752-8$.

87. Nimmerjahn, A., F. Kirchhoff, and F. Helmchen, Resting microglial cells are highly dynamic surveillants of brain parenchyma in vivo. Science, 2005. 308(5726): p. 1314-8.

88. Eng, L.F., R.S. Ghirnikar, and Y.L. Lee, Glial fibrillary acidic protein: GFAP-thirty-one years (1969-2000). Neurochem Res, 2000. 25(9-10): p. 1439-51.

89. Ling, E.A., et al., Immunocytochemical localization of CR3 complement receptors with OX-42 in amoeboid microglia in postnatal rats. Anat Embryol (Berl), 1990. 182(5): p. 481-6.

90. Ito, D., et al., Microglia-specific localisation of a novel calcium binding protein, Iba1. Brain Res Mol Brain Res, 1998. 57(1): p. 1-9.

91. Romero-Sandoval, A., et al., A comparison of spinal Ibal and GFAP expression in rodent models of acute and chronic pain. Brain Res, 2008. 1219: p. 116-26.

92. Coyle, D.E., Partial peripheral nerve injury leads to activation of astroglia and microglia which parallels the development of allodynic behavior. Glia, 1998. 23(1): p. 75-83.

93. Hu, P., et al., Immune cell involvement in dorsal root ganglia and spinal cord after chronic constriction or transection of the rat sciatic nerve. Brain Behav Immun, 2007. 21(5): p. 599-616.

94. Ransohoff, R.M. and V.H. Perry, Microglial physiology: unique stimuli, specialized responses. Annu Rev Immunol, 2009. 27: p. 119-45.

95. McMahon, S.B. and M. Malcangio, Current challenges in glia-pain biology. Neuron, 2009. 64(1): p. 4654. 
96. Tsuda, M., K. Inoue, and M.W. Salter, Neuropathic pain and spinal microglia: a big problem from molecules in "small" glia. Trends Neurosci, 2005. 28(2): p. 101-7.

97. Coull, J.A., et al., BDNF from microglia causes the shift in neuronal anion gradient underlying neuropathic pain. Nature, 2005. 438(7070): p. 1017-21.

98. Coull, J.A., et al., Trans-synaptic shift in anion gradient in spinal lamina I neurons as a mechanism of neuropathic pain. Nature, 2003. 424(6951): p. 938-42.

99. Broom, D.C., et al., Cyclooxygenase 2 expression in the spared nerve injury model of neuropathic pain. Neuroscience, 2004. 124(4): p. 891-900.

100. Rothstein, J.D., et al., Knockout of glutamate transporters reveals a major role for astroglial transport in excitotoxicity and clearance of glutamate. Neuron, 1996. 16(3): p. 675-86.

101. Sung, B., G. Lim, and J. Mao, Altered expression and uptake activity of spinal glutamate transporters after nerve injury contribute to the pathogenesis of neuropathic pain in rats. J Neurosci, 2003. 23(7): p. 2899910.

102. Tawfik, V.L., et al., Propentofylline-induced astrocyte modulation leads to alterations in glial glutamate promoter activation following spinal nerve transection. Neuroscience, 2008. 152(4): p. 1086-92.

103. Kozai, T., et al., Tissue type plasminogen activator induced in rat dorsal horn astrocytes contributes to mechanical hypersensitivity following dorsal root injury. Glia, 2007. 55(6): p. 595-603.

104. Zhuang, Z.Y., et al., A peptide c-Jun N-terminal kinase (JNK) inhibitor blocks mechanical allodynia after spinal nerve ligation: respective roles of JNK activation in primary sensory neurons and spinal astrocytes for neuropathic pain development and maintenance. J Neurosci, 2006. 26(13): p. 3551-60.

105. Gao, Y.J., et al., JNK-induced MCP-1 production in spinal cord astrocytes contributes to central sensitization and neuropathic pain. J Neurosci, 2009. 29(13): p. 4096-108.

106. Deumens, R., et al., Inverse relation between intensity of GFAP expression in the substantia gelatinosa and degree of chronic mechanical allodynia. Neurosci Lett, 2009. 452(2): p. 101-5.

107. Porreca, F., M.H. Ossipov, and G.F. Gebhart, Chronic pain and medullary descending facilitation. Trends Neurosci, 2002. 25(6): p. 319-25.

108. Zhao, P., S.G. Waxman, and B.C. Hains, Modulation of thalamic nociceptive processing after spinal cord injury through remote activation of thalamic microglia by cysteine cysteine chemokine ligand 21. J Neurosci, 2007. 27(33): p. 8893-902.

109. Nassar, M.A., et al., Nociceptor-specific gene deletion reveals a major role for Nav1.7 (PN1) in acute and inflammatory pain. Proc Natl Acad Sci U S A, 2004. 101(34): p. 12706-11.

110. Priest, B.T., et al., Contribution of the tetrodotoxin-resistant voltage-gated sodium channel NaV1.9 to sensory transmission and nociceptive behavior. Proc Natl Acad Sci U S A, 2005. 102(26): p. 9382-7.

111. Nassar, M.A., et al., Nerve injury induces robust allodynia and ectopic discharges in Nav1.3 null mutant mice. Mol Pain, 2006. 2: p. 33.

112. Zimmermann, K., et al., Sensory neuron sodium channel Nav1.8 is essential for pain at low temperatures. Nature, 2007. 447(7146): p. 855-8.

113. Abrahamsen, B., et al., The cell and molecular basis of mechanical, cold, and inflammatory pain. Science, 2008. 321(5889): p. 702-5. 
114. Sukhotinsky, I., et al., Key role of the dorsal root ganglion in neuropathic tactile hypersensibility. Eur J Pain, 2004. 8(2): p. 135-43.

115. Woolf, C.J., P. Shortland, and R.E. Coggeshall, Peripheral nerve injury triggers central sprouting of myelinated afferents. Nature, 1992. 355(6355): p. 75-8.

116. Woolf, C.J., et al., Reorganization of central terminals of myelinated primary afferents in the rat dorsal horn following peripheral axotomy. J Comp Neurol, 1995. 360(1): p. 121-34.

117. Lekan, H.A., S.M. Carlton, and R.E. Coggeshall, Sprouting of A beta fibers into lamina II of the rat dorsal horn in peripheral neuropathy. Neurosci Lett, 1996. 208(3): p. 147-50.

118. Doubell, T.P., R.J. Mannion, and C.J. Woolf, Intact sciatic myelinated primary afferent terminals collaterally sprout in the adult rat dorsal horn following section of a neighbouring peripheral nerve. J Comp Neurol, 1997. 380(1): p. 95-104.

119. Shortland, P., E. Kinman, and C. Molander, Sprouting of A-fibre primary afferents into lamina II in two rat models of neuropathic pain. Eur J Pain, 1997. 1(3): p. 215-27.

120. Nakamura, S. and R.R. Myers, Myelinated afferents sprout into lamina II of L3-5 dorsal horn following chronic constriction nerve injury in rats. Brain Res, 1999. 818(2): p. 285-90.

121. Bao, L., et al., Peripheral axotomy induces only very limited sprouting of coarse myelinated afferents into inner lamina Il of rat spinal cord. Eur J Neurosci, 2002. 16(2): p. 175-85.

122. Hughes, D.I., et al., Lack of evidence for sprouting of Abeta afferents into the superficial laminas of the spinal cord dorsal horn after nerve section. J Neurosci, 2003. 23(29): p. 9491-9.

123. Doubell, T.P. and C.J. Woolf, Growth-associated protein 43 immunoreactivity in the superficial dorsal horn of the rat spinal cord is localized in atrophic C-fiber, and not in sprouted A-fiber, central terminals after peripheral nerve injury. J Comp Neurol, 1997. 386(1): p. 111-8.

124. Hu, J., et al., Central sprouting of uninjured small fiber afferents in the adult rat spinal cord following spinal nerve ligation. Eur J Neurosci, 2004. 20(7): p. 1705-12.

125. Christensen, M.D. and C.E. Hulsebosch, Spinal cord injury and anti-NGF treatment results in changes in CGRP density and distribution in the dorsal horn in the rat. Exp Neurol, 1997. 147(2): p. 463-75.

126. Deumens, R., et al., Mice lacking $L 1$ have reduced CGRP fibre in-growth into spinal transection lesions. Neurosci Lett, 2007. 420(3): p. 277-281.

127. Lu, B., P.T. Pang, and N.H. Woo, The yin and yang of neurotrophin action. Nat Rev Neurosci, 2005. 6(8): p. $603-14$.

128. Hunt, S.P. and P.W. Mantyh, The molecular dynamics of pain control. Nat Rev Neurosci, 2001. 2(2): p. 83-91.

129. Deumens, R., G.C. Koopmans, and E.A. Joosten, Regeneration of descending axon tracts after spinal cord injury. Prog Neurobiol, 2005. 77(1-2): p. 57-89.

130. Krenz, N.R. and L.C. Weaver, Nerve growth factor in glia and inflammatory cells of the injured rat spinal cord. J Neurochem, 2000. 74(2): p. 730-9.

131. Brown, A., M.J. Ricci, and L.C. Weaver, NGF message and protein distribution in the injured rat spinal cord. Exp Neurol, 2004. 188(1): p. 115-27.

132. Tang, X.Q., D.L. Tanelian, and G.M. Smith, Semaphorin3A inhibits nerve growth factor-induced sprouting of nociceptive afferents in adult rat spinal cord. J Neurosci, 2004. 24(4): p. 819-27. 
133. Jaken, R.J., et al., Synaptic plasticity in the substantia gelatinosa in a model of chronic neuropathic pain. Neurosci Lett, 2010. 469(1): p. 30-3.

134. Marchand, J.E., et al., Altered tachykinin expression by dorsal root ganglion neurons in a rat model of neuropathic pain. Pain, 1994. 58(2): p. 219-31.

135. Ma, W., M.S. Ramer, and M.A. Bisby, Increased calcitonin gene-related peptide immunoreactivity in gracile nucleus after partial sciatic nerve injury: age-dependent and originating from spared sensory neurons. Exp Neurol, 1999. 159(2): p. 459-73.

136. Michael, G.J., et al., Axotomy results in major changes in BDNF expression by dorsal root ganglion cells: BDNF expression in large trkB and trkC cells, in pericellular baskets, and in projections to deep dorsal horn and dorsal column nuclei. Eur J Neurosci, 1999. 11(10): p. 3539-51.

137. Malcangio, M., et al., Abnormal substance P release from the spinal cord following injury to primary sensory neurons. Eur J Neurosci, 2000. 12(1): p. 397-9.

138. Weissner, W., et al., Time course of substance P expression in dorsal root ganglia following complete spinal nerve transection. J Comp Neurol, 2006. 497(1): p. 78-87.

139. Moore, K.A., et al., Partial peripheral nerve injury promotes a selective loss of GABAergic inhibition in the superficial dorsal horn of the spinal cord. J Neurosci, 2002. 22(15): p. 6724-31.

140. Scholz, J., et al., Blocking caspase activity prevents transsynaptic neuronal apoptosis and the loss of inhibition in lamina ll of the dorsal horn after peripheral nerve injury. J Neurosci, 2005. 25(32): p. 7317-23.

141. Lever, I., et al., Release of BDNF and GABA in the dorsal horn of neuropathic rats. Eur J Neurosci, 2003. 18(5): p. 1169-74.

142. Stiller, C.O., et al., Release of gamma-aminobutyric acid in the dorsal horn and suppression of tactile allodynia by spinal cord stimulation in mononeuropathic rats. Neurosurgery, 1996. 39(2): p. 367-74; discussion 374-5.

143. Engle, M.P., et al., Spinal nerve ligation does not alter the expression or function of $G A B A(B)$ receptors in spinal cord and dorsal root ganglia of the rat. Neuroscience, 2006. 138(4): p. 1277-87.

144. Polgar, E. and A.J. Todd, Tactile allodynia can occur in the spared nerve injury model in the rat without selective loss of GABA or GABA(A) receptors from synapses in laminael-Il of the ipsilateral spinal dorsal horn. Neuroscience, 2008. 156(1): p. 193-202.

145. Schoffnegger, D., et al., Physiological properties of spinal lamina /l GABAergic neurons in mice following peripheral nerve injury. J Physiol, 2006. 577(Pt 3): p. 869-78.

146. Meisner, J.G., A.D. Marsh, and D.R. Marsh, Loss of GABAergic interneurons in laminae I-III of the spinal cord dorsal horn contributes to reduced GABAergic tone and neuropathic pain after spinal cord injury. J Neurotrauma, 2010. 27(4): p. 729-37.

147. Schmidli, R.S., et al., Cytokine regulation of glutamate decarboxylase biosynthesis in isolated rat islets of Langerhans. Biochem J, 1996. 317 ( Pt 3): p. 713-9.

148. Polgar, E., et al., Selective loss of spinal GABAergic or glycinergic neurons is not necessary for development of thermal hyperalgesia in the chronic constriction injury model of neuropathic pain. Pain, 2003. 104(1-2): p. 229-39. 
149. Polgar, E., et al., Loss of neurons from laminas I-III of the spinal dorsal horn is not required for development of tactile allodynia in the spared nerve injury model of neuropathic pain. J Neurosci, 2005. 25(28): p. 6658-66.

150. Streit, W.J., S.A. Walter, and N.A. Pennell, Reactive microgliosis. Prog Neurobiol, 1999. 57(6): p. 563-81.

151. Tanga, F.Y., V. Raghavendra, and J.A. DeLeo, Quantitative real-time RT-PCR assessment of spinal microglial and astrocytic activation markers in a rat model of neuropathic pain. Neurochem Int, 2004. 45(2-3): p. 397-407.

152. Kigerl, K.A., et al., Toll-like receptor (TLR)-2 and TLR-4 regulate inflammation, gliosis, and myelin sparing after spinal cord injury. J Neurochem, 2007. 102(1): p. 37-50.

153. Tanga, F.Y., N. Nutile-McMenemy, and J.A. DeLeo, The CNS role of Toll-like receptor 4 in innate neuroimmunity and painful neuropathy. Proc Natl Acad Sci U S A, 2005. 102(16): p. 5856-61.

154. Kim, D., et al., A critical role of toll-like receptor 2 in nerve injury-induced spinal cord glial cell activation and pain hypersensitivity. J Biol Chem, 2007. 282(20): p. 14975-83.

155. Obata, K., et al., Toll-like receptor 3 contributes to spinal glial activation and tactile allodynia after nerve injury. J Neurochem, 2008.

156. Hutchinson, M.R., et al., Evidence for a role of heat shock protein-90 in toll like receptor 4 mediated pain enhancement in rats. Neuroscience, 2009. 164(4): p. 1821-32.

157. Krueger-Naug, A.M., et al., Hyperthermic induction of the 27-kDa heat shock protein (Hsp27) in neuroglia and neurons of the rat central nervous system. J Comp Neurol, 2000. 428(3): p. 495-510.

158. Benn, S.C., et al., Hsp27 upregulation and phosphorylation is required for injured sensory and motor neuron survival. Neuron, 2002. 36(1): p. 45-56.

159. Kigerl, K.A. and P.G. Popovich, Toll-like receptors in spinal cord injury. Curr Top Microbiol Immunol, 2009 336: p. 121-36.

160. Honda, S., et al., Extracellular ATP or ADP induce chemotaxis of cultured microglia through Gi/o-coupled P2Y receptors. J Neurosci, 2001. 21(6): p. 1975-82.

161. Haynes, S.E., et al., The P2Y12 receptor regulates microglial activation by extracellular nucleotides. Nat Neurosci, 2006. 9(12): p. 1512-9.

162. Wu, L.J., K.I. Vadakkan, and M. Zhuo, ATP-induced chemotaxis of microglial processes requires $P 2 Y$ receptor-activated initiation of outward potassium currents. Glia, 2007. 55(8): p. 810-21.

163. Kobayashi, K., et al., P2Y12 receptor upregulation in activated microglia is a gateway of p38 signaling and neuropathic pain. J Neurosci, 2008. 28(11): p. 2892-902.

164. Tozaki-Saitoh, H., et al., P2Y12 receptors in spinal microglia are required for neuropathic pain after peripheral nerve injury. J Neurosci, 2008. 28(19): p. 4949-56.

165. Kawate, T., et al., Crystal structure of the ATP-gated P2X(4) ion channel in the closed state. Nature, 2009. 460(7255): p. 592-8.

166. Tsuda, M., et al., P2X4 receptors induced in spinal microglia gate tactile allodynia after nerve injury. Nature, 2003. 424(6950): p. 778-83.

167. Ulmann, L., et al., Up-regulation of P2X4 receptors in spinal microglia after peripheral nerve injury mediates BDNF release and neuropathic pain. J Neurosci, 2008. 28(44): p. 11263-8. 
168. Trang, T., et al., P2X4-receptor-mediated synthesis and release of brain-derived neurotrophic factor in microglia is dependent on calcium and p38-mitogen-activated protein kinase activation. J Neurosci, 2009. 29(11): p. 3518-28.

169. Tsuda, M., et al., Activation of $p 38$ mitogen-activated protein kinase in spinal hyperactive microglia contributes to pain hypersensitivity following peripheral nerve injury. Glia, 2004. 45(1): p. 89-95.

170. Tsuda, M., et al., Lyn tyrosine kinase is required for $P 2 X(4)$ receptor upregulation and neuropathic pain after peripheral nerve injury. Glia, 2008. 56(1): p. 50-8.

171. Tsuda, M., et al., Fibronectin/integrin system is involved in P2X(4) receptor upregulation in the spinal cord and neuropathic pain after nerve injury. Glia, 2008. 56(5): p. 579-85.

172. Nasu-Tada, K., et al., Possible involvement of increase in spinal fibronectin following peripheral nerve injury in upregulation of microglial P2X4, a key molecule for mechanical allodynia. Glia, 2006. 53(7): p. 769-75.

173. Gordh, T., H. Chu, and H.S. Sharma, Spinal nerve lesion alters blood-spinal cord barrier function and activates astrocytes in the rat. Pain, 2006. 124(1-2): p. 211-21.

174. Dansereau, M.A., et al., Spinal CCL2 pronociceptive action is no longer effective in CCR2 receptor antagonist-treated rats. J Neurochem, 2008. 106(2): p. 757-69.

175. Thacker, M.A., et al., CCL2 is a key mediator of microglia activation in neuropathic pain states. Eur J Pain, 2009. 13(3): p. 263-72.

176. Abbadie, C., et al., Impaired neuropathic pain responses in mice lacking the chemokine receptor CCR2. Proc Natl Acad Sci U S A, 2003. 100(13): p. 7947-52.

177. Clark, A.K., P.K. Yip, and M. Malcangio, The liberation of fractalkine in the dorsal horn requires microglial cathepsin S. J Neurosci, 2009. 29(21): p. 6945-54.

178. Verge, G.M., et al., Fractalkine (CX3CL1) and fractalkine receptor (CX3CR1) distribution in spinal cord and dorsal root ganglia under basal and neuropathic pain conditions. Eur J Neurosci, 2004. 20(5): p. 115060.

179. Wen, Y.R., et al., Nerve conduction blockade in the sciatic nerve prevents but does not reverse the activation of 38 mitogen-activated protein kinase in spinal microglia in the rat spared nerve injury model. Anesthesiology, 2007. 107(2): p. 312-21.

180. Suter, M.R., et al., Large A-fiber activity is required for microglial proliferation and p38 MAPK activation in the spinal cord: different effects of resiniferatoxin and bupivacaine on spinal microglial changes after spared nerve injury. Mol Pain, 2009. 5: p. 53.

181. Tilleux, S., J. Berger, and E. Hermans, Induction of astrogliosis by activated microglia is associated with a down-regulation of metabotropic glutamate receptor 5. J Neuroimmunol, 2007. 189(1-2): p. 23-30.

182. Tilleux, S. and E. Hermans, Down-regulation of astrocytic GLAST by microglia-related inflammation is abrogated in dibutyryl cAMP-differentiated cultures. J Neurochem, 2008.

183. Miyoshi, K., et al., Interleukin-18-mediated microglia/astrocyte interaction in the spinal cord enhances neuropathic pain processing after nerve injury. J Neurosci, 2008. 28(48): p. 12775-87.

184. Colburn, R.W., A.J. Rickman, and J.A. DeLeo, The effect of site and type of nerve injury on spinal glial activation and neuropathic pain behavior. Exp Neurol, 1999. 157(2): p. 289-304.

185. Scholz, J., et al., Low-dose methotrexate reduces peripheral nerve injury-evoked spinal microglial activation and neuropathic pain behavior in rats. Pain, 2008. 138(1): p. 130-42. 
186. Wang, W., et al., Crosstalk between spinal astrocytes and neurons in nerve injury-induced neuropathic pain. PLoS One, 2009. 4(9): p. e6973.

187. Garrison, C.J., P.M. Dougherty, and S.M. Carlton, GFAP expression in lumbar spinal cord of naive and neuropathic rats treated with MK-801. Exp Neurol, 1994. 129(2): p. 237-43.

188. Klusakova, I. and P. Dubovy, Experimental models of peripheral neuropathic pain based on traumatic nerve injuries - an anatomical perspective. Ann Anat, 2009. 191(3): p. 248-59.

189. Eaton, M.J., et al., Transplants of neuronal cells bioengineered to synthesize GABA alleviate chronic neuropathic pain. Cell Transplant, 1999. 8(1): p. 87-101.

190. Eaton, M.J., et al., Initial characterization of the transplant of immortalized chromaffin cells for the attenuation of chronic neuropathic pain. Cell Transplant, 2000. 9(5): p. 637-56.

191. Hains, B.C., et al., Engraftment of serotonergic precursors enhances locomotor function and attenuates chronic central pain behavior following spinal hemisection injury in the rat. Exp Neurol, 2001. 171(2): p. $361-78$.

192. Hains, B.C., et al., Serotonergic neural precursor cell grafts attenuate bilateral hyperexcitability of dorsal horn neurons after spinal hemisection in rat. Neuroscience, 2003. 116(4): p. 1097-110.

193. Stubley, L.A., et al., Only early intervention with gamma-aminobutyric acid cell therapy is able to reverse neuropathic pain after partial nerve injury. J Neurotrauma, 2001. 18(4): p. 471-7.

194. Eaton, M.J., et al., Subarachnoid transplant of a human neuronal cell line attenuates chronic allodynia and hyperalgesia after excitotoxic spinal cord injury in the rat. J Pain, 2007. 8(1): p. 33-50.

195. Eaton, M.J. and S.Q. Wolfe, Clinical feasibility for cell therapy using human neuronal cell line to treat neuropathic behavioral hypersensitivity following spinal cord injury in rats. J Rehabil Res Dev, 2009. 46(1): p. 145-65.

196. Hofstetter, C.P., et al., Allodynia limits the usefulness of intraspinal neural stem cell grafts; directed differentiation improves outcome. Nat Neurosci, 2005. 8(3): p. 346-53.

197. Deumens, R., et al., Locomotor dysfunction and pain: the scylla and charybdis of fiber sprouting after spinal cord injury. Mol Neurobiol, 2008. 37(1): p. 52-63.

198. Li, Y., P.M. Field, and G. Raisman, Repair of adult rat corticospinal tract by transplants of olfactory ensheathing cells. Science, 1997. 277(5334): p. 2000-2.

199. Ramon-Cueto, A., et al., Functional recovery of paraplegic rats and motor axon regeneration in their spinal cords by olfactory ensheathing glia. Neuron, 2000. 25(2): p. 425-35.

200. Deumens, R., et al., Olfactory ensheathing cells, olfactory nerve fibroblasts and biomatrices to promote long-distance axon regrowth and functional recovery in the dorsally hemisected adult rat spinal cord. Exp Neurol, 2006. 200(1): p. 89-103.

201. Lakatos, A., R.J. Franklin, and S.C. Barnett, Olfactory ensheathing cells and Schwann cells differ in their in vitro interactions with astrocytes. Glia, 2000. 32(3): p. 214-25.

202. Dombrowski, M.A., et al., Myelination and nodal formation of regenerated peripheral nerve fibers following transplantation of acutely prepared olfactory ensheathing cells. Brain Res, 2006. 1125(1): p. 1-8.

203. Radtke, C., et al., Transplantation of olfactory ensheathing cells enhances peripheral nerve regeneration after microsurgical nerve repair. Brain Res, 2009. 1254: p. 10-7. 
204. Mackay-Sim, A., et al., Autologous olfactory ensheathing cell transplantation in human paraplegia: a 3-year clinical trial. Brain, 2008. 131(Pt 9): p. 2376-86.

205. Gao, Y.J. and R.R. Ji, Chemokines, neuronal-glial interactions, and central processing of neuropathic pain. Pharmacol Ther, 2010. 126(1): p. 56-68.

206. Capelle, H.H., et al., Neurotrophins in the cerebrospinal fluid of patient cohorts with neuropathic pain, nociceptive pain, or normal pressure hydrocephalus. Clin J Pain, 2009. 25(8): p. 729-33.

207. Boucher, T.J., et al., Potent analgesic effects of GDNF in neuropathic pain states. Science, 2000. 290(5489): p. 124-7.

208. Wall, P.D. and M. Devor, Sensory afferent impulses originate from dorsal root ganglia as well as from the periphery in normal and nerve injured rats. Pain, 1983. 17(4): p. 321-39.

209. Study, R.E. and M.G. Kral, Spontaneous action potential activity in isolated dorsal root ganglion neurons from rats with a painful neuropathy. Pain, 1996. 65(2-3): p. 235-42.

210. Zhao, P., S.G. Waxman, and B.C. Hains, Sodium channel expression in the ventral posterolateral nucleus of the thalamus after peripheral nerve injury. Mol Pain, 2006. 2: p. 27.

211. Hains, B.C., et al., Upregulation of sodium channel Nav1.3 and functional involvement in neuronal hyperexcitability associated with central neuropathic pain after spinal cord injury. J Neurosci, 2003. 23(26): p. 8881-92.

212. Hains, B.C., C.Y. Saab, and S.G. Waxman, Changes in electrophysiological properties and sodium channel Nav1.3 expression in thalamic neurons after spinal cord injury. Brain, 2005. 128(Pt 10): p. 2359-71.

213. Carlton, S.M., et al., Peripheral and central sensitization in remote spinal cord regions contribute to central neuropathic pain after spinal cord injury. Pain, 2009. 147(1-3): p. 265-76.

214. Kuzumaki, N., et al., Chronic pain-induced astrocyte activation in the cingulate cortex with no change in neural or glial differentiation from neural stem cells in mice. Neurosci Lett, 2007. 415(1): p. 22-7.

215. Oosterhof, J., et al., Predicting outcome of TENS in chronic pain: a prospective, randomized, placebo controlled trial. Pain, 2008. 136(1-2): p. 11-20.

216. Norrbrink, C., Transcutaneous electrical nerve stimulation for treatment of spinal cord injury neuropathic pain. J Rehabil Res Dev, 2009. 46(1): p. 85-93.

217. Van Zundert, J., et al., Pulsed radiofrequency adjacent to the cervical dorsal root ganglion in chronic cervical radicular pain: a double blind sham controlled randomized clinical trial. Pain, 2007. 127(1-2): p. 17382.

218. Kemler, M.A., et al., Spinal cord stimulation in patients with chronic reflex sympathetic dystrophy. N Engl J Med, 2000. 343(9): p. 618-24.

219. Cruccu, G., et al., EFNS guidelines on neurostimulation therapy for neuropathic pain. Eur J Neurol, 2007. 14(9): p. 952-70.

220. Tesfaye, S., et al., Electrical spinal-cord stimulation for painful diabetic peripheral neuropathy. Lancet, 1996. 348(9043): p. 1698-701.

221. Daousi, C., S.J. Benbow, and I.A. MacFarlane, Electrical spinal cord stimulation in the long-term treatment of chronic painful diabetic neuropathy. Diabet Med, 2005. 22(4): p. 393-8.

222. Kemler, M.A., et al., Effect of spinal cord stimulation for chronic complex regional pain syndrome Type l: five-year final follow-up of patients in a randomized controlled trial. J Neurosurg, 2008. 108(2): p. 292-8. 
223. Smits, H., et al., Effect of spinal cord stimulation in an animal model of neuropathic pain relates to degree of tactile "allodynia". Neuroscience, 2006. 143(2): p. 541-6.

224. van Eijs, F., et al., Brush-evoked allodynia predicts outcome of spinal cord stimulation in complex regional pain syndrome type 1. Eur J Pain. 14(2): p. 164-9.

225. Smits, H., et al., Spinal cord stimulation induces c-Fos expression in the dorsal horn in rats with neuropathic pain after partial sciatic nerve injury. Neurosci Lett, 2009. 450(1): p. 70-3.

226. Cui, J.G., et al., Spinal cord stimulation attenuates augmented dorsal horn release of excitatory amino acids in mononeuropathy via a GABAergic mechanism. Pain, 1997. 73(1): p. 87-95.

227. Cui, J.G., B. Linderoth, and B.A. Meyerson, Effects of spinal cord stimulation on touch-evoked allodynia involve GABAergic mechanisms. An experimental study in the mononeuropathic rat. Pain, 1996. 66(2-3): p. 287-95.

228. Lind, G., et al., Intrathecal baclofen as adjuvant therapy to enhance the effect of spinal cord stimulation in neuropathic pain: a pilot study. Eur J Pain, 2004. 8(4): p. 377-83.

229. Lind, G., et al., Baclofen-enhanced spinal cord stimulation and intrathecal baclofen alone for neuropathic pain: Long-term outcome of a pilot study. Eur J Pain, 2008. 12(1): p. 132-6.

230. Munglani, R., The longer term effect of pulsed radiofrequency for neuropathic pain. Pain, 1999. 80(1-2): p. 437-9.

231. Sluijter, M.E., The role of radiofrequency in failed back surgery patients. Curr Rev Pain, 2000. 4(1): p. 4953.

232. Protasoni, M., et al., Pulsed radiofrequency effects on the lumbar ganglion of the rat dorsal root: a morphological light and transmission electron microscopy study at acute stage. Eur Spine J, 2009. 18(4): p. 473-8.

233. Cahana, A., L. Vutskits, and D. Muller, Acute differential modulation of synaptic transmission and cell survival during exposure to pulsed and continuous radiofrequency energy. J Pain, 2003. 4(4): p. 197-202.

234. Van Zundert, J., et al., Pulsed and continuous radiofrequency current adjacent to the cervical dorsal root ganglion of the rat induces late cellular activity in the dorsal horn. Anesthesiology, 2005. 102(1): p. 12531.

235. Ozsoylar, O., et al., Percutaneous pulsed radiofrequency reduce mechanical allodynia in a neuropathic pain model. Anesth Analg, 2008. 107(4): p. 1406-11. 



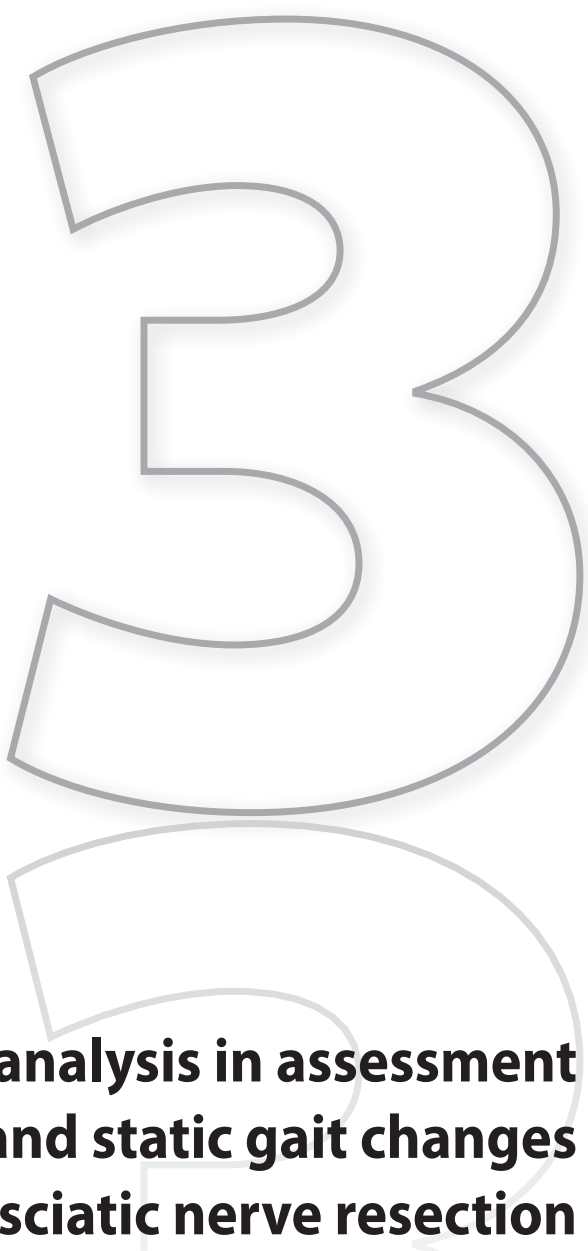

R. Deumens, R.J.P. Jaken, M.A.E. Marcus, E.A.J. Joosten 


\section{Abstract}

Functional repair of neurotmesis has been proven most challenging in regenerative medicine. Progress in this field has shown that functional repair not only requires axon regeneration, but also selectivity in target reinnervation. Although selectivity in target reinnervation still involves relatively unexplored avenues, evidencebased medicine, in the end, requires behavioral proof of repair. Therefore, there is a need for tests assessing behavioral deficits after neurotmesis. To date, behavioral tests for detecting both dynamic and static parameters are limited. The CatWalk gait analysis has been shown to detect a multitude of speed-controlled dynamic and static gait deficits after experimental spinal cord injury. Therefore, we here evaluated its use in detecting both dynamic and static gait deficits after neurotmesis. After rat sciatic nerve resection CatWalk testing was performed for eight weeks. A large amount of dynamic and static gait parameters were detected to be immediately and severely affected in the ipsilateral paw, sometimes reaching levels of only $15 \%$ of those of the unaffected paw. We conclude that the CatWalk objectively detects dynamic and static gait impairments after sciatic nerve resection and future experiments are now required to prove which of these parameters are of particular interest to detect functional repair. 


\section{Introduction}

The prevalence of peripheral nerve injuries (PNI) is estimated at a $2.8 \%$ of all trauma patients [1]. Although most of these injuries are not life threatening, they have a huge socio-economic impact [2]. PNI may manifest in motor, sensory, and autonomic dysfunctions, depending on the nerve affected. The most challenging PNI with respect to repair is represented by complete nerve transection (neurotmesis), because the intrinsic regeneration capacity of injured peripheral axons is strongly impeded by disruption of endoneurial tubes in which these axons lie. Surgical coaptation of nerve stumps is not always possible or satisfactory, especially not for PNI with large lesion gaps [3]. Therefore, a large body of research nowadays focuses on the development of synthetic nerve guides and the stimulation of peripheral axon regeneration across large PNI gaps [4]. Such guides may serve as alternatives for autologous nerve transplantation, which is routinely used in the clinic, but has negative side-effects including co-morbidity and limited availability of donor tissue.

The behavioral benefit of synthetic bridges may represent the most important factor determining its use as a clinically relevant tool to repair neurotmesis. The most frequently used behavioral test for evaluation of neurotmesis-induced deficits is the walking track analysis from which the sciatic function index (SFI) has been calculated $[5,6]$. Calculation of the SFI involves measurements of paw print length and toe spreading and therefore requires clear prints of the toes of the paws. However, such prints are strongly hampered by neurotmesis-induced phenomena like flexion contractures, autotomy, smearing of the print and dragging of the tail [7], thereby limiting the use of the SFI. Moreover, speed of gait, which is difficult to control using the walking track, is a confounder to many gait parameters [8]. Additionally, neurotmesis not only induces changes in static but also dynamic gait parameters. Therefore, additional tests have been developed including gait-stance duration and kinematic ankle measurements [8-10]. Although each of these tests assesses aspects of behavioral deficit after neurotmesis, there is still no speed-controlled behavioral test detecting a wide range of both dynamic and static gait alterations after neurotmesis. After experimental spinal cord injury, a multitude of affected dynamic and static gait parameters have been detected using the CatWalk gait analysis [11-13], a behavioral test in which speed of gait can also be fairly easily be controlled. This objective and automated gait analysis may, therefore, also be used to detect gait alterations after neurotmesis. The goal of the present study was to evaluate the magnitude and extent of dynamic and static gait changes after adult rat sciatic nerve resection.

\section{Materials and methods}

\section{Animals}

All experimental procedures were performed according to the recommendations of the European Commission (European Communities Council Directive of 24 November 1986; 86/609/EEC) and protocols were approved by the Committee on Animal Research of the Maastricht University 
(DEC 2006-054). In this study, every attempt was made to minimize the number of animals and their pain and discomfort. A total of 36 male Wistar rats (Charles River, The Netherlands) aged seven weeks, were used in this study. Throughout the study, these animals were individually housed in a temperature $\left(19-24^{\circ} \mathrm{C}\right)$ and humidity $(55 \pm 15 \%)$ conditioned room with a normal 12:12 h light/dark regime and background music to reduce stress. Throughout the preoperative behavioral training period (see below) and after surgery at every day preceding behavioral testing, animals were subject to a food restriction protocol (12-14 g per day) with ad libitum water availability. Ad libitum water and food were available for the rest of the study.

\section{Preoperative behavioral training on the CatWalk}

At the age of 7 weeks, the animals were put into individual housing and a two-week training period was started for the CatWalk gait analysis. The CatWalk analysis and the required training protocol has been described elsewhere [11,14]. Briefly, the CatWalk system consists of a glass runway which contains light from a white fluorescent tube. Internal reflection causes the light to be restricted to the glass surface plate under normal circumstances. However, when an object touches the glass surface, the light exits the glass, thereby only illuminating the contact area. Hence, placement of the rat paws on the glass floor, lights up the corresponding areas on the glass floor. The run of the animal across the glass runway is detected by a video camera (Sony 3CCD Color Video Camera; DXC-990/990P) positioned underneath the glass plate. The signal is digitized (50 half-frames/sec) by an S-RGB frame grabber (Matrix Vision $\mathrm{GmbH}$, Oppenheimer, Germany). The data are acquired, compressed, stored, and eventually analyzed by the CatWalk software program [14]. This way, a wide range of gait parameters can be measured from each run a rat makes across the glass runway. During a two-weeks-period in which a 12-gram per day food deprivation protocol was used for motivating the animals, the animals were trained to make consecutive runs over a glass runway without any hesitations. Each run was rewarded by foot pellets (Noyes Precision pellets PJPPP-0045; Sandown Chemical Ltd., Hempaton, U.K.) which were placed at the end of the runway. The animals finished their training period within two weeks and were then able to make at least three consecutive runs without interruptions. Importantly, the gait velocity was controlled by the fact that training of the animals on the CatWalk resulted in runs with a stable crossing time of the runway between one and two seconds.

\section{Surgery}

At the age of 9 weeks, the 36 animals were divided into two groups: one resection group ( $n=25$; average weight: $272 \pm 2.3 \mathrm{~g}$ ) and one sham group ( $n=11$; average weight $267.9 \pm 3.8 \mathrm{~g}$ ). Approximately 30-60 min before surgery the animals received an intraperitoneal injection with Buprenorfine (Temgesic 0.1 mg/kg body weight; Schering-Plough, Utrecht, The Netherlands). Then, anesthesia was induced with 5\% isoflurane in a 1:1 mixture of air and 100\% oxygen at 
a flow of $250 \mathrm{ml} / \mathrm{min}$ using an Inventor $400^{\circledR}$ injection system vaporizer (Zevenaar, The Netherlands) with an open mask system. Anesthesia was maintained with $2 \%$ isoflurane using the same carrier gases as described above. An ophthalmic ointment (Visagel, Eurovet, Bladel, The Netherlands) was applied to the eyes to prevent drying during surgery. The operation area overlying the left sciatic nerve was freed from hair and the skin scrubbed with Bethadine before making an incision of the skin. Under optical magnification $(\times 25)$ the left sciatic nerve was exposed taking care not to damage the overlying musculature. Only in animals of the resection group, a 1-cm long piece of the left sciatic nerve was resected using a specially adapted forceps to fix the sciatic nerve over a 1-cm distance and cutting the nerve at both ends of this special forceps. The most proximal end of the exerted 1-cm sciatic nerve segment was located immediately distal to the posterior biceps semitendinosus, where the sciatic nerve is still monofascicular [15]. Next, the skin of the animals was sutured with non-absorbable 3/0 polyester threads and the animals were returned to their home cages to recover from surgery. Sham-operated animals were treated identically as animals from the resection group with the exception of leaving the sciatic nerve intact.

\section{Functional testing}

All testing or analysis was carried out blinded to the groups of animals when possible. However, the morphological anomalities developing in only resected animals hampered the investigators' blindness to groups. Preoperatively, basal values of the CatWalk gait analysis were obtained and after surgery behavioral testing was performed at days post-operative (DPO) 3 , 5, 7 and then once weekly until DPO56.

\section{Autotomy score}

Spontaneous pain-like behavior was scored as previously described [16] since it may affect behavioral testing. Briefly, after observing closely the hindpaws of the animals an autotomy score was given according to the following criteria: A score of 1 for removal of one or more nails and an additional score of 1 was added for each half digit attacked. Hence, removal of all digits resulted in a score of 11. Throughout the present study, the maximum autotomy score observed was 7.

\section{CatWalk gait analysis}

The setting of the CatWalk system was as mentioned above (see preoperative behavioral training on the CatWalk). The CatWalk gait analysis appreciates a substantial amount of gait parameters, both dynamic and static. Some of these parameters, including mean intensity of paw placement, stance duration and swing duration of the hindpaw, may not only involve motor control of the animal, but have also been linked to neuropathic pain [17]. However, 
most parameters may be regarded as strictly motor-related. For reliable comparison between the gait parameters of animals in the resection group and those in the sham group it is of utmost importance that there is no difference in speed of gait between the two groups of animals. This is important because speed of gait is known to strongly affect gait parameters $[8,11]$. Table 1 shows an explanatory overview of the evaluated gait parameters.

\section{Data presentation and statistical analysis}

Data on animal weight and, autotomy are presented as mean \pm SEM. Data on the other behavioral parameters are shown as ratio between the ipsilateral (left) hindpaw and the contralateral (right) hindpaw. Statistical analysis was done using analysis of variance (ANOVA)

Table 1. Explanation of CatWalk parameters

\begin{tabular}{|c|c|}
\hline CatWalk parameter & Explanation \\
\hline Base-of-support (BOS) & Distance between two hindpaws, as measured perpendicular to the waking direction. \\
\hline Stride Length & $\begin{array}{l}\text { Distance between the placement of a hindpaw and the subsequent placement of the } \\
\text { same paw. }\end{array}$ \\
\hline Box length & $\begin{array}{l}\text { Length of the box which is artificially placed around the paw prints by the CatWalk } \\
\text { software program. }\end{array}$ \\
\hline Box width & $\begin{array}{l}\text { Width of the box which is artificially placed around the paw prints by the CatWalk so- } \\
\text { ftware program. }\end{array}$ \\
\hline Maximum area & $\begin{array}{l}\text { Total surface area of the glass floor contacted by the hindpaw at the moment of ma- } \\
\text { xium paw-floor contact during the stance phase of the hindpaw. }\end{array}$ \\
\hline Print area & $\begin{array}{l}\text { Total surface area of the glass floor contacted by the hindpaw during the complete } \\
\text { stance duration. }\end{array}$ \\
\hline Mean intensity & Mean intenstiy of the pixels forming the maximum area. \\
\hline Stance duration & Time of contact of the hindpaw with the glass floor. \\
\hline Swing duration & Time that the hindpaw is not in contact with the glass floor. \\
\hline Regularity Index (RI) & $\begin{array}{l}\text { An index for the degree of interlimb coordination during gait, as measured by the num- } \\
\text { ber of normal step sequence patterns (NSSP), multiplied by four (number of paws), divi- } \\
\text { ded by the number of paw placements, and multiplied by 100\%; RI = (NSSP x4)/ num- } \\
\text { ber of paw placements x 100\%. With respect to the RI, six NSSP have been previously } \\
\text { described (Cheng et al ., 1997) and involve cruciate, alternate, and rotary step patterns. }\end{array}$ \\
\hline Phase lags & $\begin{array}{l}\text { Parameters that appreciate the timing relationships between paw placements. The time } \\
\text { of initial contact of one paw (the Target) is related (expressed as a percentage) to the stri- } \\
\text { de length of another paw (the Anchor). Phase lags can be calculated between the paws } \\
\text { of the same girdle (forepaws or hindpaws), between paws on the same side (ipsilateral } \\
\text { left or ipsilateral right), and between diagonal paws (opposite forepaw/hindpaw). }\end{array}$ \\
\hline
\end{tabular}


with repeated measures over time. In case of overall group differences, additional non-paired t-tests were performed to test for group differences per individual time point. A $p$-value below 0.05 was regarded as statistically significant.

\section{Results}

All 36 animals recovered fast from the surgery and were active from DPO1 onwards. Noticeably, animals from the resection group developed joint contracture. Especially at the end of the study, this was evident in all 25 animals of the resection group, where the angle of ankle joint bending without resistance (Figure 1A,B) was much smaller in the affected than in the unaffected paw. In addition, animals with nerve resection, showed a strong decrease in toe spreading (Figure 1C,D) of the affected as compared to the unaffected paw.
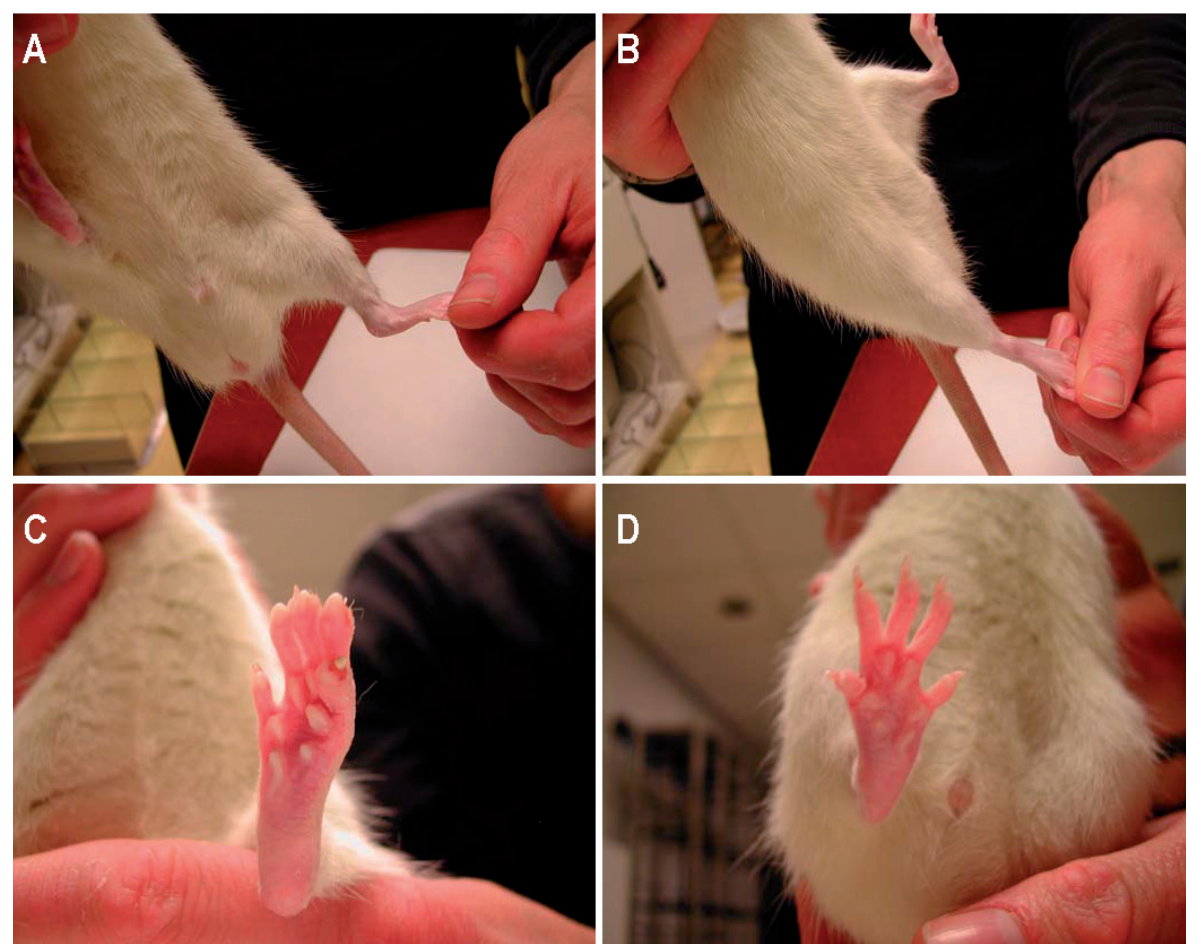

Figure 1. Qualitative observations after sciatic neurotmesis. After resecting a 1-cm long segment of the left sciatic nerve, a contracture (A) and a reduced toe spreading (C) was observed. In this figure, a representative animal is shown that started as one of the 25 animals within the resection group at the beginning of the experiment. In figures $A$ and $C$ the ipsilateral (i.e. left) hindpaw is shown; in figures $B$ and $D$ the contralateral (i.e. the right hindpaw). Note that in figures $A$ and $B$ the bending of the ankle joint until the point of resistance is shown. Also noteworthy is the attacked outer toe of the left paw as seen in figure $C$. 


\section{Autotomy}

All animals in the resection group $(n=25)$ showed autotomy at the end of the study. Figure 2A shows the average autotomy score of these 25 animals, which significantly differs from sham operated animals that never showed signs of autotomy (Table 2). Noticeably, animals with autotomy scores above 1 always attacked their outer toes and never their inner ones. Out of the 25 animals in the resection group, 13 animals displayed an autotomy score above 1 at DPO56. The autotomy prevalence in the resection group is given in Figure 2B.
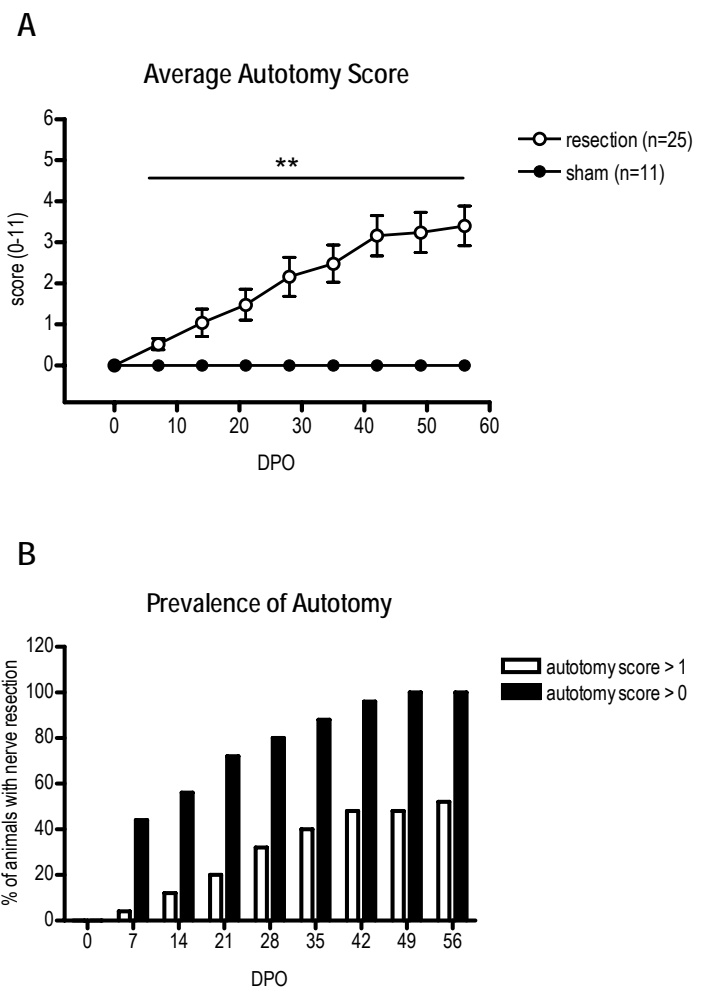

Figure 2. Autotomy after sciatic neurotmesis. Spontaneous pain occurred early after resection and gradually increased over time. Figure A gives an average autotomy score of the complete resection group of 25 animals, whereas figure B shows the percentage of these 25 animals with autotomy scores above 0 or 1 (black and white bars, respectively). Note that only animals with an autotomy score of 0 or 1 ( $n=11$ in both resection and sham groups) were included in the behavioral analyses of this study. DPO, days post-operative; ${ }^{* *}, \mathrm{p}<0.01$ (at individual time points between the resection and sham groups). 


\section{Gait analysis}

Although all 36 animals were trained on the CatWalk, two of these animals (assigned to the nerve resection group via ad random selection) did not learn to cross the CatWalk runway without hesitations and were therefore excluded from any CatWalk data. All 34 animals remaining were able to make CatWalk runs without hesitations from DPO1 onwards. Although all animals used all four hindpaws on the CatWalk, five animals did not display the affected hindpaw on the CatWalk at DPO1, probably due to a reduced body weight on this paw below the detection threshold. Therefore, DPO1 was excluded from the CatWalk analysis leaving a total of 23 animals with nerve resection and 11 with a sham operation in the CatWalk analysis from DPO3 onwards. However, autotomy scores above 1 as observed in 13-out-of-24 animals at DPO56 reflect missing digits, which may obviously affect CatWalk parameters box length, box width, maximum area, and print area. Therefore, only animals with an autotomy score of maximally 1 ( $n=11$ per group) were included in the analysis of these four CatWalk parameters.

With respect to reliable comparison of gait parameters between animals with nerve resection and sham operated animals, two aspects are of high importance: body weight and speed of gait. First, body weight may seriously confound interpretations about CatWalk parameters such as the mean intensity parameter of gait. However, the overall averaged body weight did not differ between the sham group and the resection group (either consisting of all 23 animals or of 11 animals without missing digits; Figure 3A, B). Nevertheless, there were overall differences over time (Table 2). Since also a time $x$ group effect was found for body weight (Table 2), an additional $t$-test was performed per time point. Between the 11 sham animals and the 11 animals with nerve resection only a significant difference was found at DPO56 $(p<0.05)$, whereas the 11 sham animals already differed from the 23 animals in the resection group from DPO42 onwards $(p<0.05)$. Second, the speed of gait may have strong influence on many gait parameters including print area, stance and swing duration. However, also with respect to crossing time of the CatWalk (directly related to speed of gait), no significant differences were found between animal groups (Figure 3C, D). There was, however, a significant difference in overall crossing time over time (Table 2). 
Table 2. Statistics related to body weight, autotomy, and CatWalk parameters

\begin{tabular}{|c|c|c|c|}
\hline \multirow[b]{2}{*}{ Parameter } & \multicolumn{3}{|c|}{ Statistics } \\
\hline & Group & Time & TimexGroup \\
\hline Average Autotomy Score & $F(1,34)=16.5 ; p<0.01$ & $F(8,34)=10.2 ; p<0.01$ & $F(8,34)=10.2 ; p<0.01$ \\
\hline \multicolumn{4}{|l|}{ Body Weight } \\
\hline (23 resected vs 11 controls) & n.s. & $F(11,32)=354.1 ; p<0.01$ & $F(11,32)=5.2 ; p<0.01$ \\
\hline (11 resected vs 11 controls) & n.s. & $F(11,20)=285.3 ; p<0.01$ & $F(11,20)=3.7 ; p<0.01$ \\
\hline \multicolumn{4}{|l|}{ Crossing Time } \\
\hline (23 resected vs 11 controls) & n.s. & $F(10,32)=6.7 ; p<0.01$ & n.s. \\
\hline (11 resected vs 11 controls) & n.s. & $F(10,20)=5.2 ; p<0.01$ & n.s. \\
\hline Base-of-support (BOS) & $F(1,32)=45.2 ; p<0.01$ & $F(10,32)=15.0 ; p<0.01$ & $F(10,32)=7.5 ; p<0.01$ \\
\hline Box Length & $F(1,20)=250.4 ; p<0.01$ & $F(10,20)=6.1 ; p<0.01$ & $F(10,20)=4.2 ; p<0.01$ \\
\hline Box Width & $F(1,20)=643.4 ; p<0.01$ & $F(10,20)=28.2 ; p<0.01$ & $F(1,20)=26.7 ; p<0.01$ \\
\hline Maximum Area & $F(1,20)=14.4 ; p<0.01$ & $F(10,20)=13.2 ; p<0.01$ & $F(1,20)=579.3 ; p<0.01$ \\
\hline Print Area & $F(1,20)=819.7 ; p<0.01$ & $F(10,20)=14.4 ; p<0.01$ & $F(1,20)=15.1 ; p<0.01$ \\
\hline Regularity Index (RI) & n.s. & n.s. & n.s. \\
\hline \multicolumn{4}{|l|}{ NSSP } \\
\hline Alternate Aa & $F(1,32)=65.4 ; p<0.01$ & $F(10,32)=8.1 ; p<0.01$ & $F(10,32)=12.8 ; p<0.01$ \\
\hline Alternate Ab & $F(1,32)=28.1 ; p<0.01$ & $F(10,32)=5.8 ; p<0.01$ & $F(10,32)=3.3 ; p<0.01$ \\
\hline Cruciate Ca & $F(1,32)=89.2 ; p<0.01$ & $F(10,32)=5.8 ; p<0.01$ & $F(10,32)=2.5 ; p<0.01$ \\
\hline Cruciate Cb & $F(1,32)=11.4 ; p<0.01$ & n.s. & $F(10,32)=2.4 ; p<0.05$ \\
\hline Rotary Ra & n.s. & n.s. & n.s. \\
\hline Rotary Rb & n.s. & n.s. & n.s. \\
\hline \multicolumn{4}{|l|}{ Phase Lags } \\
\hline Diagonal LF-RH & n.s. & n.s. & n.s. \\
\hline Diagonal RF-LH & n.s. & n.s. & n.s. \\
\hline Girdle LF-RF & n.s. & n.s. & n.s. \\
\hline Girdle LH-RH & $F(1,32)=386.3 ; p<0.01$ & $F(10,32)=15.8 ; p<0.01$ & $F(10,32)=18.0 ; p<0.01$ \\
\hline Ipsilateral LF-LH & $F(1,32)=194.4 ; p<0.01$ & $F(10,32)=3.6 ; p<0.01$ & $F(10,32)=3.6 ; p<0.01$ \\
\hline Ipsilateral RF-RH & n.s. & $F(10,32)=4.8 ; p<0.01$ & n.s. \\
\hline Mean Intensity & $F(1,32)=182.9 ; p<0.01$ & $F(10,32=5.0 ; p<0.01$ & $F(10,32)=6.1 ; p<0.01$ \\
\hline Stance Duration & $F(1,32)=806.5 ; p<0.01$ & $F(10,32)=22.8 ; p<0.01$ & $F(10,32)=21.8 ;<0.01$ \\
\hline Swing Duration & $F(1,32)=144.0 ; p<0.01$ & $F(10,32)=5.7 ; p<0.01$ & $F(10,32)=6.5 ; p<0.01$ \\
\hline
\end{tabular}


A

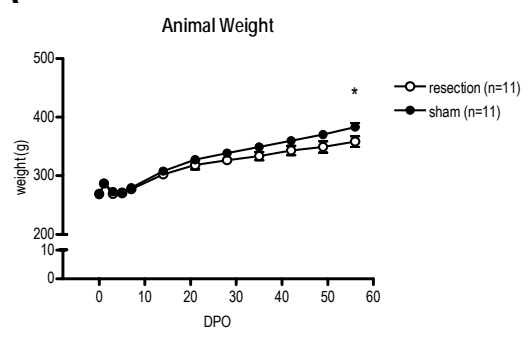

C

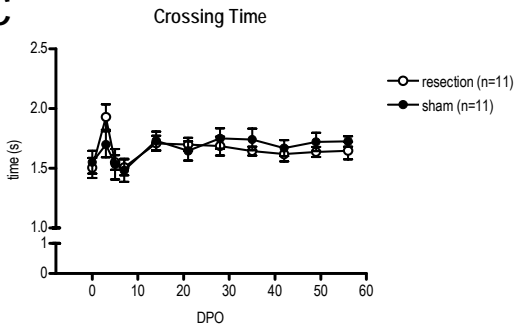

B

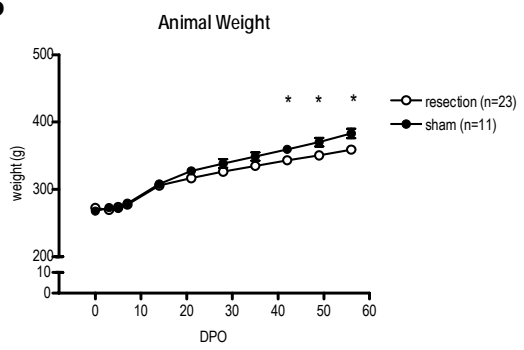

D

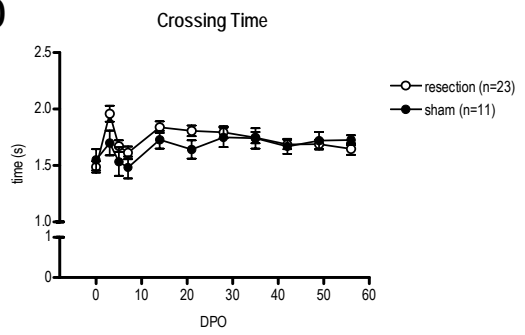

Figure 3. Main confounding factors for CatWalk parameters: body weight and crossing time. Body weight and the time needed for the animal to cross the CatWalk runway can influence several CatWalk parameters. Based on this, group comparison is allowed, because there are no group differences for body weight (A) and crossing time (B). However, at the last time point, there is a significant difference between both animal groups (indicated with an asterisk). Thus, the behavioral data on this latest time point may be influenced by body weight. DPO, days post-operative; ${ }^{*}, \mathrm{p}<0.05$.

\section{General parameters: Base-of-support (BOS) and stride length}

General gait parameters that may be altered as a result of compromised locomotion after nervous system injury include the base of support (BOS) [18] and stride length of the hindpaws [12]. In addition, nerve injury may result in an impaired placement of the paw on the glass plate, due to an altered use of the plantar surface of the paw. These phenomena, then, result in alterations in parameters such as box length, box width, maximum area, and print area. Resection-induced alterations in all-but-one of these parameters are shown in Figure 4 (A, B). Whereas stride length is not impaired after nerve resection, all others are. Overall there are strong changes with respect to time, group, and timexgroup (Table 2). And also here, animals in the resection group differ from those in the sham group from DPO1 onwards until the last time point measured (DPO56), with respect to all five parameters $(p<0.01)$. In addition, nerve injury may result in an impaired placement of the paw on the glass plate, due to an altered use of the plantar surface of the paw. These phenomena, then, result in alterations in parameters such as box length, box width, maximum area, and print area. Resection-induced alterations in all of these parameters are shown in Figure $4(\mathrm{C}-\mathrm{F})$. Overall there are strong 
changes with respect to time, group, and time x group (Table 2). And also here, animals in the resection group differ from those in the sham group from DPO1 onwards until the last time point measured (DPO56), with respect to all parameters $(p<0.01)$.

\section{Coordination-related gait parameters}

The most-frequently used coordination parameter in the CatWalk is the regularity index (RI), which indicates the regularity or degree in which the animal uses NSSP. Hence, RI does not take timing of paw placements into account, but merely the order of paw placements. Figure 5 shows that the RI, overall, is not affected by time or by group. Nevertheless, animals

A

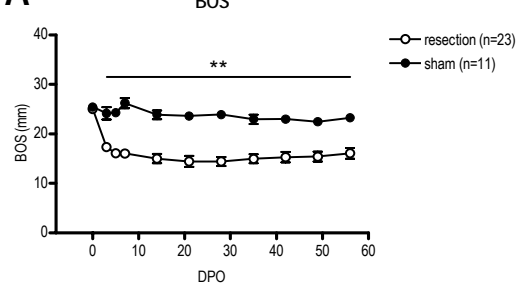

C

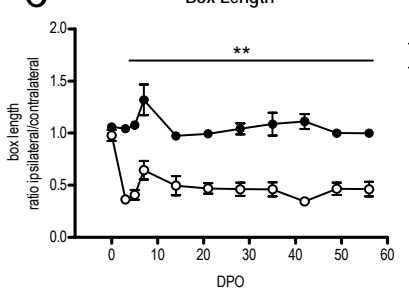

E

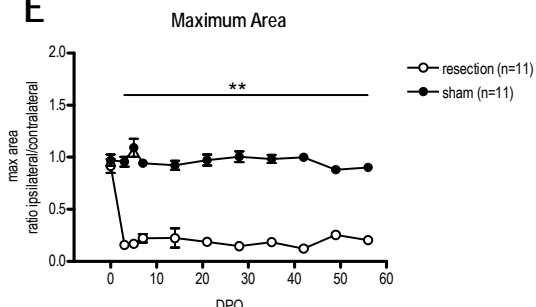

B Stride Length

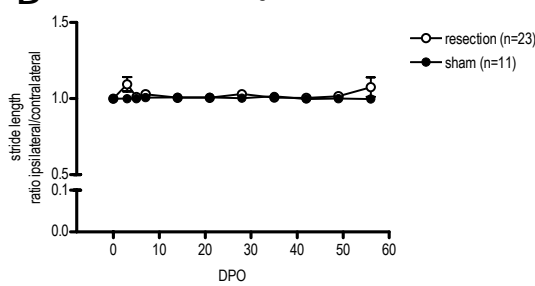

D Boxwidth

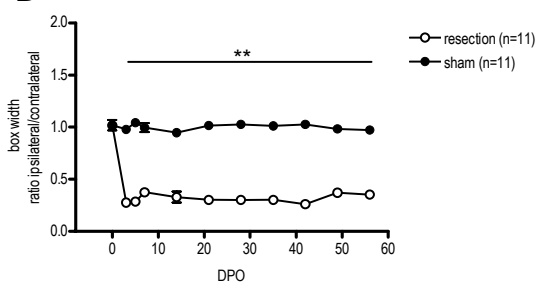

F

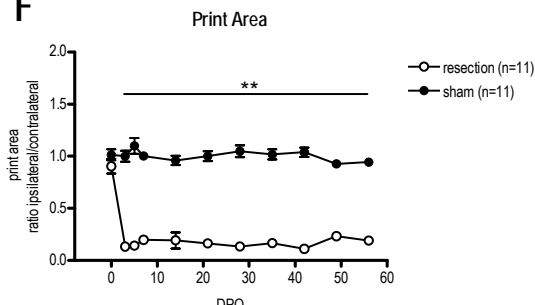

Figure 4. General gait parameters. Although stride length of the hindpaws (B) is not affected after sciatic nerve resection, BOS is significantly reduced from DPO1 to a level of approximately 50\%, which is maintained until the end of the study (A) Also the contact of the paw with the floor is reduced, reflected with decreased box length, box width, maximum area, and print area (C-F) starting immediately after resection injury. BOS, base-of-support; DPO, days post-operative; **, $p<0.01$ (at individual time points between the resection and sham groups). 
may maintain a normal RI, while switching in the use of the various NSSP. Figure 6 gives an overview of the six NSSP according to Cheng and colleagues [19]. After resection, animals almost completely stopped using the alternate Aa (Figure 6A; Table 2) and cruciate Ca patterns (Figure 6C; Table 2). T-testing at individual time points showed that the resection group differed from the sham group at all post-operative time points. In contrast to alternate $\mathrm{Aa}$ and cruciate Ca patterns, the use of alternate $\mathrm{Ab}$ and cruciate $\mathrm{Cb}$ patterns increased (Figure $6 \mathrm{~B}$ and $\mathrm{D}$, respectively). Overall, significant differences in alternate $\mathrm{Ab}$ and cruciate $\mathrm{Cb}$ pattern were detected over time, group, and time x group (Table 2). Statistically significant group differences at individual time points were detected at all post-operative time points for the alternate Ab pattern and at DPO5-14, and 42 for the cruciate Cb pattern. The use of the rotary $\mathrm{Ra}$ and $\mathrm{Rb}$ patterns were already minimal and remained so in both resection and sham groups (Figure 6E, F).

Other coordination parameters are represented by the phase lags, which in contrast to the RI and the six NSSP determine the paw placements with respect to timing (Figure 7). ANOVA testing did not show any overall differences in the phase lags of the diagonals (Figure 7A,B) between the resection and the sham groups. The phase lags of the girdle were affected the most (Figure 7D), where the timings between positioning of the hindpaws differed strongly over time, group, and groups over time (Table 2). No overall differences were detected in the phase lags between the forepaws (Figure 7C). With respect to the ipsilateral phase lags, timing between the paws on the left side of the animals (Figure 7E) showed overall significant differences over time, group, and time $x$ group (Table 2). The animals in the resection group and those in the sham group significantly differed at this parameter for all post-operative time points. The phase lags of the paws on the right side of the animal only significantly differed over time (Figure 7F; Table 2).

$\mathbf{R}$

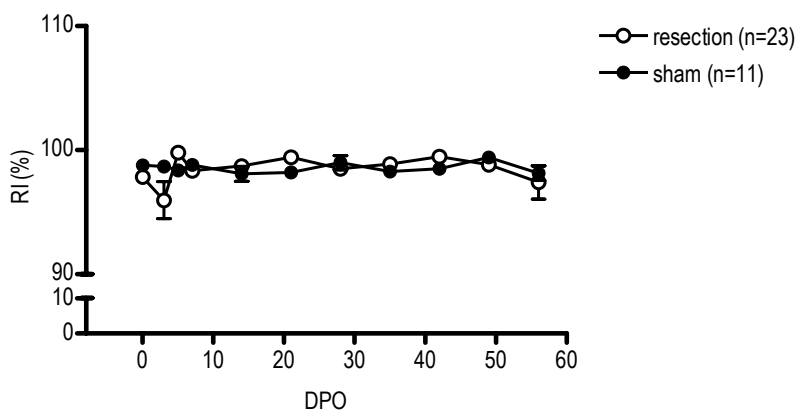

Figure 5. Degree of normal step sequence patterns used: regularity index. The use of normal step sequence patterns, according to Cheng et al., 1997 is unchanged after sciatic nerve resection as shown by an unchanged RI. RI, regularity index; DPO, days post-operative. 
A

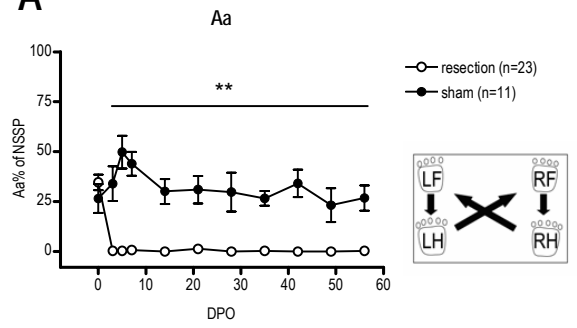

C

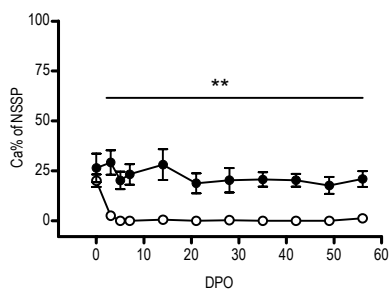

E

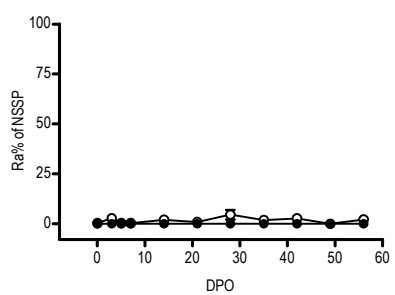

B

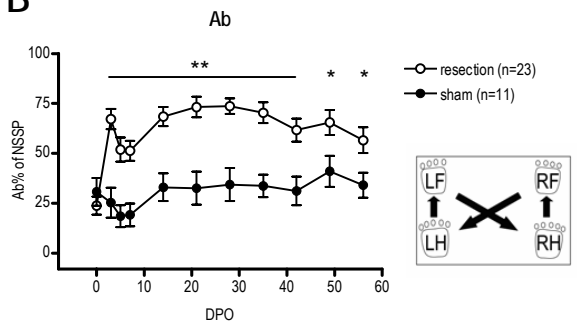

D
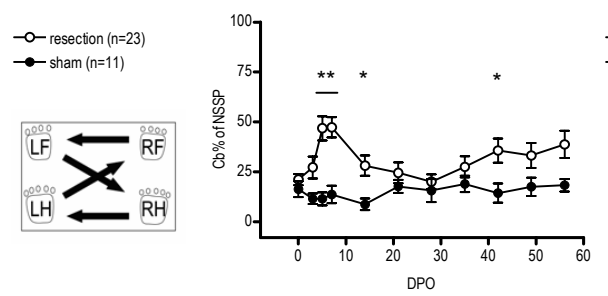

-0 - resection ( $\mathrm{n}=23)$

$\rightarrow \operatorname{sham}(\mathrm{n}=11)$

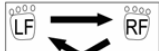

$\mathrm{LH} \longrightarrow \mathrm{RH}$

Figure 6. Use of normal step sequence patterns for coordination. Although the degree of NSSP used is unchanged after neurotmesis, the use of individual NSSP is changed. Figures A-D show that the use of the Aa and Ca patterns is almost completely abolished, whereas the $\mathrm{Ab}$ and $\mathrm{Cb}$ patterns are increasingly used. Ra and Rb patterns are used to only a minor extent, both before and after surgery. The small insets indicate the order of paw placements. NSSP, normal step sequence patterns; DPO, days post-operative; *, $\mathrm{p}<0.05 ;{ }^{* *}, \mathrm{p}<0.01$ (at individual time points between the resection and sham groups). 
A
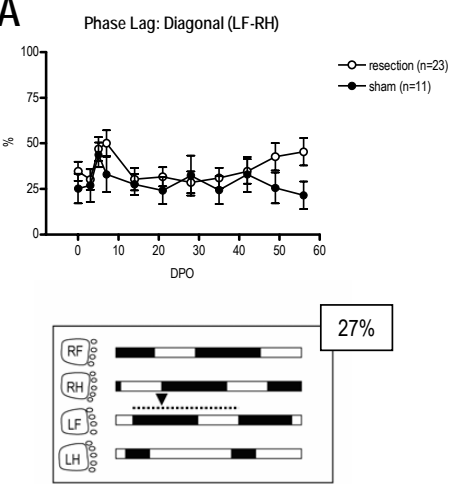

C
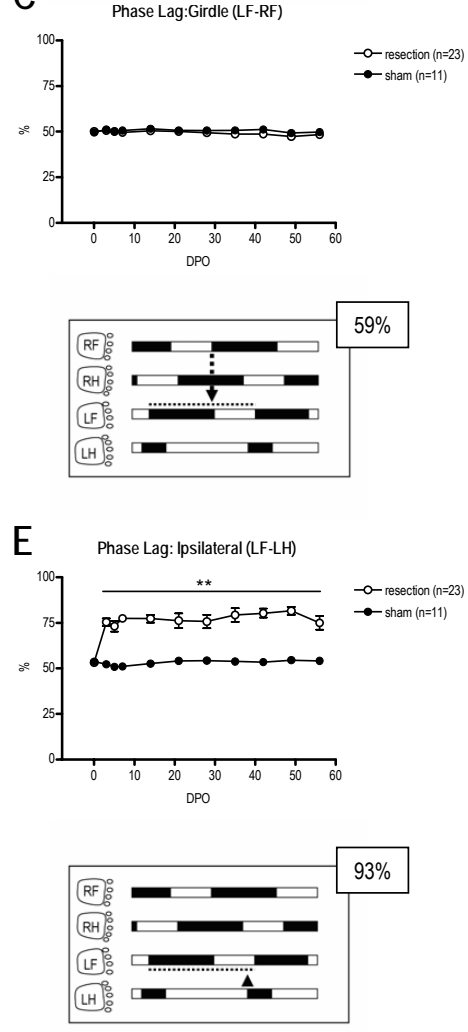

B
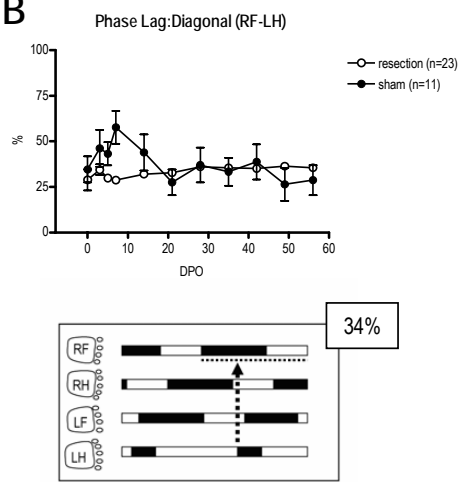

D
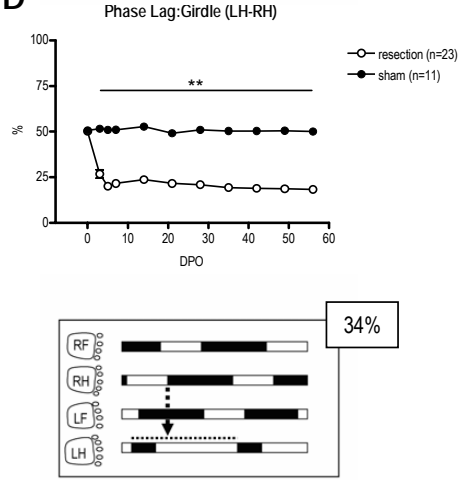

F

F PhageLag. lpsildted (RFFH)
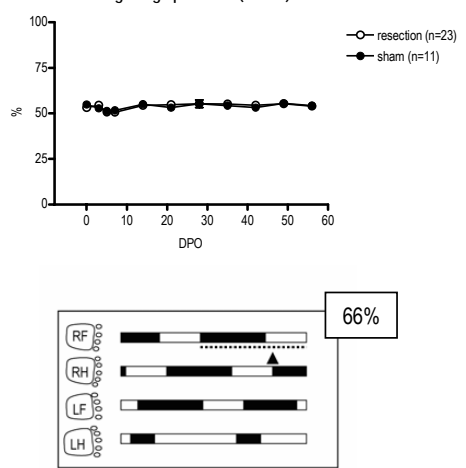

Figure 7. Phase lags as a measure to evaluate coordination with respect to timing of paw placements. The most obvious changes in timing of paw placements are detectable in the phase lags of the girdles and in the ipsilateral phase lag between the left forepaw and the left hindpaw. As a result of neurotmesis of the left sciatic nerve, the left hindpaw is placed later than normal, which accounts for most of these effects. The insets below the figures show a representative illustration of the phase lags in animals of the resection group (the thin dotted line represents the step cycle duration of the associated paw, whereas the thick dotted line indicates the \% of the step cycle (highlighted) at which another paw is placed). LF, left forepaw; LH, left hindpaw; RF, right forepaw; RH, right hindpaw; DPO, days post-operative;,$p<0.05 ;{ }^{* *}, p<0.01$ (at individual time points between the resection and sham groups). 


\section{Pain-related gait parameters}

The CatWalk gait parameters mean intensity, stance duration, and swing duration have previously been linked to mechanical hyperalgesia [17] and were strongly affected in this study (Figure 8A-C). All three parameters significantly differed overall for time, group, and time $\times$ group (Table 2). For all postoperative time points animals with nerve resection differed significantly from the sham operated animals with respect to all three gait parameters, as observed after t-testing $(p<0.01)$.
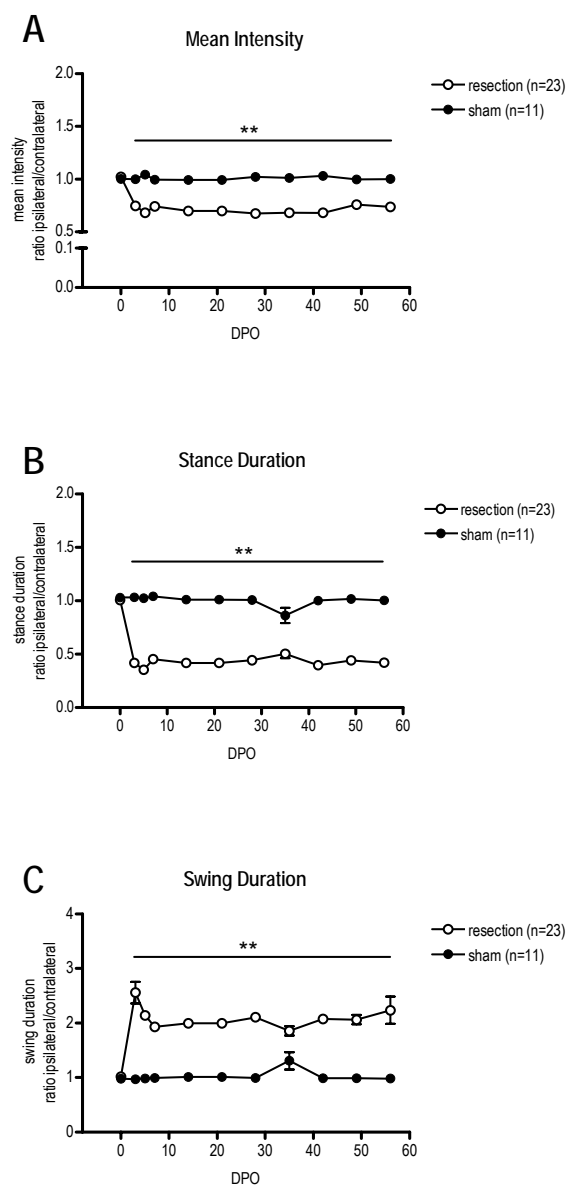

Figure 8. Pain-related CatWalk parameters. Mean intensity, stance duration and swing duration have all been correlated with the development of mechanical hyperalgesia after chronic constriction injury of the sciatic nerve (Vrinten et al., 2003). Figures A-C show early onset impairments in these parameters. DPO, days post-operative; ${ }^{* *}, p<0.01$ (at individual time points between the resection and sham groups). 


\section{Discussion}

We here showed that the CatWalk gait analysis is an automated quantitative method which can be used to objectively detect a large set of both dynamic and static gait changes associated with adult rat sciatic nerve resection. Although mirror-image pain behavior may arise from the body regions contralateral to the injured side [20], the lack of such behavior allowed us to use the contralateral paw as a control in this study.

The most well-known motor changes after neurotmesis are described in the SFI and include an altered print length of the affected hindpaw and decreased toe spreading [5]. The SFI has been proven very valuable not only in detection of gait deficits after neurotmesis, but also in demonstrating recovery of these specific gait deficits as a result of axon regeneration after sciatic crush injury [5]. Nevertheless, many more gait alterations have been reported after neurotmesis which are not taken into consideration in the SFI, including gait-stance duration [8]. Gait deficits after neurotmesis are, thus, do not only concern static, but also dynamic gait parameters. Hence, the CatWalk gait analysis, which was proven useful to detect static and dynamic gait changes after experimental spinal cord injury, was used to identify a wide range of gait deficits after neurotmesis. Importantly, gait velocity, which strongly affects the variations in gait parameters, is easily controlled using the CatWalk [14] by training the animals to make runs with a stable crossing time. The most apparent changes after neurotmesis detected using the CatWalk are [1] a smaller base-of-support (BOS), [2] a smaller contact area of the affected paw with the floor, and [3] modified coordination. First, the decreased BOS was rather surprising because other types of neural injury, i.e. spinal cord injury, resulted in an increased BOS $[14,21,22]$, which has been explained as adaptations for an instable gait [13]. The decreased BOS after sciatic nerve neurotmesis may, therefore, rather represent a separate motor dysfunction than an adaptive response of the animal to other dysfunctions. Second, the animals did not use the complete plantar surface of the paw for walking after neurotmesis. Instead, the animals started using mainly the heel of the paw and the front part of the paw including the toes was often absent in the print (irrespective of autotomy). The preferential use of the heel of the paw is reflected in parameters, such as box length, box width, maximal area, and print area, which were robustly decreased to values around $15-50 \%$ in the affected paw as compared to the contralateral paw. Although the previously reported increased print length of the affected hindpaw as measured by the SFI contrasts the decreased print parameters measured by the CatWalk, both measures are likely to represent the same phenomenon [5]. The overlapping feature is the additional use of the heel during walking. Nevertheless, the CatWalk clearly differentiates in the way of use of the paw in which parts of the paw that barely carry body weight are below the detection threshold (front part of the paw), whereas the SFI via walking track analysis does not differentially assess parts of the paw with differences in body weight support. The impairments in these paw contact size related gait parameters likely represent motor dysfunctions instead of compensatory behavior. Third, alterations in coordination-related parameters were observed in animals with sciatic nerve resection. The 
regularity of paw placements, i.e. the order in which the paws are placed, was unchanged. This is understandable since coordination is primarily controlled by the central pattern generators in the spinal cord [23], which are undamaged after sciatic nerve resection. Nevertheless, the animals started to highly prefer two out of the six available normal step sequence patterns [19], and the timing of the paw placements was changed. After neurotmesis, the affected left hindpaw was always preceded by the most distant paw, i.e. the right forepaw. This may suggest that in order to maintain a balanced gait, the animal shows, at the time of using the affected hindpaw, preference for having the center of gravity as far away as possible from this affected paw. Furthermore, the affected left hindpaw was positioned later than normally and this resulted in a smaller phase lag of the girdle paw pair (left hindpaw - right hindpaw) and in a higher phase lag of the ipsilateral paw pair (left forepaw - left hindpaw).

Since neuropathic pain is also known to develop after neurotmesis, differences in gait parameters may not only reflect motor-related impairments, but also pain-induced gait compromises. Especially three CatWalk parameters, i.e. mean intensity, stance duration, and swing duration, have been found to correlate with mechanical hyperalgesia after chronic constriction of the sciatic nerve [17]. At least a part of the alterations in these CatWalk parameters are related to a decreased weight load on the affected paw, which has previously been linked to pain $[24,25]$. Nevertheless, there are indications that the gait alterations found after sciatic nerve neurotmesis were motor-related rather than pain-related. Mechanical hyperalgesia was found to develop after a delay of two weeks after neurotmesis (data not shown). This contrasts the early onset of gait impairments after neurotmesis. From this we can conclude that mean intensity, stance duration, and swing duration are not necessarily directly related to tactile hyperalgesia based on von Frey testing. In our view, functional impairments such as mean intensity of the hindpaws (possibly due to a decreased weight bearing) in pain models without an injured motor system [26] may be purely related to pain behavior. However, as soon as motor axons are damaged, the behavioral effects may be a compromise between pain-related and motor-related alterations. Although also chronic nerve constriction injury is known to partially damage the motor system [27], the complete loss of motor axons in the sciatic nerve after neurotmesis may partly explain the lack of coexisting impairments in CatWalk parameters and mechanical hyperalgesia (not shown).

At present, the SFI is still the most widely used index to assess behavioral impairment after neurotmesis and functional benefit of interventions to repair neurotmesis. The strength of the SFl is mostly thought to lie in the use of a single index indicating sciatic nerve functionality using three to four meaningful gait parameters. Calculation of the SFI includes print length and toe spreading measurements combined with weighing factors to end up with a value of $-100 \%$ after complete transection of the sciatic nerve [5]. There are, however, a couple of drawbacks with respect to the SFI. First, the SFI is limited by a variety of neurotmesis-induced phenomena including unclear paw prints as a result of contractures, autotomy, and tail dragging [7] and the SFI may be confounded by variations in gait velocity. Second, the SFI is restricted 
to measuring static gait parameters, whereas neurotmesis is also known to result in altered dynamic gait parameters $[8,9]$. Third, any small improvement in print length or toe spreading may be missed using the SFI since these measures are only part of a bigger formula.

Although the print size measures of the CatWalk are, obviously, also impeded by autotomy, the CatWalk allows for reliable gait velocity-controlled assessment of a wide range of both dynamic and static gait changes after neurotmesis, even in the presence of autotomy. The majority of these parameters is strongly impeded after neurotmesis, reaching levels up to $15 \%$ of those in the unaffected paw and it can be ruled out that these changes may be related to changes in gait velocity. Additionally, the impairments are very consistent without signs of recovery, which is obviously related to the fact that a resection gap of $1 \mathrm{~cm}$ prevents recovery. However, the consistency in impairment also indicates that still intact nerves, such as the femoral nerve, does not compensate for the behavioral deficits after sciatic nerve resection. Hence, the main benefit of the CatWalk gait analysis is that it is a single behavioral test which can with a high sensitivity detect a large set of both dynamic and static velocity-controlled gait changes after neurotmesis. It needs to be emphasized, however, that the CatWalk cannot yet be regarded as an alternative for the SFI. The SFI has been shown useful not only in detecting deficits, but also in recovery of gait parameters after neurotmesis. Before adopting the CatWalk system in research on repair after injury, future experiments have to show which parameters are of particular use to detect functional recovery after selective target reinnervation as occurs after crush injury. It may even be possible to calculate a single index for sciatic nerve functioning out of all these latter gait parameters, but it is our belief that the use of each parameter individually improves the sensitivity to detect functional improvements after neurotmesis repair. Comparisons to methods as the SFI or it modifications may prove that the CatWalk can be used as a complementary test, especially in the first weeks after neurotmesis when paw prints are very difficult to distinguish.

In conclusion, although a combination of behavioral tests needs to be used in order to assess recovery of all sciatic nerve functions, the CatWalk gait analysis is a novel test that only by itself allows for the reliable detection of many velocity-controlled dynamic and static gait alterations after neurotmesis. The next step is now to identify which of these many parameters are of particular use to additionally detect functional recovery.

\section{Acknowledgements}

This work was financially supported by DSM Research, Performance Materials (grant ACER 2006-154 to EAJJ and RD). 


\section{References}

1. Noble, J., et al., Analysis of upper and lower extremity peripheral nerve injuries in a population of patients with multiple injuries. JTrauma, 1998. 45(1): p. 116-22.

2. Kelsey, J., et al., Upper extremity disorders: frequency, impact, and cost. London: Chruchill-Livingstone, 1997: p. pp. 26,42.

3. Sunderland, I.R., et al., Effect of tension on nerve regeneration in rat sciatic nerve transection model. Ann Plast Surg, 2004. 53(4): p. 382-7.

4. Belkas, J.S., M.S. Shoichet, and R. Midha, Peripheral nerve regeneration through guidance tubes. Neurol Res, 2004. 26(2): p. 151-60.

5. de Medinaceli, L., W.J. Freed, and R.J. Wyatt, An index of the functional condition of rat sciatic nerve based on measurements made from walking tracks. Exp Neurol, 1982. 77(3): p. 634-43.

6. Bain, J.R., S.E. Mackinnon, and D.A. Hunter, Functional evaluation of complete sciatic, peroneal, and posterior tibial nerve lesions in the rat. Plast Reconstr Surg, 1989. 83(1): p. 129-38.

7. Varejao, A.S., et al., Methods for the experimental functional assessment of rat sciatic nerve regeneration. Neurol Res, 2004. 26(2): p. 186-94.

8. Walker, J.L., et al., Gait-stance duration as a measure of injury and recovery in the rat sciatic nerve model. J Neurosci Methods, 1994. 52(1): p. 47-52.

9. Lin, F.M., et al., Ankle stance angle: a functional index for the evaluation of sciatic nerve recovery after complete transection. J Reconstr Microsurg, 1996. 12(3): p. 173-7.

10. Varejao, A.S., et al., Toe out angle: a functional index for the evaluation of sciatic nerve recovery in the rat model. Exp Neurol, 2003. 183(2): p. 695-9.

11. Koopmans, G.C., et al., The assessment of locomotor function in spinal cord injured rats: the importance of objective analysis of coordination. J Neurotrauma, 2005. 22(2): p. 214-25.

12. Deumens, R., et al., Olfactory ensheathing cells, olfactory nerve fibroblasts and biomatrices to promote long-distance axon regrowth and functional recovery in the dorsally hemisected adult rat spinal cord. Exp Neurol, 2006. 200(1): p. 89-103.

13. Hamers, F.P., G.C. Koopmans, and E.A. Joosten, CatWalk-assisted gait analysis in the assessment of spinal cord injury. J Neurotrauma, 2006. 23(3-4): p. 537-48.

14. Hamers, F.P., et al., Automated quantitative gait analysis during overground locomotion in the rat: its application to spinal cord contusion and transection injuries. J Neurotrauma, 2001. 18(2): p. 187-201.

15. Schmalbruch, H., Fiber composition of the rat sciatic nerve. Anat Rec, 1986. 215(1): p. 71-81.

16. Wall, P.D., et al., Autotomy following peripheral nerve lesions: experimental anaesthesia dolorosa. Pain, 1979. 7(2): p. 103-11.

17. Vrinten, D.H. and F.F. Hamers, 'CatWalk' automated quantitative gait analysis as a novel method to assess mechanical allodynia in the rat; a comparison with von Frey testing. Pain, 2003. 102(1-2): p. 203-9.

18. Koopmans, G.C., et al., Circulating insulin-like growth factor I and functional recovery from spinal cord injury under enriched housing conditions. Eur J Neurosci, 2006. 23(4): p. 1035-46.

19. Cheng, H., et al., Gait analysis of adult paraplegic rats after spinal cord repair. Exp Neurol, 1997. 148(2): p. 544-57. 
20. Milligan, E.D., et al., Spinal glia and proinflammatory cytokines mediate mirror-image neuropathic pain in rats. J Neurosci, 2003. 23(3): p. 1026-40.

21. Joosten, E.A., W.B. Veldhuis, and F.P. Hamers, Collagen containing neonatal astrocytes stimulates regrowth of injured fibers and promotes modest locomotor recovery after spinal cord injury. J Neurosci Res, 2004. 77(1): p. 127-42.

22. Hendriks, W.T., et al., Profound differences in spontaneous long-term functional recovery after defined spinal tract lesions in the rat. J Neurotrauma, 2006. 23(1): p. 18-35.

23. Alford, S., E. Schwartz, and G. Viana di Prisco, The pharmacology of vertebrate spinal central pattern generators. Neuroscientist, 2003. 9(3): p. 217-28.

24. Schott, E., et al., Weight bearing as an objective measure of arthritic pain in the rat. J Pharmacol Toxicol Methods, 1994. 31(2): p. 79-83.

25. Medhurst, S.J., et al., A rat model of bone cancer pain. Pain, 2002. 96(1-2): p. 129-40.

26. Coulthard, P., S.U. Simjee, and B.J. Pleuvry, Gait analysis as a correlate of pain induced by carrageenan intraplantar injection. J Neurosci Methods, 2003. 128(1-2): p. 95-102.

27. Daemen, M.A., et al., Motor denervation induces altered muscle fibre type densities and atrophy in a rat model of neuropathic pain. Neurosci Lett, 1998. 247(2-3): p. 204-8. 



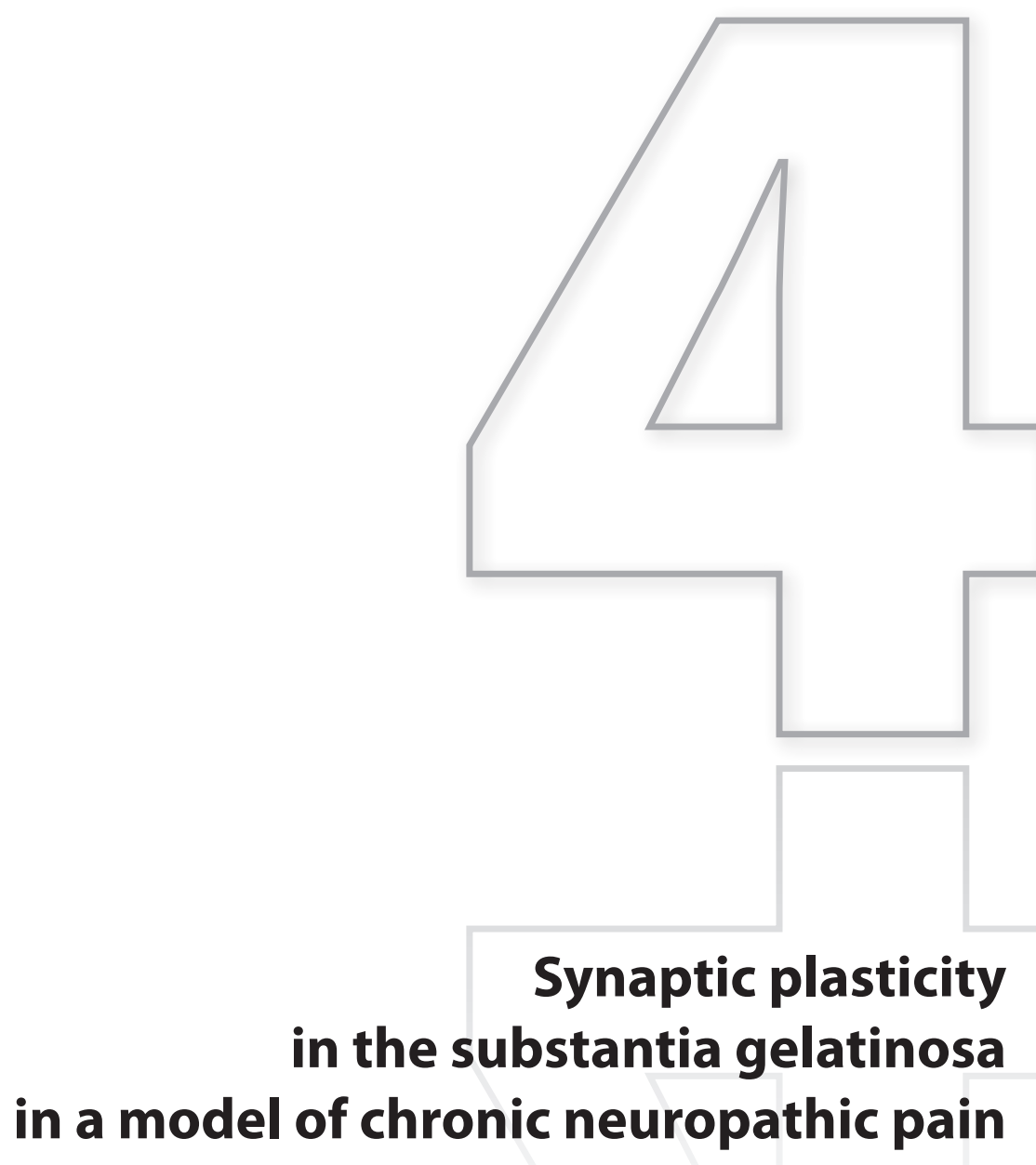

R.J.P. Jaken, E.A.J. Joosten, M. Knüwer, R. Miller,

I. Van der Meulen, M.A.E. Marcus, R. Deumens

Neuroscience Letters (2010) 


\section{Abstract}

Chronic neuropathic pain (CNP) is common after peripheral nerve injuries (PNI), but is rather refractory to available antipain medication. Advances in neuropathic pain research have identified cellular and molecular cues triggering the onset of neuropathic pain, but the mechanisms responsible for maintenance of chronic pain states are largely unknown. Structural changes such as sprouting of injured A-fibres into the substantia gelatinosa of the dorsal horn in the spinal cord have been proposed to relate to neuropathic pain in partial PNI models. Structural changes in central pain networks may also underlie the more persistent CNP following complete sectioning of a peripheral nerve, because this type of injury results in continuous and spontaneous afferent input to the spinal cord, which can trigger central sensitization. In the present study, the left sciatic nerve was completely sectioned and a 1-cm segment was removed to maintain a chronic pathology, whereas the right sciatic nerve was left intact. Mechanical allodynia was measured up to 84 days after injury, after which synaptic changes were studied in the lumbar substantia gelatinosa. The numbers of larger-sized synaptophysinimmunoreactive presynaptic boutons were found to be increased in the substantia gelatinosa ipsilateral to the nerve injury. From these data we conclude that structural synaptic changes within the substantia gelatinosa are present months after complete nerve injury and that this plasticity may be involved in maintaining neuropathic pain states. 


\section{Introduction}

Neuropathic pain (NP) is a major consequence of complete and partial peripheral nerve injuries (PNI) and has a high socio-economic impact. Current pain medication has been proven relatively ineffective in treatment of $\mathrm{NP}$, which may be related to an incomplete knowledge on mechanisms underlying NP. The chronicity of NP may be explained structural changes such as sprouting of non-nociceptive fibers, which was reported to occur within the substantia gelatinosa (SG) following PNI $[1,2,3]$. Moreover, enhanced expression of synaptophysin, a presynaptic vesicle protein, was found within the superficial layers of the lumbar dorsal horn at two weeks after chronic constriction injury of the sciatic nerve, which was paralleled by thermal hyperalgesia [4]. Up to date it is unknown whether persistent synaptic changes occur at months after PNI, which may be related to chronic NP. Therefore, the present study focused on synaptic changes within a key area of the central pain network (i.e. the SG) in a model of persistent neuropathic pain (i.e. complete sciatic nerve injury) [5]. We hypothesized that chronic mechanical allodynia after complete unilateral sciatic nerve injury is related to synaptic changes within the SG.

\section{Materials and methods}

All experimental procedures were performed according to the recommendations of the European Commission (European Communities Council Directive of 24 November 1986; 86/609/ EEC) and protocols were approved by the Committee on Animal Research of the Maastricht University (DEC 2006-054). Eight male Wistar rats (Charles River, The Netherlands) at the age of 9 weeks were used. In this study, every attempt was made to minimize the number of animals and their pain and discomfort. The left sciatic nerve was lesioned as described earlier [6]. In brief, anaesthesia was induced with 5\% isoflurane in a 1:1 mixture of air and 100\% oxygen at a flow of $250 \mathrm{ml} / \mathrm{min}$ using an Inventor 400 vaporizer (Zevenaar, The Netherlands) with an open mask system. Anaesthesia was maintained with $2 \%$ isoflurane using the same carrier gases as described above. Under a $25 \times$ optical magnification, the left sciatic nerve was exposed taking care not to damage the overlying musculature. A 1-cm long piece of the left sciatic nerve was resected using an adapted forceps to fix the sciatic nerve over a 1-cm distance followed by cutting the nerve at both ends, resulting in a sciatic nerve resection injury without spontaneous regeneration. The most proximal end of the exerted 1-cm sciatic nerve segment was located immediately distal to the posterior biceps semitendinosus, where the sciatic nerve is still monofascicular [7]. Next, muscles were sutured with resorbable $4 / 0$ threads and the skin was sutured using nonabsorbable 3/0 polyester threads. The animals were returned to their home cages to recover from surgery.

Tactile nociceptive paw withdrawal thresholds (PWT) were measured using von Frey filaments of varying thickness as described earlier [8]. In brief, before testing the rats were 
allowed to adapt to the experimental conditions. A series of 14 touch-test filaments with increasing stiffness (range of $0.16 \mathrm{~g}$ to $100 \mathrm{~g}$ ) were applied to the medial-to-midplantar area of the hind paws with a pressure causing the filament to bend. The PWT was defined as the force (in grams) of the filament that induced at least three positive paw withdrawal responses out of five filament applications (brisk withdrawal with active attention to the stimulus e.g. licking of the paw, attacking the filament, or clear postural changes). Filaments of increasing force were subsequently tested until the PWT was reached. For this study, we included the values measured before injury (baseline) and at 84 days post operative (DPO), which is the survival time of the animals.

At DPO 84 the rats received an overdose of Nembutal $(150 \mathrm{mg} / \mathrm{kg}$ body weight; i.p. injection). They were transcardially perfused with ice-cold $4 \%$ paraformaldehyde in $0.1 \mathrm{M}$ phosphate buffer ( $\mathrm{pH}$ 7.4). After perfusion, the spinal cords were removed and post-fixed overnight in cold $4 \%$ buffered paraformaldehyde. The following day, the tissue was transferred to $10 \%$ sucrose in PBS $(0.1 \mathrm{M} ; \mathrm{pH} 7.6)$ and kept overnight at $4^{\circ} \mathrm{C}$. Then, the tissue was transferred to $25 \%$ sucrose in PBS and kept for 3 days at $4^{\circ} \mathrm{C}$. After this cryo-protection procedure, $2.5 \mathrm{~cm}$ long spinal cord pieces including the segment innervated by the lesioned nerve (L3-L5) were frozen and stored at $-80^{\circ} \mathrm{C}$. Using a cryostat, the spinal cord pieces were serially cut (transverse sections of $25 \mu \mathrm{m}$ ) and sections of the lumbar spinal cord segments L3, L4 and $L 5$ were obtained. In total, 10 series with an average of 8 sections on each glass side were obtained. The sections were immediately mounted on gelatin chrome-alumn-coated glass slides and stored at $-20^{\circ} \mathrm{C}$. For immunohistochemistry, one glass slide of each animal (one serie) was thawed for at least 2 hours and then washed three times in TBS. Next, slides were incubated for 20 minutes in blocking serum $\left(0.3 \% \mathrm{H}_{2} \mathrm{O}_{2^{\prime}} 0.3 \%\right.$ normal donkey serum in TBS). Slides were incubated overnight with anti-synaptophysin SY38 antibody (1:2,000, Chemicon international) in blocking serum. The following day, slides were washed $3 \times 10$ minutes in TBS. Then, the slides were incubated with biotin-conjugated donkey anti-mouse IgG secondary antibody (1:400, Sanbio) in TBS for 1 hour at room temperature. Following another wash of $3 \times 10$ minutes in TBS, slides were incubated in ABC-kit (prepared 30 minutes before adding to the slides, 1:800) for 2 hours at room temperature. After $2 \times 10$ minutes wash in TBS and $1 \times 10$ minutes wash in Tris-HCl ( $\mathrm{pH}$ 7.6), diaminobenzinidine (DAB) was added to the slides (filtrated DAB 1:1 in Tris- $\mathrm{HCl}$, supplemented with $10 \mu \mathrm{l} / 3 \mathrm{ml} \mathrm{H}_{2} \mathrm{O}_{2}$ right before use) and incubated for 10 minutes. The reaction was stopped by washing $3 \times 10$ minutes in TBS. Slides were dehydrated using an ethanol series ( $2 \times 5$ min ethanol 50\%, $2 \times 5$ min ethanol $70 \%, 3 \times 5$ min $2 \times 5$ min ethanol 95\%, 2×10 min ethanol pure, 2×10 min ultraclear) and subsequently embedded in DEPEX and coverslipped.

Synaptic changes were investigated by comparison of distribution of normal-sized and large-sized synaptophysin-immunoreactive presynaptic boutons (SIPBs) in the ipsilateral substantia gelatinosa (SG) compared with the contralateral SG. The contralateral SG was chosen as the control area because (1) internal control is the preferred option for immunohistochemical stainings due to variation between sections obtained from different animals, 
even if these sections are stained at the same moment [9], (2) detailed analysis (including von Frey test for mechanical allodynia, CatWalk gait analysis for sensory-motor function, and autotomy scores for auto-mutilation behavior) showed no indications of any behavioral changes in the hindpaw contralateral to the injured sciatic nerve [6]. Briefly, the ipsilateral (left) and contralateral (right) spinal cord dorsal horns were captured on a computer screen coupled to a computer-assisted Olympus AX-70 microscope. An average of 5 sections of each animal were photographed and analyzed. Transparent maps of the L3, L4, and L5 dorsal horn laminae (according to Molander and Grant [10]) were then matched to the captured dorsal horns on the computer screen. Three positions $x, y$, and $z$ within the SG of the ipsilateral and contralateral dorsal horn were put into focus using a $100 \times$ magnification and photomicrographs of these $x, y$, and $z$ positions were taken. Synaptophysin-immunoreactive presynaptic boutons (SIPBs) were quantified using the software Cell P® (Olympus Netherland B.V.). Due to methodological limitations, it was not possible to measure actual synaptic sizes. In order to study synaptic size changes according to Hebb's law, a previously described method was used [11]. Briefly, the mean area of 1,000 manually selected SIPBs was calculated within the contralateral SG. Here, we measured a mean SIPB size of $0.67 \mu \mathrm{m}^{2}$ with a standard deviation of $0.36 \mu \mathrm{m}^{2}$. The range of ((mean - SD); (mean + SD)) was considered as the category normal-sized SIPBS (i.e. 0.31 to $1.03 \mu \mathrm{m}^{2}$ ). SIPBs with an area below $0.31 \mu \mathrm{m}^{2}$ were considered non-specific and therefore excluded from analysis. Large-sized SIPBs were defined as SIPBs larger than (mean

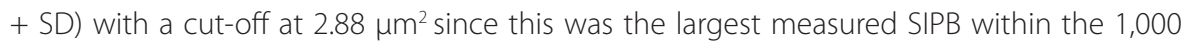
manually counted SIPBs. Hence, the range of large-sized SIPBs was from 1.03 to $2.88 \mu \mathrm{m}^{2}$. Normal-sized and large-sized SIPBs were selectively counted in those areas of the $x, y$, and $z$ positions which were in focus: the so-called regions of interest (ROI).

For statistical comparison between the ROl-area of the contralateral and ipsilateral SG and for the statistical comparison between the percent SIPBs within the ipsilateral and contralateral SG, a paired two-tailed Student-t-test was used. To investigate whether the mechanical allodynia observed was correlated with the number of large-sized SIPBs, a Pearson- $R$ correlation test was performed. A p-value below 0.05 was considered significant.

\section{Results}

All animals recovered well from surgery and showed immediate paresis of the affected left hindpaw, which persisted for the survival period of 84 days. At DPO 84, animals showed contractures of the ipsilateral hindpaw, further indicating the permanent nature of the lesion. Moreover, with proceeding time, all animals showed signs of autotomy. This means that the animals attacked the lateral aspects of the ipsilateral hindpaw, necessitating assessment of PWT to tactile stimulation using the medial-to-midplantar area of the hindpaw. The PWT of the ipsilateral hindpaw as assessed with von Frey filaments was significantly decreased at DPO 84 compared to baseline $(6.6 \pm 1.6 \mathrm{~g}$ and $58.8 \pm 11.4 \mathrm{~g}$, respectively; $p<0.01)$, whereas 
the PWT of the contralateral hindpaw was not statistically different between DPO 84 and baseline (39.8 $\pm 13.3 \mathrm{~g}$ and $43.3 \pm 13.5 \mathrm{~g}$, respectively; n.s.). Likewise, a significant difference was noted between the ipsilateral and contralateral PWT at DPO $84(p<0.05)$.

Then, normal-sized and large-sized SIPBs were counted within the ROls of $x, y$, and z positions within the SG of the L3-L5 spinal cord, both contralateral (fig. 1A) and ipsilateral (fig. 1B) to the nerve injury. The immunohistochemical staining of synaptophysin protein resulted in clusters of dark-coloured, pre-synaptic boutons, as shown in fig. 1. The ROI areas did not significantly differ between the ipsilateral and contralateral SGs $\left(1766 \pm 188 \mu \mathrm{m}^{2}\right.$ versus $1696 \pm 181 \mu \mathrm{m}^{2}$, respectively; n.s.), which allows for a valid comparison between the number of normal-sized and large-sized SIPBs. Quantitative analysis showed that normal-sized SIPBs did not significantly differ between the ipsilateral and contralateral SG (fig. 1C). However, in the large-sized category, SIPBs numbers were found to be elevated by $50.1 \%$ in the ipsilateral compared to the contralateral SG ( $p<0.05$; fig. 1C). Additional regression analysis showed that there was no statistical correlation between the ipsilateral PWT and the number of large-sized SIPBs $(p<0.05)$.
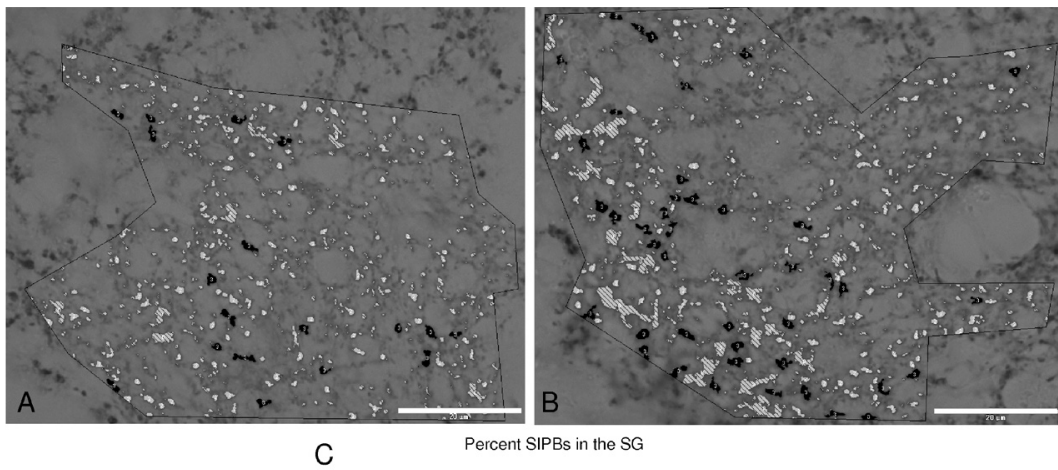

Percent SIPBs in the SG

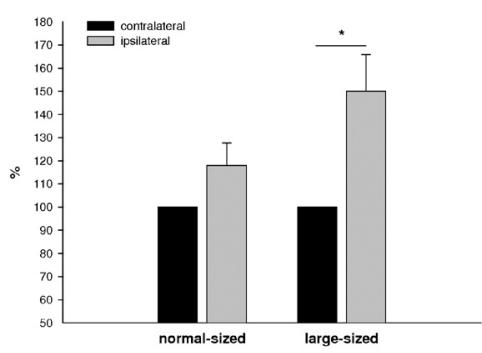

Figure 1. SIPBs were analyzed on the contralateral (A) and ipsilateral (B) dorsal horn. SIPBs were divided into normal-sized (in gray, numbered 2) and large-sized categories (in black, numbered 3). Striped boutons represent non-specific signal and are therefore excluded from the analysis. SIPBs numbers within the normal-sized and large-sized categories were quantified in the ipsilateral and contralateral SG (C). No significant differences were noted between ipsi-versus contralateral side for the normal-sized category, whereas the number of SIPBs of the large-sized category were significantly increased in the SG on the ipsilateral compared to the contralateral side. ${ }^{*}$ Student-T-test; $p<0,05$ 


\section{Discussion}

The present study shows that rats with chronic mechanical allodynia at 84 days after complete sciatic nerve injury have an increased number of large-sized SIPBs in the ipsilateral SG when compared to the contralateral SG. This finding may shed a different light on the neuropathological mechanisms thought to contribute to NP. Notably, the absence of a significant correlation between synaptic changes and pain outcome suggests that the level of synaptic change does not predict the degree of mechanical allodynia. Nevertheless, the present investigation points towards synaptic changes as potential contributors to NP at twelve weeks after PNI. Identification of the nature of the synaptic changes is necessary to understand the exact relation between the synaptic changes and chronic mechanical allodynia, and develop strategies to attenuate this pain condition. However, the nature of the synaptic changes was beyond the scope of the present investigation and therefore remains unknown at this stage. Nevertheless, the synaptic changes may relate to the chronic mechanical allodynia displayed by the animals, because the changes occurred within a crucial part of the central pain network, the substantia gelatinosa.

In the present study, we used a complete unilateral sciatic nerve injury model in order to induce chronic mechanical allodynia which persists for months [12]. This model is associated with the formation of neuroma-tissue at the site of PNI and this neuroma-tissue is a source of spontaneous and aberrant activity resulting in autotomy behavior $[5,13]$. Indeed, all animals in this study showed this autotomy behavior, which is also regarded a sign of spontaneous pain [5]. Spontaneous and aberrant activity from neuroma-tissue at the PNI site may have resulted in repetitive electrical input to the spinal cord dorsal horn [5]. According to Hebb's law, synaptic knobs enlarge due to repetitive firing of presynaptic cells [14]. The higher number of large-sized SIPBs observed in this study may therefore reflect an activity-dependent increase in synaptic strength and efficacy, ultimately resulting in the chronic mechanical allodynia observed in our neuropathic animals. Identification of the nature of the synaptic changes is necessary to understand the exact relation between the synaptic changes and chronic mechanical allodynia, and develop strategies to attenuate this pain condition.

The present data indicate that central plasticity may influence chronic NP, besides the already proven peripheral mechanisms of sensitization and plasticity. Upon cutting the sciatic nerve, the territories innervated by the sciatic nerve become deafferented and saphenous nerve endings, normally terminating in the medial part of the hindpaw, start sprouting into the denervated central area of the hindpaw [15]. This implies that nociceptive-stimulusinduced pain response of the hindpaw measured after complete sciatic nerve injury is mediated via saphenous nerve signaling. Importantly, the saphenous nerve mostly signals to the same segments of the spinal cord as the sciatic nerve (e.g. the L4 spinal cord segment) [16]. The synaptic changes induced by the continuous and spontaneous input from the sciatic nerve neuroma may, thus, also affect nociceptive signaling via the saphenous nerve, leading to chronic mechanical alloydnia, as observed in the present study. 


\section{Acknowledgements}

This work was financially supported by a Young Investigator Grant by the NVA (to R.D.), a grant from the Dutch Government (SENTERNOVEM \# IS 041064 to E.A.J.J., M.A.E.M. and R.D.), and two Kootstra fellowships of the Maastricht University (to R.J.P.J. and R.D.).

\section{References}

1. Nakamura, S. and R.R. Myers, Myelinated afferents sprout into lamina II of L3-5 dorsal horn following chronic constriction nerve injury in rats. Brain Res, 1999. 818(2): p. 285-90.

2. Shortland, P., E. Kinman, and C. Molander, Sprouting of A-fibre primary afferents into lamina II in two rat models of neuropathic pain. Eur J Pain, 1997. 1(3): p. 215-27.

3. Woolf, C.J., P. Shortland, and R.E. Coggeshall, Peripheral nerve injury triggers central sprouting of myelinated afferents. Nature, 1992. 355(6355): p. 75-8.

4. Chou, A.K., et al., Altered synaptophysin expression in the rat spinal cord after chronic constriction injury of sciatic nerve. Neurosci Lett, 2002. 333(3): p. 155-8.

5. Wall, P.D., et al., Autotomy following peripheral nerve lesions: experimental anaesthesia dolorosa. Pain, 1979. 7(2): p. 103-11.

6. Deumens, R., et al., The CatWalk gait analysis in assessment of both dynamic and static gait changes after adult rat sciatic nerve resection. J Neurosci Methods, 2007. 164(1): p. 120-30.

7. Schmalbruch, H., Fiber composition of the rat sciatic nerve. Anat Rec, 1986. 215(1): p. 71-81.

8. Smits, H., et al., Effect of spinal cord stimulation in an animal model of neuropathic pain relates to degree of tactile "allodynia". Neuroscience, 2006. 143(2): p. 541-6.

9. Hughes, D.I., et al., Peripheral axotomy induces depletion of the vesicular glutamate transporter VGLUT1 in central terminals of myelinated afferent fibres in the rat spinal cord. Brain Res, 2004. 1017(1-2): p. 69-76.

10. Molander, C., Grant, G., Spinal Cord Cytoarchitecture. Second ed. The Nervous System. 1995, San Diego: Academic Press.

11. Koopmans, G.C., et al., Circulating insulin-like growth factor I and functional recovery from spinal cord injury under enriched housing conditions. Eur J Neurosci, 2006. 23(4): p. 1035-46.

12. Dowdall, T., I. Robinson, and T.F. Meert, Comparison of five different rat models of peripheral nerve injury. Pharmacol Biochem Behav, 2005. 80(1): p. 93-108.

13. Seltzer, Z., Z.H. Rappaport, and D. Zagzag, A chronically implanted delivery system of drugs to a nerve-end neuroma: effects on a behavioural chronic pain model. J Neurosci Methods, 1985. 13(3-4): p. 223-9.

14. Hebb, D.O., The organization of Behavior. 1949, New York: Wiley.

15. Kingery, W.S. and J.A. Vallin, The development of chronic mechanical hyperalgesia, autotomy and collateral sprouting following sciatic nerve section in rat. Pain, 1989. 38(3): p. 321-32.

16. Markus, H. and B. Pomeranz, Saphenous has weak ineffective synapses in sciatic territory of rat spinal cord: electrical stimulation of the saphenous or application of drugs reveal these somatotopically inappropriate synapses. Brain Res, 1987. 416(2): p. 315-21. 


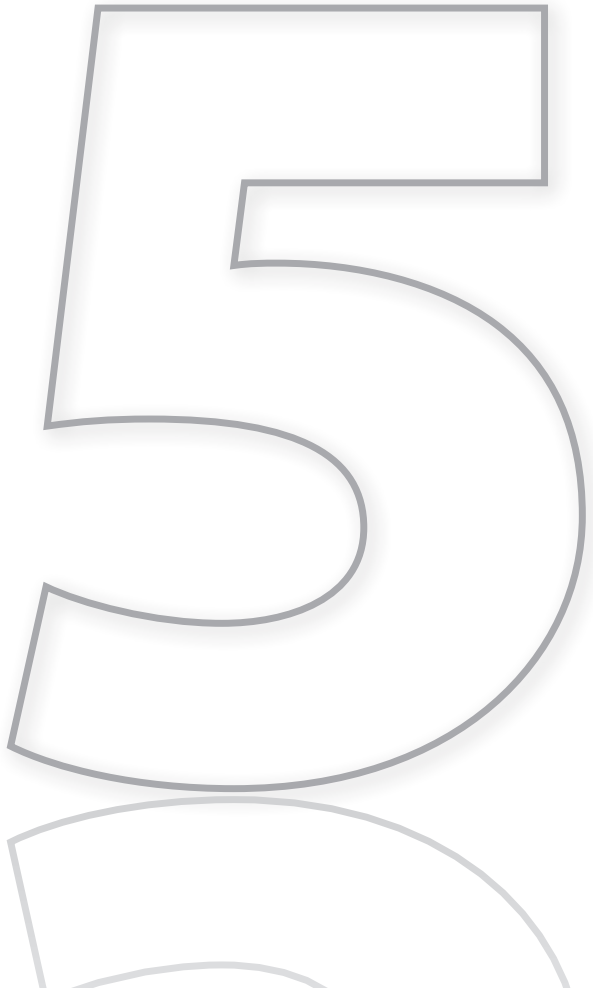

Inverse relation between intensity of GFAP expression in the substantia gelatinosa and degree of chronic mechanical allodynia

R. Deumens, R.J.P. Jaken, L. Knaepen, I. Van der Meulen, E.A.J. Joosten 


\section{Abstract}

Glial cells are known to have a large impact on neuropathic pain conditions. Within the spinal cord, microglia rapidly respond to peripheral nerve injury, resulting in central sensitization and ultimately in onset of enhanced pain behavior. Astroglia respond with a short delay and are thought to contribute to the early maintenance of neuropathic pain. Nevertheless, it is unknown whether the roles of these glial cell types can be influenced by the chronicity of the neuropathology. Here, the persistent responses of astroglia and microglia to peripheral nerve injury within central pain networks in the upper dorsal horn laminae were studied. At twelve weeks after complete sciatic nerve injury, upregulation of glial fibrillary acidic protein (GFAP), but not complement receptor 3, could be detected in laminae II and III. Moreover, it was found that neuropathic animals with a higher degree of mechanical allodynia had a lower intensity of GFAP expression in lamina II (substantia gelatinosa). From these data we conclude that the role of astroglial responses in mechanical allodynia after peripheral nerve injury may be less straightforward as previously thought. Although astroglia are known to play a pronociceptive role in early neuropathic pain states, this role may shift to anti-nociception in more chronic pain states. 


\section{Introduction}

Neuropathic pain is mostly resistant to currently available pain medication [1]. This fact may be causally related to incomplete knowledge about mechanisms underlying neuropathic pain. For a long time a neuron-centric view on neuropathic pain has prevailed, but data obtained in the last decade strongly suggest an important role of non-neuronal elements in the onset and maintenance of neuropathic pain [2-5]. A special role has been proposed for astroglia and microglia, which are responsible for immune responses in the immune-privileged central nervous [6]. Upon peripheral nerve injury (PNI) these glia become reactive in both spinal $[7,8]$ and supraspinal locations [9]. It is now understood that the onset of neuropathic pain is strongly dependent on PNI-induced activation of microglia [10-13]. However, microglial activation does not seem to play a vital role in the maintenance of neuropathic pain [14].

The microglial response to PNI is followed by an astroglial response [15-17]. The latter can persist for prolonged periods, even up to three months after injury $[18,19]$. This response is classically detectable by an upregulation of glial fibrillary acidic protein (GFAP) as a result of hypertrophied rather than proliferating astroglia [18]. Within 10 days after complete sciatic nerve injury, the upregulation of GFAP in the ipsilateral grey matter was found to positively correlate with the degree of mechanical allodynia [20]. Moreover, suppression of the astroglial response up till three weeks after injury can partially reverse PNI-induced mechanical allodynia [21-23].

Although glial response to PNI within the first three weeks is known to contribute to pain, it is unknown if and how the persistent glial responses lasting up to three months [19] influence these more chronic pain states. In the present study, the microglial and astroglial responses to PNI were studied in the superficial laminae (I-III) of the lumbar dorsal horn. Complete injury of the sciatic nerve was used as this PNI model is associated with clear chronic neuropathic pain conditions [24]. A specific activation of astroglia was detected in laminae II and III and, interestingly, neuropathic rats with a higher degree of astroglial activation were found to be less allodynic to tactile stimulation of the hind paw. The present data may, therefore, shed a new light on the current opinion, regarding glia as cells solely exacerbating neuropathic pain conditions.

\section{Materials and methods}

All experimental procedures were carried out according to the recommendations of the European Commission (European Communities Council Directive of 24 November 1986; 86/609/EEC) and protocols were approved by the Committee on Animal Research of the Maastricht University (DEC 2006-054). Every effort was made to minimize the number of animals and their pain and to maximise their comfort. Nine male Wistar rats (Charles River, The 
Netherlands) at the age of 9 weeks were used in this study. Rats were separately housed in a temperature $\left(19-24^{\circ} \mathrm{C}\right)$ and humidity $(55 \pm 15 \%)$ conditioned room with a normal $12: 12 \mathrm{~h}$ light/dark regime and background music to calm the animals.

Baseline values of paw withdrawal thresholds (PWT) to tactile stimuli were obtained within the first week before surgery as previously described [25]. Importantly, von Frey filaments (North Coast Medical Inc. Morgan Hill, CA, USA) were applied to the medialto-midplantar surface of the hind paws (which corresponds to the innervation territory of the saphenous nerve [26]) and a positive withdrawal responses was only considered when the animal displayed active attention to the stimulus (such as clear postural shifts, licking the paw, or attacking the filament). PWT of both hind paws were determined (the right hind paw served as the control paw).

Complete unilateral sciatic nerve injury was performed as previously described [27]. In this model, the sciatic nerve was completely transected and a $1 \mathrm{~cm}$ long piece of nerve was resected to prevent regeneration. By using this procedure, a chronic neuropathology of the sciatic nerve is modeled, which does not allow for any regeneration or functional recovery [27]. The contralateral paw was left intact and served as the control side.

During a post-operative period of twelve weeks animals were monitored for autotomy behavior, which is regarded a sign of spontaneous pain [28] and PWT to tactile stimuli were determined. Then, animals were sacrificed by an intraperitoneal injection of Nembutal (150 mg/kg body weight) followed by transcardial perfusion with salt solution to remove blood was followed by perfusion with ice-cold 4\% paraformaldehyde (PF) in $0.1 \mathrm{M}$ phosphate buffer ( $\mathrm{pH}$ 7.4). After perfusion, the lumbar spinal cord was post-fixed overnight in cold 4\% buffered PF. Then, cryoprotection was performed by an overnight incubation in $10 \%$ sucrose solution (in $0.1 \mathrm{M}$ phosphate buffer; $\mathrm{pH} 7.6$; at $4^{\circ} \mathrm{C}$ ) and a three-day-incubation in $25 \%$ sucrose solution. Subsequently, the lumbar spinal cord (lumbar levels L3-L6) were frozen and stored at $-80^{\circ} \mathrm{C}$ until cryostate sectioning. The spinal cords $(n=9)$ were transversally sectioned on a Leica cryostat to obtain $25 \mu$ m-sections of levels L3-L6 at which the sciatic nerve enters the spinal cord. Sections were carefully mounted on gelatin chrome-alumn (Sigma, Uithoorn, The Netherlands) coated glass slides and stored at $-20^{\circ} \mathrm{C}$ until processing for histological staining.

After thawing the glass slides, immunostaining was performed to stain every tenth section for complement receptor-3 (CR3; for microglia) or glial fibrillary acidic protein (GFAP; for astroglia). For CR3 staining, sections were washed in three consecutive steps with phosphate-buffered-saline (PBS) and then blocked for 30 min using blocking solution (PBS containing 5\% normal donkey serum, 2\% BSA, and 0.1\% Triton-X100). This was followed by an overnight incubation in primary antibody solution (mouse anti-CD11b/c; OX-42; BD Biosciences; 1:250 in blocking solution). The next day, sections were washed again (3 times 10 min PBS) and incubated in secondary antibody solution (Cy-3 conjugated donkey anti-mouse; Sanbio; diluted 1:800 in PBS) for one hour and a half. This was followed by three washing steps (10 min PBS each) and embedding the sections in 80\% glycerol/PBS and coverslipping. 
For GFAP staining, the procedure was similar with the exception of different wash-buffers: tris-buffered-saline (TBS) with 0.3\% triton-X100 (TBS-T), and a different primary antibody solution (rabbit anti-GFAP; DAKO; diluted 1:1600 in TBS-T). After the GFAP staining procedure, slides were embedded in 80\% glycerol/TBS and coverslipped.

Photomicrographs of ipsilateral and contralateral dorsal horns were taken using a fluorescent microscope (Olympus Ax70) with a 10x objective (and a 12.5× condenser) and a grayscale F-view camera. Per animal about 40 photomicrographs were collected and analyzed using CellPœ software. Importantly, laminae I, II, and III were delineated according to a rat spinal cord atlas [29]. Transparent schematic drawings were made according to the structure of the spinal cord as represented in this atlas. Placement of these drawings onto the photomicrographs was done to delineate laminae I to $\mathrm{VI}$ as regions of interest (ROI). The mean grey value of GFAP or CR3 stained sections was measured in each individual lamina. Laminae I-III on the contralateral side of the spinal cord served as the control.

For statistical analysis, analysis of variance (ANOVA) with repeated measures over time (with Bonferroni post-hoc correction) was used to detect changes in the ipsilateral and contralateral PWT to tactile stimuli. The ipsilateral PWT and the contralateral PWT at individual time points were compared using a paired Student's t-test. Also, the mean grey (GFAP intensity) values of the ipsilateral and contralateral laminae were compared using a paired Student's t-test. Finally, correlations between ipsilateral PWT at 12 weeks after complete sciatic nerve injury and mean grey (GFAP intensity) values in individual laminae (I-III) were assessed using a Pearson-R correlation test. A p-value of 0.05 was regarded as the level of statistical significance.

\section{Results}

All animals recovered well from surgery. In addition to a paresis of the nerve-injured left hind paw, all animals attacked the outer parts of their left hind paw (i.e. autotomy behavior), confirming previous observations in this same model $[27,28]$. Because of this autotomy behavior, PWT were determined after mechanical stimulation of the medial-to-midplantar hindpaw. This region of the hindpaw is important since it is innervated by the spared saphenous nerve and PWT can be obtained even in the absence of a sciatic innervation. Importantly, at twelve weeks after injury, animals were found to have severe contractures of the ankle joint, reflecting the permanent nature of the sciatic nerve injury.

At a survival time of twelve weeks after injury, astroglia and microglia in the L3-L6 spinal cord were stained for GFAP and CR3, respectively (Figure 1A-B). The astroglial response, as represented by a higher intensity (grey value) of the GFAP immunoreactivity, appeared to occur in the dorsal horn ipsilateral to the injured nerve (Figure 1A"). Microglia were found to display a 'resting phenotype' with long slender processes (Figure 1B'). Quantification of the GFAP and CR3 immunoreactivity was performed in individual laminae I-III (Figure 1C). A small but significant upregulation of the GFAP intensity was found only within 
the ipsilateral laminae II and III as compared to the contralateral side (Figure 1D). No difference was found between the intensity of CR3 immunostaining between the ipsilateral and contralateral laminae (Figure 1E).

The sciatic nerve injury resulted in a dramatic and significant drop of the ipsilateral PWT (ANOVA with repeated measures over time: F6,8 $=16.2, p<0.001$; Figure 1F). From baseline values of $55.1 \pm 10.7$ grams, the PWT of the ipsilateral hind paw decreased to values between $15.3 \pm 2.2$ grams at 2 weeks and $4.4 \pm 0.7$ grams at 6 weeks after injury. At 12 weeks after injury, PWT of the ipsilateral hind paw was still significantly decreased (value: $6.3 \pm 1.4$ grams) as compared to baseline values $(p<0.05)$. On the contrary, the PWT of the contralateral (right) hind paw was unaffected by the surgical intervention (ANOVA with repeated measures over time: $F 6,8=1.1, n$.s.). Correlation tests were performed to determine whether the degree of GFAP immunoreactivity was linked to the degree of ipsilateral PWT at twelve weeks after injury. Indeed a correlation was found, but only between the ipsilateral PWT and the ipsilateral astroglial activation in lamina II (Figure 1G-H). Importantly, the highly significant correlation was found to be positive, which means that animals with a higher degree of GFAP immunoreactivity in lamina II have a lower degree of mechanical allodynia than animals with less GFAP immunoreactivity in lamina II.

\section{Discussion}

There is a strong interest in elucidating the role of glial cells in the onset and/or maintenance of neuropathic pain. Peripheral neuropathies are known to induce an inflammatory response which extends to the central nervous system and both microglia and astroglia are thought to have a pronounced role in the neuropathic pain conditions which ensue [6,30]. Nevertheless, the role of these two glial cell types is quite different in nature as data indicate that microglia are of vital importance to onset of neuropathic pain, whereas astroglia are associated with maintenance of neuropathic pain within the first three weeks after PNI [14,30]. In the present study, we focused on PNI-induced glial responses in the superficial laminae of the lumbar spinal cord at three months after peripheral neuropathy. We found that a persistent upregulation of GFAP occurred in lamina II and III, but that neuropathic animals with a higher intensity of GFAP immunoreactivity in lamina II had a lower degree of mechanical allodynia.

PNI-induced glial responses are known to occur for prolonged periods, even up to three months [19]. Microglia rapidly respond to PNI by hypertrophy of the cell soma and retraction of their processes, which are normally used to screen the microenvironment during immune-surveillance. This morphological change of microglia is paralleled by a change in the molecular profile of these cells, and especially the enhanced expression of purinoceptors and toll-like receptors has been shown crucial in the onset of neuropathic pain conditions [13,31-33]. Nevertheless, reactive microglia do not seem to contribute to already established mechanical allodynia [14]. Microglial responses to PNI are followed by upregulation of GFAP 


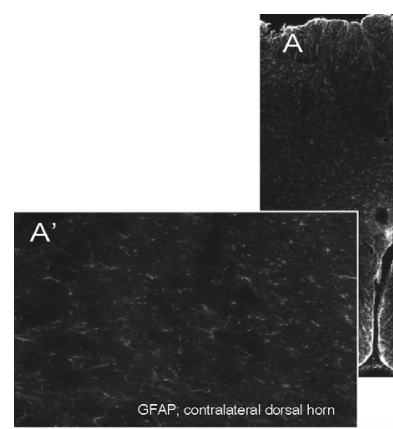

C

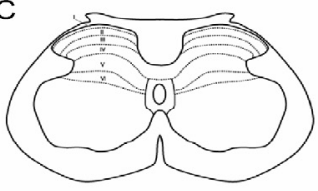

F Paw Withdrawal Threshold

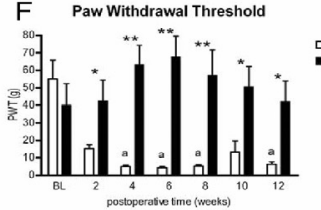

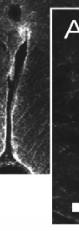

D

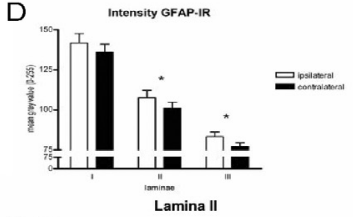

G

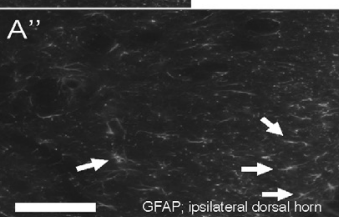

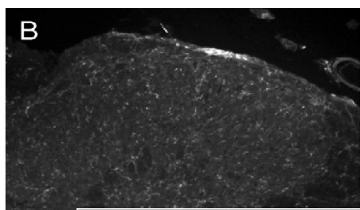

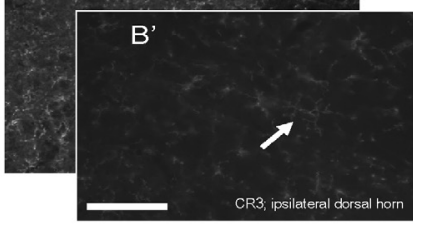

E

E imtensily Cr3-18
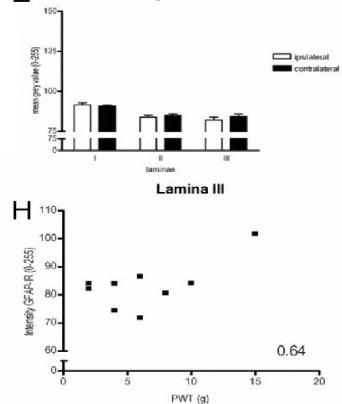

Figure 1. Glial responses and mechanical allodynia at 12 weeks after complete unilateral sciatic nerve injury. GFAP (A) and CR3 ( $B$; showing ipsilateral dorsal horn) immunostaining of the lumbar spinal cord at 12 weeks after sciatic nerve injury. The arrows in $\mathrm{A}^{\prime \prime}$ indicate hypertrophic astroglia, residing in lamina II near the border with lamina III. The arrow in $\mathrm{B}^{\prime}$ indicates a microglial cell with a typical 'resting' phenotype. Dorsal horns were divided in six laminae (C), and the intensity of GFAP (D) and CR3 (E) immunostaining was measured in lamina I-III (*, $p<0.05)$. Paw withdrawal thresholds of the ipsilateral, but not contralateral hindpaw were decreased compared to baseline $(F){ }^{*}, p<0.05$ as compared to the contralateral PWT; **, $p<0.01$ as compared to the contralateral PWT; $a, p<0.05$ as compared to the PWT at baseline (BL). Correlation analysis (GH) showed that GFAP immunoreactivity in lamina II was positively correlated with the ipsilateral PWT. (**, $p<0.01)$. PWT, paw withdrawal threshold; GFAP, glial fibrillary acidic protein; CR3, complement receptor-3; IR, immunoreactivity. Scale bars represent $100 \mu \mathrm{m}$. 
expression, indicative of astroglial responses [15,34]. This astroglial response, which may be elicited by the early microglial responses [34, 35], occurs with a change in its molecular phenotype. Pharmacological attenuation of a range of molecules upregulated in reactive astroglia upon PNI has been shown to reverse mechanical allodynia up to three weeks after injury $[21,22,36]$. However, the role of the PNI-induced astroglial response in neuropathic pain conditions beyond three weeks after injury is unknown, as are the molecular phenotypes of astroglia at such chronic periods. We here selected a complete rat sciatic nerve injury model, known to elicit signs of neuropathic pain which last for several months [24] and focused exclusively on the glial responses at 12 weeks after injury. Importantly, this model does not show mirror pain, a phenomenon observed in other pain models such as sciatic inflammatory neuropathy and partial sciatic nerve ligation [37,38]. Moreover, astroglia are reported to specifically upregulate GFAP ipsilateral to a sciatic nerve transection [18]. The specific neuropathic pain and astroglial activation response ipsilateral to sciatic nerve transection allows for the use of the contralateral side as an internal control. In the present investigation, small increases in GFAP, but not CR3 expression were detected in lamina II and III of the lumbar dorsal horn. Interestingly, laminae II is strongly involved in nociceptive signal processing. The upregulation of GFAP within this lamina, therefore, suggests a relation between the primary afferent fibres and activated astroglia. Indeed, upregulation of GFAP can be triggered by activity of primary afferents under both non-pathological and nerve injury conditions [7]. We speculate that the persistent increase in GFAP expression at twelve weeks is related to chronic afferent input to the dorsal horn, because all animals in our model displayed autotomy, which is suggested to result from ectopic activity of injured primary afferent fibers after sciatic nerve injury [28]. An alternative explanation for enhanced GFAP expression is selective neuronal cell death in the superficial dorsal horn induced by afferent fiber activation in the sectioned sciatic nerve [39].

Our data do not imply a cause-effect ratio between GFAP expression and allodynic responses. Nevertheless, the negative correlation between GFAP expression in the ipsilateral substantia gelatinosa and the ipsilateral PWT is suggestive of an anti-nociceptive role of the astroglia at twelve weeks after sciatic nerve injury. Although this finding may have important implications on the view of glial responses to PNI and the link to neuropathic pain, future investigations ought to demonstrate the molecular profile of responsive astroglia at prolonged time points after PNI. From there, astroglial responses can be interfered with at the molecular level in order to demonstrate the causal link between the molecular profile of reactive astroglia and chronic neuropathic pain behavior. However, it is already known that astroglia do not only express molecular cues which may potentiate neuronal signalling. Astroglia in culture have been reported to express endocannabinoids, such as anandamide and 2-arachinonolyl glycerol (2-AG) $[40,41]$. Nociceptors and spinal interneurons are known to express cannabinoid CB1 receptors [42,43]. Importantly, cannabinoid CB1 receptor activation leads to inhibition of the transmission of C- and A $\delta$-fiber evoked responses [44]. Although the cannabinoid system plays an important role in attenuation of nociceptive 
signalling [45] and endocannabinoids can be expressed by astroglia, it remains to be determined whether such or other molecular processes linked to nociception occur in the persistent astroglial response to PNI.

The present data suggest that the morphological change in astroglia, as detected by increased GFAP expression, does not predict the nature of astroglial interaction with the environment. Although the reactive astroglial morphology may be similar at early and late stages after nerve injury, the effect of reactive astroglia on neuropathic pain may be completely different as a result of context and time. Indeed, a recent investigation showed that transplantation of stem cells into injured spinal cords triggered mechanical allodynia when these cells differentiated into astroglia [46]. Also, transplantation of immature astroglia treated with ciliary neurotrophic factor resulted in mechanical allodynia in spinally injured rats [47]. However, when immature astroglia were treated with bone morphogenetic protein-4 before transplantation into the injured spinal cord, animals did not develop mechanical allodynia [47]. These data, thus, show that the molecular cues to which astroglia are exposed may determine the relationship between the astroglia and neuropathic pain. Therefore, we suggest that future studies ought to address the link between astroglia at different stages after PNI and neuropathic pain.

In conclusion, we demonstrated that enhanced GFAP expression can be detected up to twelve weeks after complete sciatic nerve injury, specifically in lamina II and III of the dorsal horn. Neuropathic rats with a higher intensity of GFAP expression in the substantia gelatinosa were found to display less severe mechanical allodynia. These data may shed a different light on the current view on astroglia as pro-nociceptive cells.

\section{Acknowledgements}

The authors are grateful to Dr Jaap Patijn (Department of Anesthesiology, Academic Hospital Maastricht; neurologist) for critically reading the manuscript. This work was financially supported by a grant from the Dutch Polymer Institute (DPI grant \# 608 to E.A.J.J., M.A.E.M. and R.D.), a Young Investigator Grant by the NVA (to R.D.), a grant from the Dutch Government (SENTERNOVEM grant \# IS 041064; to E.A.J.J. and M.A.E.M.), and two Kootstra fellowships of the Maastricht University (to R.J.P.J. and R.D.).

\section{References}

1. McCleane, G., Pharmacological management of neuropathic pain. CNS Drugs, 2003. 17(14): p. 1031-43.

2. Watkins, L.R., E.D. Milligan, and S.F. Maier, Glial activation: a driving force for pathological pain. Trends Neurosci, 2001. 24(8): p. 450-5. 
3. Watkins, L.R. and S.F. Maier, Beyond neurons: evidence that immune and glial cells contribute to pathological pain states. Physiol Rev, 2002. 82(4): p. 981-1011.

4. Watkins, L.R. and S.F. Maier, Glia: a novel drug discovery target for clinical pain. Nat Rev Drug Discov, 2003. 2(12): p. 973-85.

5. Scholz, J. and C.J. Woolf, The neuropathic pain triad: neurons, immune cells and glia. Nat Neurosci, 2007. 10(11): p. 1361-8.

6. Marchand, F., M. Perretti, and S.B. McMahon, Role of the immune system in chronic pain. Nat Rev Neurosci, 2005. 6(7): p. 521-32.

7. Garrison, C.J., P.M. Dougherty, and S.M. Carlton, GFAP expression in lumbar spinal cord of naive and neuropathic rats treated with MK-801. Exp Neurol, 1994. 129(2): p. 237-43.

8. Colburn, R.W., A.J. Rickman, and J.A. DeLeo, The effect of site and type of nerve injury on spinal glial activation and neuropathic pain behavior. Exp Neurol, 1999. 157(2): p. 289-304.

9. Kuzumaki, N., et al., Chronic pain-induced astrocyte activation in the cingulate cortex with no change in neural or glial differentiation from neural stem cells in mice. Neurosci Lett, 2007. 415(1): p. 22-7.

10. Jin, S.X., et al., p38 mitogen-activated protein kinase is activated after a spinal nerve ligation in spinal cord microglia and dorsal root ganglion neurons and contributes to the generation of neuropathic pain. J Neurosci, 2003. 23(10): p. 4017-22.

11. Coull, J.A., et al., BDNF from microglia causes the shift in neuronal anion gradient underlying neuropathic pain. Nature, 2005. 438(7070): p. 1017-21.

12. Tsuda, M., K. Inoue, and M.W. Salter, Neuropathic pain and spinal microglia: a big problem from molecules in "small" glia. Trends Neurosci, 2005. 28(2): p. 101-7.

13. Tozaki-Saitoh, H., et al., P2Y12 receptors in spinal microglia are required for neuropathic pain after peripheral nerve injury. J Neurosci, 2008. 28(19): p. 4949-56.

14. Raghavendra, V., F. Tanga, and J.A. DeLeo, Inhibition of microglial activation attenuates the development but not existing hypersensitivity in a rat model of neuropathy. J Pharmacol Exp Ther, 2003. 306(2): p. $624-30$

15. Zhang, J. and Y. De Koninck, Spatial and temporal relationship between monocyte chemoattractant protein-1 expression and spinal glial activation following peripheral nerve injury. J Neurochem, 2006. 97(3): p. 772-83.

16. Ji, R.R., et al., Possible role of spinal astrocytes in maintaining chronic pain sensitization: review of current evidence with focus on bFGF/JNK pathway. Neuron Glia Biol, 2006. 2(4): p. 259-269.

17. Romero-Sandoval, A., et al., A comparison of spinal lbal and GFAP expression in rodent models of acute and chronic pain. Brain Res, 2008. 1219: p. 116-26.

18. Murray, M., et al., Modification of astrocytes in the spinal cord following dorsal root or peripheral nerve lesions. Exp Neurol, 1990. 110(3): p. 248-57.

19. Coyle, D.E., Partial peripheral nerve injury leads to activation of astroglia and microglia which parallels the development of allodynic behavior. Glia, 1998. 23(1): p. 75-83.

20. Garrison, C.J., et al., Staining of glial fibrillary acidic protein (GFAP) in lumbar spinal cord increases following a sciatic nerve constriction injury. Brain Res, 1991. 565(1): p. 1-7. 
21. Zhuang, Z.Y., et al., ERK is sequentially activated in neurons, microglia, and astrocytes by spinal nerve ligation and contributes to mechanical allodynia in this neuropathic pain model. Pain, 2005. 114(1-2): p. 149-59.

22. Zhuang, Z.Y., et al., A peptide c-Jun N-terminal kinase (JNK) inhibitor blocks mechanical allodynia after spinal nerve ligation: respective roles of JNK activation in primary sensory neurons and spinal astrocytes for neuropathic pain development and maintenance. J Neurosci, 2006. 26(13): p. 3551-60.

23. Katsura, H., et al., Transforming growth factor-activated kinase 1 induced in spinal astrocytes contributes to mechanical hypersensitivity after nerve injury. Glia, 2008. 56(7): p. 723-33.

24. Dowdall, T., I. Robinson, and T.F. Meert, Comparison of five different rat models of peripheral nerve injury. Pharmacol Biochem Behav, 2005. 80(1): p. 93-108.

25. Smits, H., et al., Effect of spinal cord stimulation in an animal model of neuropathic pain relates to degree of tactile "allodynia". Neuroscience, 2006. 143(2): p. 541-6.

26. Decosterd, I. and C.J. Woolf, Spared nerve injury: an animal model of persistent peripheral neuropathic pain. Pain, 2000. 87(2): p. 149-58.

27. Deumens, R., et al., The CatWalk gait analysis in assessment of both dynamic and static gait changes after adult rat sciatic nerve resection. J Neurosci Methods, 2007. 164(1): p. 120-30.

28. Wall, P.D., et al., Autotomy following peripheral nerve lesions: experimental anaesthesia dolorosa. Pain, 1979. 7(2): p. 103-11.

29. Molander, C. and G. Grant, Spinal cord cytoarchitecture. The Nervous System, 1995. 2nd Edition(Academic Press): p. San Diego.

30. McMahon, S.B., W.B. Cafferty, and F. Marchand, Immune and glial cell factors as pain mediators and modulators. Exp Neurol, 2005. 192(2): p. 444-62.

31. Tsuda, M., et al., P2X4 receptors induced in spinal microglia gate tactile allodynia after nerve injury. Nature, 2003. 424(6950): p. 778-83.

32. Obata, K., et al., Toll-like receptor 3 contributes to spinal glial activation and tactile allodynia after nerve injury. J Neurochem, 2008.

33. Bettoni, I., et al., Glial TLR4 receptor as new target to treat neuropathic pain: efficacy of a new receptor antagonist in a model of peripheral nerve injury in mice. Glia, 2008. 56(12): p. 1312-9.

34. Tanga, F.Y., V. Raghavendra, and J.A. DeLeo, Quantitative real-time RT-PCR assessment of spinal microglial and astrocytic activation markers in a rat model of neuropathic pain. Neurochem Int, 2004. 45(2-3): p. 397-407.

35. Tilleux, S., J. Berger, and E. Hermans, Induction of astrogliosis by activated microglia is associated with a down-regulation of metabotropic glutamate receptor 5. J Neuroimmunol, 2007. 189(1-2): p. 23-30.

36. Kozai, T., et al., Tissue type plasminogen activator induced in rat dorsal horn astrocytes contributes to mechanical hypersensitivity following dorsal root injury. Glia, 2007. 55(6): p. 595-603.

37. Seltzer, Z., R. Dubner, and Y. Shir, A novel behavioral model of neuropathic pain disorders produced in rats by partial sciatic nerve injury. Pain, 1990. 43(2): p. 205-18.

38. Milligan, E.D., et al., Spinal glia and proinflammatory cytokines mediate mirror-image neuropathic pain in rats. J Neurosci, 2003. 23(3): p. 1026-40. 
39. Coggeshall, R.E., et al., A-fiber sensory input induces neuronal cell death in the dorsal horn of the adult rat spinal cord. J Comp Neurol, 2001. 435(3): p. 276-82.

40. Walter, L., et al., Astrocytes in culture produce anandamide and other acylethanolamides. J Biol Chem, 2002. 277(23): p. 20869-76.

41. Walter, L. and N. Stella, Endothelin-1 increases 2-arachidonoyl glycerol (2-AG) production in astrocytes. Glia, 2003. 44(1): p. 85-90.

42. Farquhar-Smith, W.P., et al., Cannabinoid CB(1) receptor expression in rat spinal cord. Mol Cell Neurosci, 2000. 15(6): p. 510-21.

43. Salio, C., et al., Pre-and postsynaptic localizations of the CB1 cannabinoid receptor in the dorsal horn of the rat spinal cord. Neuroscience, 2002. 110(4): p. 755-64.

44. Kelly, S. and V. Chapman, Selective cannabinoid CB1 receptor activation inhibits spinal nociceptive transmission in vivo. J Neurophysiol, 2001. 86(6): p. 3061-4.

45. Hohmann, A.G. and R.L. Suplita, 2nd, Endocannabinoid mechanisms of pain modulation. Aaps J, 2006. 8(4): p. E693-708.

46. Hofstetter, C.P., et al., Allodynia limits the usefulness of intraspinal neural stem cell grafts; directed differentiation improves outcome. Nat Neurosci, 2005. 8(3): p. 346-53.

47. Davies, J.E., et al., Transplanted astrocytes derived from BMP- or CNTF-treated glial-restricted precursors have opposite effects on recovery and allodynia after spinal cord injury. J Biol, 2008. 7(7): p. 24. 


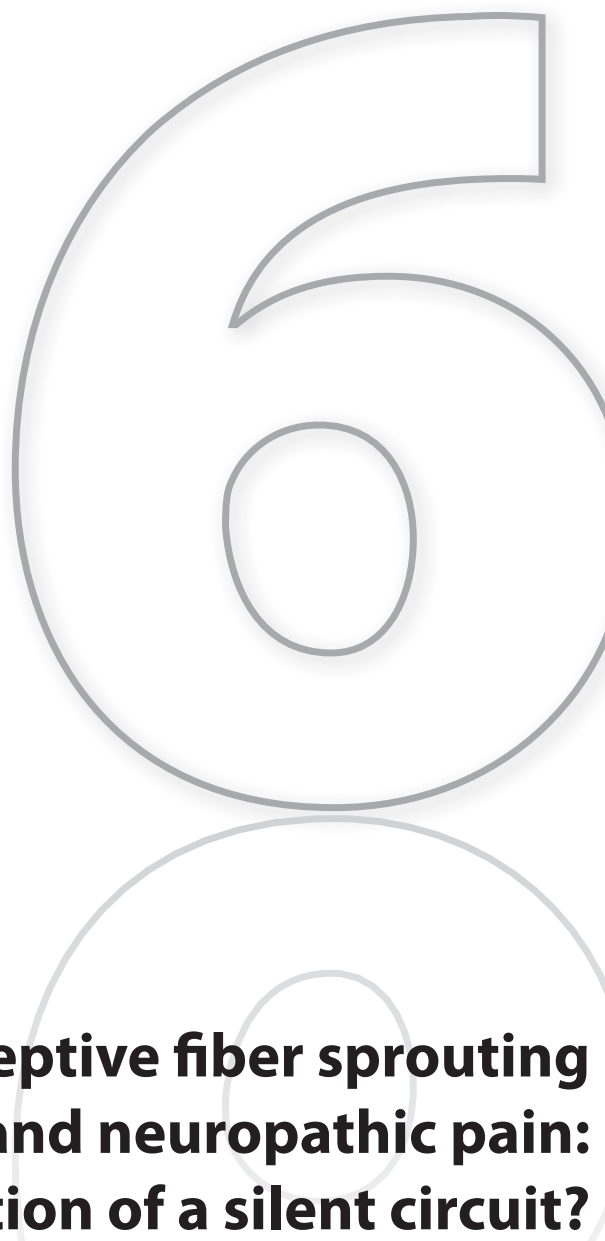

\section{Nociceptive fiber sprouting and neuropathic pain: activation of a silent circuit?}

R.J.P. Jaken, S.F.J. van Gorp, E.A.J. Joosten, P. Martínez- Martínez, M. Losen, M.H. de Baets, M.A.E. Marcus, R. Deumens 


\section{Abstract}

Structural changes within the spinal nociceptive network may be fundamental to both the chronic and the intractable nature of neuropathic pain. Interneurons expressing the $\gamma$-isoform of protein kinase C $(P K C \gamma)$, being part of the nociceptive network and specifically receiving $A \beta$ fiber input, are pivotal in the development of mechanical allodynia following peripheral nerve injury. Here, we hypothesized that nerve injury resulting in glial activation is involved in fiber sprouting within and above the spinal PKCY layer, thereby activating a normally silent circuit between $A \beta$ fibers and nociceptionspecific projection neurons located in the superficial dorsal horn. Non-nociceptive hindpaw stimulation in $L 5$ spinal nerve transected rats was found to strongly induce a marker of early neuronal activation, c-Fos, in both the spinal PKCY layer and more superficial layers ipsilateral, but not contralateral to nerve injury. A pronounced increase in growth associated protein-43 (GAP-43) immunoreactivity was observed within these ipsilateral spinal layers from 5 till 10 days post-injury. GAP-43 was largely colocalized with calcitonin-gene-relatedprotein suggesting the nociceptive nature of these sprouting fibers. Daily intrathecal injections of propentofylline for 5 days attenuated mechanical allodynia and microglial activation in the spinal dorsal horn, but did not affect GAP-43 levels. Our findings suggest that sprouting of nociceptive fibers following nerve injury results in gating of $A \beta$ fiber input to more superficial layers containing nociception-specific projection neurons. The structural changes as observed within the crucial part of the spinal nociceptive network may explain the chronic nature of neuropathic pain and the ineffectiveness of current anti-pain medicine. 


\section{Introduction}

The spinal dorsal horn is of vital importance for the integration and further processing of nociceptive and non-nociceptive information derived from primary afferent fibers (A $\delta / C$ fibers and $A \beta$ fibers, respectively). These distinct fibers have their specific termination patterns within the spinal dorsal horn, with $A \delta$ and $C$ fibers terminating in the most superficial layers (including lamina I to the most dorsal part of inner lamina $I_{;} I-\|_{i d}$ ) and A $\beta$ fibers in deeper layers (including the most ventral part of inner lamina II to lamina VI; $\|_{i v}-V I$ ) [1]. Importantly, $A \beta$ fibers activate a subset of supposedly excitatory interneurons expressing protein kinase C gamma (PKCY) [2], which were shown to be of vital importance in the development of mechanical allodynia following nerve injury [3]. These PKCY positive interneurons are thought to be part of a normally inactive circuit, which, if activated, gate information from non-nociceptive fibers to nociception-specific (NS) projection neurons in lamina I, thereby contributing to mechanical allodynia $[4,5]$. Activation of this circuit may be mediated via enhanced primary afferent input. Primary afferents, which include non-nociceptive and nociceptive fibers, have been reported to sprout following nerve injury, a process which has been suggested to contribute to injury-induced mechanical allodynia [6-9].

Fiber sprouting depends on the availability of growth factors, and it is known that peptidergic C fibers sprout in response to increased levels of nerve growth factor (NGF) [10], while neurotrophin-3 (NT-3) triggers sprouting of A $\beta$ afferents [1 1]. Glial-cell line derived neurotrophic factor (GDNF) may exert growth-promoting effects on non-peptidergic C fibers via their specific expression of GDNF family receptor a1 [12,13]. Well-known sources of these growth factors include microglia and astroglia $[14,15,16,17]$, which show robust activation following peripheral nerve injury [18]. Importantly, inhibition of glial activation strongly attenuates nerve-injury-induced symptoms of neuropathic pain including mechanical allodynia $[19,20]$. In the present study, we hypothesized that nerve-injury-induced fiber sprouting within and above the spinal PKC $y$ layer triggers the activation of a silent circuit between $A \beta$ fibers innervating the spinal PKC $Y$ layer and nociception-specific projection neurons in the more superficial dorsal horn. We furthermore studied the involvement of glial activation in nerve-injury-induced fiber sprouting. For this study, the well-described model of L5 spinal nerve transection was used because this model is known to be associated with primary afferent sprouting [6] and glial activation [18]. 


\section{Methods}

\section{Animals \& surgery}

All experiments were approved by the Animal Experiments Committee of the Maastricht University (DEC \# 2009-068), and were performed in accordance to the recommendations of the European Commission (European Communities Council Directive of 24 November 1986; 86/609/EEC). Sixty eight adult female Sprague-Dawley rats (Charles River, The Netherlands), weighing 205-240g were used for this experiment, of which three animals were excluded for further analysis. Two of the excluded animals displayed complete paralysis of the left hindpaw (possibly due to injury of the L4 spinal nerve) and one animal displayed paralysis of the right hindpaw following intrathecal catheter implantation. The number of animals used in each analysis is indicated in the figure legends. L5 spinal nerve transection was performed according to the method described by Chung et al. [21], with a slight modification. Animals were anesthetized with inhalation anaesthetics (induction: isoflurane 5\%; maintenance: 2\%, in air). Each animal was placed on a heated surgical platform $\left(37^{\circ} \mathrm{C}\right)$ and hairs of the lower back and left hindpaw were removed. After disinfection of the skin, a longitudinal incision was made at the lower lumbar and sacral levels exposing the paraspinal muscles. On the left side of the animal, muscles from the rostral part of the L5 vertebra up to the caudal part of L6 were separated. Connective tissues and remaining muscles were hold aside to expose the processus transversus of the L6 vertebra. Next, the L6 transverse process was removed, thereby exposing the $L 5$ spinal nerve. The $L 5$ spinal nerve was gently lifted and subsequently transected to ensure that all axons in the nerve were cut. A $5 \mathrm{~mm}$ piece of the $\mathrm{L} 5$ spinal nerve was resected to prevent spontaneous nerve regeneration. On completion of the operation, muscles were sutured using resorbable CatGut 4/0 thread and the skin was closed with nylon 3/0 sutures. The operation wound was disinfected and animals were returned to their home cages for recovery. Five days prior to L5 spinal nerve transection, a subgroup of rats had received an intrathecal (i.t.) catheter to administer daily bolus injections of either saline or propentofylline (PPF). For placement of the i.t. catheter, animals were anesthetized with inhalation anaesthetics (induction: isoflurane 5\%; maintenance: 2\%, in air). Each animal was placed on a heated surgical platform $\left(37^{\circ} \mathrm{C}\right)$ and the neck of the animal was shaven. The animal was placed in a stereotactic head-holder with the head flexed forward. After disinfection of the skin, a midline incision was made at the back of the occipital bone and the neck to expose the atlantooccipital membrane. The membrane was carefully opened by gently scraping the membrane with a curved 23-gauge needle, after which a polyethylene catheter (length of the catheter part to remain intrathecally: $8.5 \mathrm{~cm}$ ) was inserted and tunneled intrathecally up to the level of the L3-L5 spinal segments. The larger-diameter part of the catheter was tunneled subcutaneously to exit through the mid-dorsal aspect of the head. On completion of the operation, the skin was closed with nylon 3/0 sutures and the catheter was slowly flushed with $10 \mu \mathrm{L}$ of saline. The operation wound was disinfected and animals were returned to their home cages 
for recovery. Two days following implantation, $10 \mu \mathrm{L}$ of $2 \%$ lidocaine was injected intrathecally. Correct placement of the catheter was confirmed when lidocaine injection resulted in dragging or paralysis of both hindpaws. Following another 3 days of recovery, the i.t. catheter was used to daily administer bolus injections of saline or $10 \mu \mathrm{g}$ propentofylline per rat (PPF, Sigma). Treatment started 1 hour prior to L5 spinal nerve transection and was continued until day 5 post-injury. Animals were treated daily with saline or $10 \mu \mathrm{g}$ PPF in a volume of $20 \mu \mathrm{L}$, followed by an injection of $10 \mu \mathrm{L}$ saline to flush the catheter. Treatment with saline or PPF was blinded so that the experimenter recording the behavioral data was unaware of which rats were treated with saline or PPF.

\section{Behavioral testing}

Animals were tested on pre-operative day 1 and on post-operative days 3, 5, 7, 10 and 21. For testing static mechanical allodynia, the rats were placed in plastic, transparent cages with a wire mesh bottom. Before testing, acclimatization was allowed for approximately 15 min, until exploration and major grooming stopped. The dynamic plantar aesthesiometer (UgoBasile, Italy) was used to measure the paw withdrawal threshold (PWT). The test was performed by automated application of a metal filament to the mid-plantar surface of the hindpaw. The pressure increased $(0-50 \mathrm{~g})$ over time (10 s) until a withdrawal response of the hindpaw was elicited. Importantly, only withdrawal responses in which animals showed adversity to the stimulus (including paw licking, postural changes, and/or attacking of the stimulus) were considered. The test was repeated three times per animal per time point of investigation and results were averaged.

\section{Non-nociceptive stimulation of L5 SNT animals}

Five days following injury, a subgroup of L5 SNT animals received non-nociceptive stimulation as described earlier [22]. In brief, animals were gently held by the investigator and the rat's plantar hindpaw was rubbed using the flat portion of the investigator's thumb. Strokes were made towards the distal footpad over a period of $2 \mathrm{~s}$. This procedure was done for both hindpaws and for a total duration of 10 minutes. Animals were sacrificed 2 hours after the 10-min-stimulation period. A subgroup of L5 SNT animals in which the hindpaws were not stimulated served as controls.

\section{Tissue processing}

Rats were anesthetized with an overdose of pentobarbital (150 mg/kg body weight) via intraperitoneal injection and subsequently transcardially perfused with $0.1 \mathrm{M}$ phosphate-buffered saline (PBS) followed by a 4\% paraformaldehyde/15\% picric acid solution (PF-P). Lumbar spinal cord sections were identified, removed and post-fixed in PF-P overnight at $4^{\circ} \mathrm{C}$. The 
lumbar spinal cords were cryoprotected by overnight incubation in 10\% sucrose solution (in $0.1 \mathrm{M}$ phosphate buffer; $\mathrm{pH} 7.6$; at $4^{\circ} \mathrm{C}$ ), followed by at least $72 \mathrm{~h}$ incubation in $30 \%$ sucrose solution (in $0.1 \mathrm{M}$ phosphate buffer; $\mathrm{pH} 7.6$; at $4^{\circ} \mathrm{C}$ ). The spinal cord segments were frozen using dry ice powder and stored at $-80^{\circ} \mathrm{C}$ until further processing. For cryosectioning, spinal cords were mounted in Tissue-Tek Optimal Cutting Temperature solution (O.C.T., Sakura FineTek). Thirty $\mu \mathrm{m}$ serial sections were mounted on gelatin-coated glass slides and stored at $-20^{\circ} \mathrm{C}$ until immunohistochemical processing.

\section{Immunohistochemistry}

Glass slides were thawed for about 2 hours at room temperature followed by three washing steps of 10 minutes each with PBS. Next, spinal sections were incubated with the following primary antibodies: rabbit anti-PKCY (1:1000, Santa Cruz Biotechnology), mouse antiGAP-43 (1:16000, Chemicon) as a sprouting marker, rabbit anti-CGRP (1:1000, Biotrend) for peptidergic nociceptive fibers, rabbit anti-VGLUT-1 (1:1000, Synaptic Systems) for A $\beta$ fibers, rabbit anti-c-Fos (1:2000, Santa Cruz Biotechnology) as a marker for early neuronal activation. Biotinylated IB4 (1:1000, Sigma) was used to mark nonpeptidergic nociceptive fibers. All primary antibodies were diluted in PBS-T (PBS with 0.3\% Triton X-100) and incubated overnight at room temperature. The following day, sections were rinsed three times for 10 minutes each with PBS. Next, sections were incubated with the following secondary antibodies: Alexa594-conjugated donkey anti-rabbit (for PKCY, CGRP and VGLUT-1), Alexa488conjugated donkey anti-mouse (for GAP-43 and c-Fos) and 594-conjugated streptavidin (for IB4). All secondary antibodies were diluted in PBS-T (1:100, Invitrogen) and incubated for 2 hours at room temperature. Spinal sections were washed again three times for 10 minutes each (PBS) and embedded in 80\% glycerol/PBS. For double-labelling experiments, spinal sections were incubated with a mixture of the two respective primary antibodies in PBS-T overnight at room temperature. The secondary antibodies were also mixed and sections were incubated with this mixture for 2 hours at room temperature. For GFAP immunohistochemistry, glass slides were thawed for about 2 hours at room temperature followed by three washing steps of 10 minutes each with the following solutions: TBS-T (Tris-BufferedSaline containing 1\% Triton X-100), TBS and TBS-T, respectively. Next, spinal sections were incubated with rabbit anti-GFAP (1:1000, DAKO) in TBS-T overnight at room temperature. The following day, sections were rinsed three times for 10 minutes each with TBS-T, TBS and TBST. Next, sections were incubated with the Alexa488-conjugated donkey anti-rabbit (1:100 in TBS-T) and incubated at room temperature for 2 hours. Spinal sections were washed again three times for 10 minutes each in TBS and embedded in 80\% glycerol/TBS. For iba-1 immunohistochemistry, glass slides were thawed for about 2 hours at room temperature followed by three washing steps of 10 minutes each with the TBS. Next, spinal sections were incubated with blocking serum (TBS-T containing 5\% normal donkey serum (NDS)) for 1 hour at $4^{\circ} \mathrm{C}$. Next, sections were incubated with rabbit anti-iba-1 (WAKO, 1:1000 in TBS-T 
containing $1 \%$ NDS) overnight at $4^{\circ} \mathrm{C}$. The following day, sections were rinsed three times for 10 minutes each with TBS. Next, sections were incubated with the Alexa488-conjugated donkey anti-rabbit (1:100 in TBS-T) and incubated for 1 hour at $4^{\circ} \mathrm{C}$. Spinal sections were washed again three times for 10 minutes each (TBS) and embedded in 80\% glycerol/TBS. Double immunohistochemistry for PKCY and c-Fos was performed by sequential immunostaining as described earlier [4]. In brief, sections were first incubated with rabbit anti-c-Fos (1:1000, Santa Cruz Biotechnology) followed by incubation with the secondary antibody biotin-conjugated donkey anti-rabbit. c-Fos immunoreactivity was visualized using nickelDAB. Next, sections were incubated with rabbit anti-PKCY (1:1000, Santa Cruz Biotechnology) followed by incubation with the secondary antibody biotin-conjugated donkey antirabbit. PKCY immunoreactivity was visualized using DAB only.

\section{Image analysis}

GAP-43 immunostained sections were examined under a fluorescent microscope (Olympus). Photomicrographs of the ipsilateral and contralateral dorsal horn were taken of immunostained sections at a 10x objective (and a 12.5× condenser) using a grayscale F-view camera. Analysis was performed using the software program CellP (Olympus). For measuring GAP-43 immunoreactivity, regions of interest (ROI) were drawn on each dorsal horn. Based on the photomicrographs of PKC $Y$-immunoreactive sections, two different ROls were delineated: 1) a small band in the dorsal horn displaying strong PKCY-immunoreactivity was used to determine the PKC $\gamma$ layer itself. This layer is further indicated as $\|_{\mathrm{iv} \text {, }}$ as PKC -positive interneurons lie in a dense band corresponding to the most ventral part of the inner lamina $\|$; 2) The remaining gray matter of the dorsal horn above the PKC -positive layer (or $\|_{i v}$ ) was delineated and assigned as layers $1-\|_{\mathrm{id}}$, corresponding to the area covering lamina I, outer lamina II and the most dorsal part of inner lamina II. These ROls were then saved and copied to the photomicrographs of GAP-43 immunostained sections. This allowed us to measure GAP-43 immunoreactivity in the layers $I-\|_{\text {id }}$ and $\|_{i v}$. The mean gray value within each $R O I$ was measured to quantify the GAP-43 immunoreactivity (resulting in a gray value between 0 and 255). Per animal, an average of 12 spinal sections was analyzed for GAP-43 immunoreactivity. Results are expressed as a percent difference in gray value between ipsi- and contralateral dorsal horn. To investigate the double-labeling of GAP-43 (green) and the different primary afferents markers (red), individual photomicrographs were taken using a fluorescent microscope (Olympus) and two photomicrographs were merged together using CellP. To quantify the GFAP and iba-1 immunoreactivity signal, photomicrographs of the ipsilateral and contralateral dorsal horn were taken of the immunostained sections at a $4 \times$ objective (and a $12.5 \times$ condenser) using a grayscale F-view camera. Analysis was performed using CellP. ROls were drawn of the ipsilateral and contralateral dorsal horn and the mean gray value within each $\mathrm{ROI}$ was measured. Results are expressed as a percent difference in gray value between the ipsilateral and contralateral dorsal horn. 


\section{Stereology-based cell counting}

Stereology-based cell counting was performed with a stereology workstation consisting of a modified light microscope (Olympus BX50) and stereology software (Stereolnvestigator, MBF Bioscience). Total numbers of c-Fos positive cells were evaluated with the Optical Fractionator [23]. Consecutive sections were used to count c-Fos positive cells in both lamina I-II and $\|_{\mathrm{iv}}$ of the $L 4$ and $L 5$ spinal dorsal horn. First, ROls were drawn at a 10x magnification based on the sections immunostained for PKCy. These ROls were saved and transferred to the consecutive section immunostained for c-Fos. Using the Optical Fractionator, c-Fos positive cells were counted at a 20x magnification in a counting frame of $22500 \mu^{2}$ and with an optical dissector height of $16 \mu \mathrm{m}$. Fixed distances (guard zones) of $2 \mu \mathrm{m}$ were set to eliminate counting the same cell twice. All neurons whose nucleus top came into focus within unbiased virtual counting spaces distributed in a systematic-random fashion throughout the delineated regions were counted [24]. Then, total neuron numbers were calculated from the density of counted neurons and the corresponding corrected counting volume.

\section{Confocal microscopy}

Confocal images of GAP-43 and CGRP double immunohistochemistry were analyzed with a Confocal Spinning Disk (SI-SD) system (MBF Bioscience; Williston, VT), consisting of a BX51 microscope (Olympus), a customized spinning disk unit (DSU; Olympus) and controlling software (Stereolnvestigator, MBF Bioscience). A digital RGB image stack showing the same microscopic field in 47 consecutive focal planes with a distance of $0.5 \mu \mathrm{m}$ between the focal planes were generated with the $100 \times$ objective. From these image stacks, three-dimensional reconstructions (maximum intensity projections) were produced with Imaris 4.0 software.

\section{Statistics}

All data were analyzed with the statistical program SPSS 15.0. Statistical comparisons between baseline and post-injury paw withdrawal thresholds were performed with an unpaired Student's t-test. GAP-43 immunoreactivity in injured animals was compared with sham-operated animals by using an one-way ANOVA with a Dunnett post-hoc test. Gray values of iba- 1 and GFAP immunoreactivity and the total number of c-Fos positive cells between stimulated and non-stimulated animals were compared using an unpaired Student's t-test. All data are expressed as mean \pm sem. A p-value of 0.05 was regarded as the level of statistical significance. 


\section{Results}

\section{Transection of the L5 spinal nerve results in static mechanical allodynia}

Transection of the L5 spinal nerve resulted in a marked decrease of the ipsilateral PWT from DPO 3 onwards and lasting until at least DPO 21 (Fig 1). From DPO 3 onwards until DPO 21, the ipsilateral PWT was significantly decreased compared to the contralateral PWT (ranging from -58.4 to -47.6\%). Sham-operated animals displayed no significant decreases in ipsi/contra ratios $(-8.9 \pm 6.1 \%)$ compared to baseline ratios $(-2.2 \pm 10.5 \%)$.

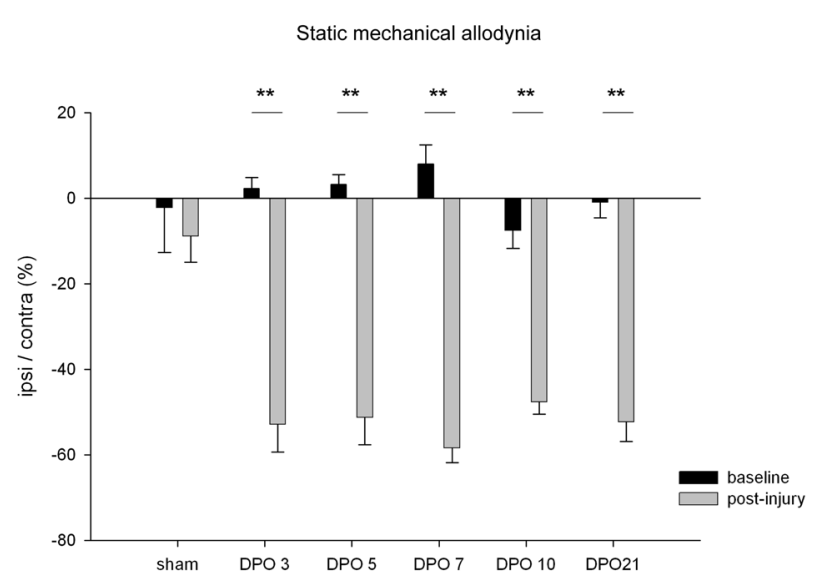

Figure 1. Static mechanical allodynia following L5 SNT. PWT of animals subjected to L5 SNT were measured before the injury (baseline) and up to DPO 21. Results are expressed as percent decrease of the ipsilateral PWT compared to the contralateral PWT. Post-injury ipsi/contra ratios of PWTs were significantly decreased compared to baseline ipsi/contra ratios from DPO 3 up to DPO 21. Sham-operated animals displayed no significant differences between baseline and postoperative ipsi/contra ratios. $\mathrm{N}=4$ for sham, $\mathrm{n}=5$ for DPO $21, \mathrm{n}=6$ for DPO 3, 5, and $7, \mathrm{n}=10$ for DPO 10. ${ }^{* *} \mathrm{p}<0.01$. PWT, paw withdrawal threshold; SNT, spinal nerve transection; DPO, day post-operative.

\section{Non-nociceptive stimulation of the hindpaw increases cellular activation}

c-Fos positive cells were counted in both $1-\|_{\text {id }}$ and $\|_{\text {iv }}$ of L5 SNT animals with and without nonnociceptive hindpaw stimulation (Fig 2). Animals without stimulation displayed relatively low numbers of c-Fos positive cells in the spinal dorsal horn (Fig 2A,B,F). No differences were noted between the total number of cells in the ipsilateral and contralateral $\|_{\text {iv }}$ or $1-\|_{\text {id }}$ in nonstimulated L5 SNT animals (Fig 2A,B,F). Non-nociceptive stimulation of the hindpaws of L5 SNT animals significantly increased the total number of c-Fos positive cells in both the $\|_{\text {iv }}$ 
layer (326.1 \pm 43.9 versus $152.9 \pm 29.4$ in non-stimulated L5 SNT animals) and the I- I $_{\text {id }}$ layer (754.0 \pm 69.9 versus $365.4 \pm 39.9$ in non-stimulated L5 SNT animals) of the ipsilateral spinal dorsal horn (Fig 2). c-Fos immunoreactive profiles within the PKCY-positive spinal layer were largely restricted to the PKCY-positive interneurons (Fig 2E).

\section{Spinal nerve transection induces sprouting of CGRP-positive fibers at the L4 and L5 dorsal horn}

Representative fluorescent images of GAP-43 expression in $\|_{\mathrm{iv}}$ layers and $\mathrm{I}-\mathrm{I}_{\mathrm{id}}$ layers are shown in Figure 3. Ipsilateral and contralateral GAP-43 expression in sham-operated animals were comparable (Fig 3A,B). At the L4 lumbar level, GAP-43 expression was highly upregulated in the ipsilateral I- $\|_{\text {id }}$ and $\|_{\text {iv }}$ layer compared to the contralateral I- $\|_{\text {id }}$ and $\|_{\text {iv }}$ layer (Fig 3C,D). At the

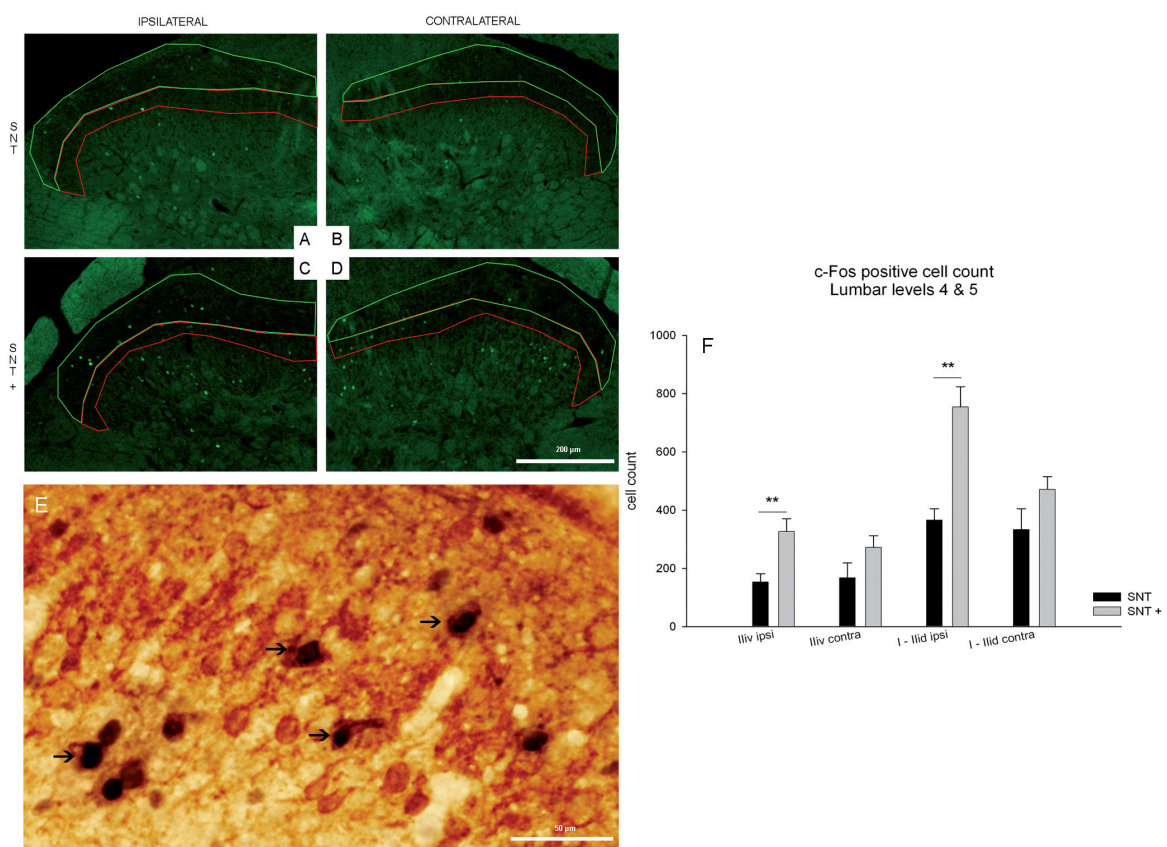

Figure 2. $C$-Fos positive cell count in both $\|_{\mathrm{iv}}$ and $I-I_{\mathrm{id}}$ layers at the $L 4$ and $L 5$ spinal level. A subgroup of $L 5$ SNT animals were subjected to touch stimulation of both hindpaws to induce the expression of the immediate early gene c-Fos in the spinal cord (SNT +). (A,C,F) At the L4 and L5 spinal levels, animals receiving touch stimulation (SNT +) showed significant increases in the total number of c-Fos positive cells in both ipsilateral $\|_{\mathrm{iv}}$-and $\mathrm{I}-\mathrm{I}_{\mathrm{id}}$ layers compared to the ipsilateral $\|_{\mathrm{iv}}$-and $I-I_{\text {id }}$ layers of non-stimulated animals (SNT). (B,D,F) No significant differences in the total number of c-Fos positive cells were observed between the contralateral $\|_{\mathrm{iv}}$-and $I-\|_{\mathrm{id}}$ layers of stimulated and non-stimulated animals. (E) Within the $\|_{\mathrm{i}}$ layer, c-Fos was found to be highly co-localized with PKC -positive interneurons (arrows). $n=6$ for SNT and $n=6$ for SNT+. ** $p<0.01$. PKC $\gamma$, protein kinase C gamma; SNT, spinal nerve transection. 
L5 lumbar level, GAP-43 expression was significantly increased in the ipsilateral $\|_{\text {iv }}$ and I- $\|_{\text {id }}$ layers compared to the contralateral $\|_{\text {iv }}$ and $I-\|_{\text {id }}$ layers (Fig 3E,F). At DPO 21, ipsilateral GAP-43 expression returned towards levels of the contralateral dorsal horn (Fig 3G,H).

GAP-43 expression at lumbar levels L3 to L5 of sham-operated and L5 SNT animals were quantified in both the $\|_{\text {iv }}$ layer as well as I- $\|_{\text {id }}$ layers (Fig 4). At the L3 lumbar level, post-injury GAP-43 expression was never increased compared to sham-operated animals (Fig 4A,B). At the L4 lumbar level, GAP-43 expression was strongly increased, although this level never reached statistical significance (Fig 4C) (II layer, DPO 7: $145.9 \pm 17.0 \%$ versus 107.1 $\pm 12.5 \%$; $p=0.07)\left(I-\|_{\text {id }}\right.$ layers, DPO 5: $138.2 \pm 7.9 \%$ versus $\left.107.3 \pm 15.0 \% ; p=0.09\right)$. At the $L 5 \|_{\text {iv }}$ layer (Fig 4E), GAP-43 expression was significantly upregulated at DPO $5(123.8 \pm 5.7 \% ; \mathrm{p}<0.05)$, DPO 7 (122.9 $\pm 6.3 \% ; p<0.05)$ and DPO 10 (120.5 $\pm 4.6 \% ; p<0.05)$ compared to sham-operated animals (96.8 $\pm 9.3 \%)$. At the $\mathrm{L} 5 \mathrm{I}-\mathrm{II}_{\text {id }}$ layers (Fig 4F), GAP-43 expression was significantly upregulated at DPO $5(122.6 \pm 5.1 \% ; p<0.05)$ and DPO $7(120.8 \pm 4.5 \% ; p<0.05)$ and was still increased at DPO $10(116.2 \pm 7.3 \% ; p=0.08)$ compared to the GAP-43 expression in shamoperated animals $(93.6 \pm 11.3 \%)$. GAP-43 expression in $\|_{\text {iv }}$ layer $(111.8 \pm 3.8 \%)$ as well as $1-\|_{\text {id }}$ layers (108.2 $\pm 2.8 \%$ ) of the L5 lumbar level returned to expression levels of sham-operated animals at DPO 21.

Double immunohistochemistry revealed double-labeling (yellow) of GAP-43 with CGRP both within and above the $\|_{\text {iv }}$ layer at lumbar levels $L 4$ and L5 (Fig 5D). No co-localization was found between GAP-43 and PKCY (Fig 5A), VGLUT-1 (Fig 5B) or IB4 (Fig 5C). Separate fluorescent images of GAP-43 (Fig 5E) and CGRP (Fig 5F) of the same region showed fibers which clearly stained for both markers. Confocal analysis of fiber-like structures revealed unambiguous evidence for co-localization of GAP-43 and CGRP (Fig 5G).

\section{PPF is effective in reducing both microglial activation in the spinal dorsal horn and static mechanical allodynia but ineffective in reducing spout- ing responses following spinal nerve transection}

Daily intrathecal bolus injections of PPF at 5 consecutive days following L5 SNT effectively attenuated microglial activation (Fig 6). Saline-treated L5 SNT animals displayed robust upregulation of iba-1, a microglial marker, in the ipsilateral dorsal horn (Fig 6B) compared to the contralateral dorsal horn (Fig 6A). Daily intrathecal bolus injections of PPF for a period of 5 days significantly reduced iba-1 expression in the dorsal horn of L5 SNT animals (Fig 6D). The ipsi/ contra ratio of iba-1 expression was significantly reduced in PPF-treated animals compared to saline-treated animals (123.4 $\pm 2.3 \%$ versus $131.6 \pm 1.1 \% ; p<0.05)$. No significant change in GFAP, marker for astroglial activation, could be observed at this time point (5 days post-L5SNT) in the ipsilateral dorsal horn (Fig 6G) compared to the contralateral dorsal horn (Fig 6F). Consequently, PPF treatment did not affect GFAP expression in the dorsal horn (Fig 6I). Quantification of GFAP expression showed no significant change of the ipsi/contra ratio in PPF-treated animals compared to saline-treated animals (105.9 $\pm 3.9 \%$ versus $108.3 \pm 1.7 \%$, Fig $6 \mathrm{~J}$ ). 


\section{IPSILATERAL}
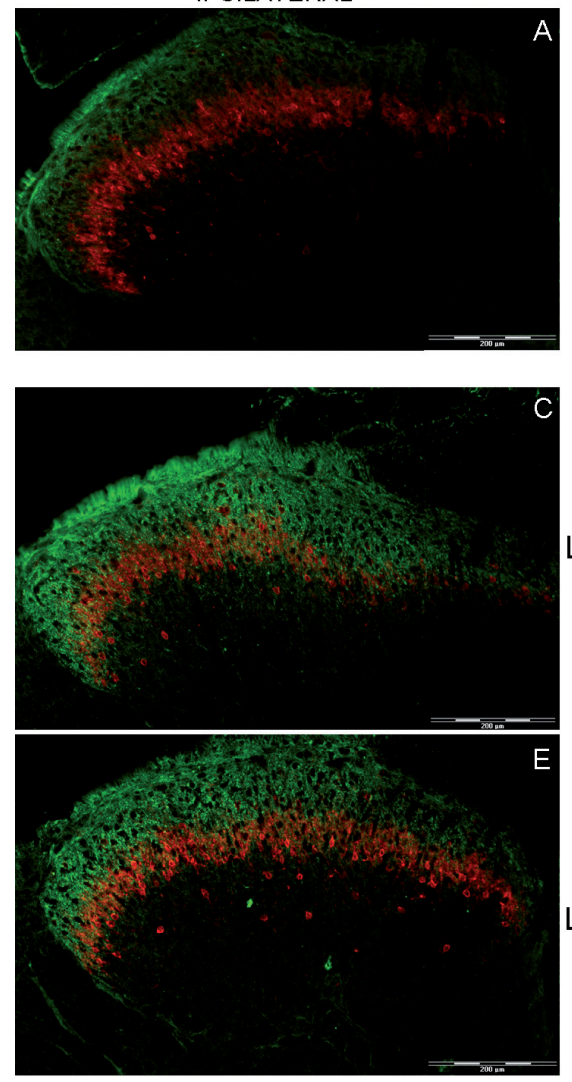

E

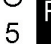

L5

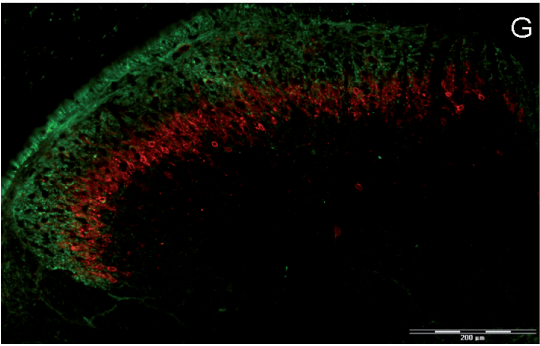

$\mathrm{G}$

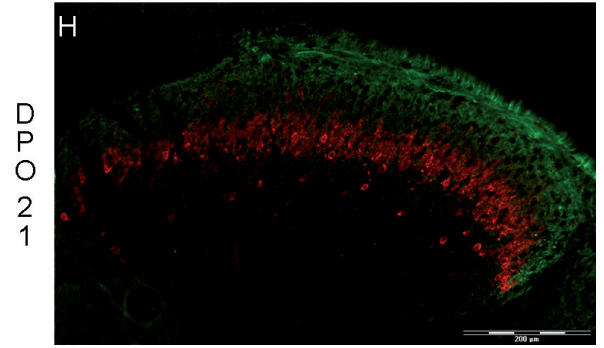

Figure 3. Differential GAP-43 expression in the dorsal horn of sham-operated and nerve-injured animals. $(A, B)$ The ipsilateral and contralateral dorsal horn of sham-operated animals displayed comparable levels of GAP-43 immunoreactivity (green). (C,D) At DPO 5, GAP-43 immunoreactivity (green) was increased in both the $\|_{\text {iv }}$ layer (in red) and the I-II id $_{\text {layers of }}$ the ipsilateral dorsal horn compared to the contralateral side at the L4 spinal level. (E,F) At DPO 5, GAP-43 immunoreactivity (green) was significantly upregulated in both the $\mathrm{II}_{\mathrm{i} \text { I }}$ layer (in red) as in the I-II layers of the ipsilateral dorsal horn compared to the contralateral side at the L5 spinal level. (G,H) At DPO 21, GAP-43 immunoreactivity of the ipsilateral dorsal horn was reduced to levels similar to the contralateral side. GAP-43, growth associated protein-43; SNT, spinal nerve transection; DPO, day post-operative. 


\section{Lumbar level L3}
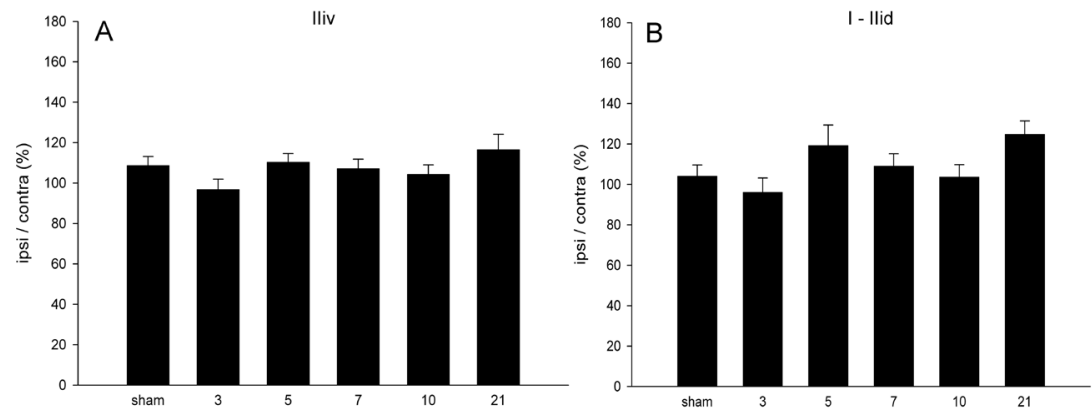

Lumbar level L4
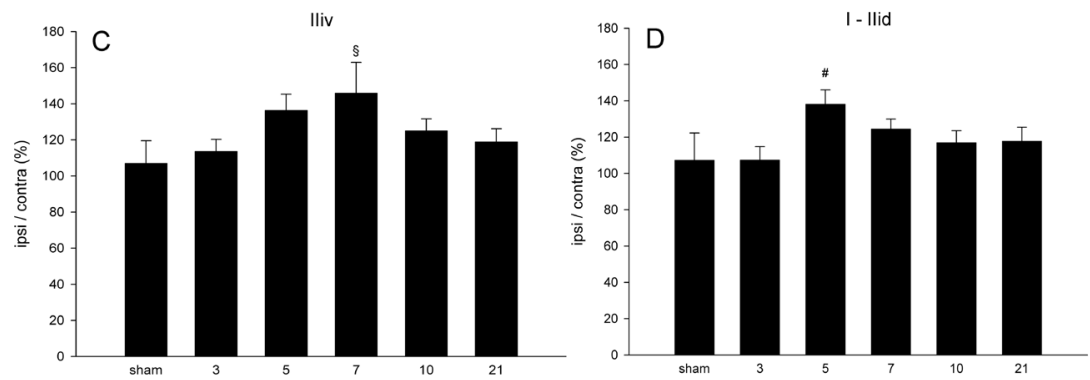

Lumbar level L5
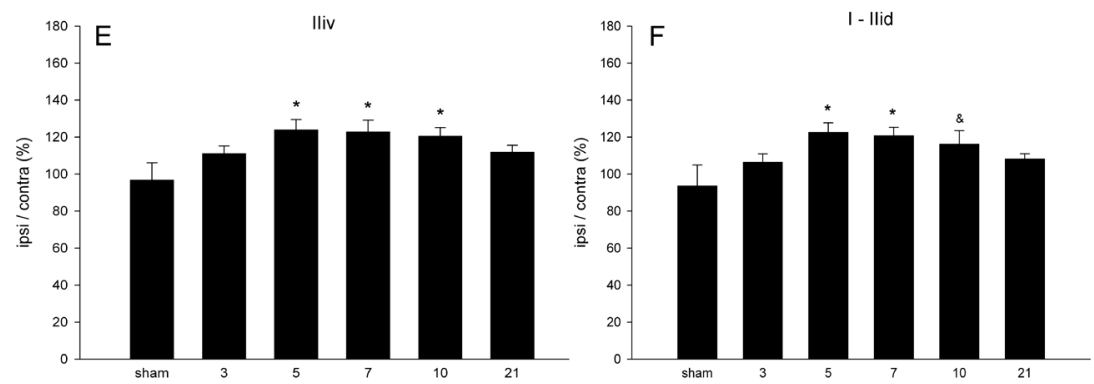

Figure 4. Temporal sprouting responses at different lumbar spinal levels in both $\|_{i v}$-and $I-\|_{\text {id }}$ layers of the dorsal horn. GAP43 expression was quantified by measuring the ratio of immunoreactivity between the ipsilateral and contralateral $\|_{\text {iv }}$-and $1-I_{\text {id }}$ layers of the dorsal horn at the lumbar levels L3, L4 and L5 following L5 SNT. (A,B) At the L3 spinal level, no significant increase in GAP-43 expression was observed at the $\|_{\text {iv }}$ layer or at the I- II layers of L5 SNT animals compared to shamoperated animals. (C,D) At the L4 spinal level, a strong but statistically non-significant upregulation of GAP-43 expression was observed at the $\|_{i v}$ layer $(p=0.07)$ and $I-I_{i d}$ layers $(p=0.09)$ of L5 SNT animals compared to sham-operated animals. $(E, F)$ At the L5 spinal level, L5 SNT resulted in a statistically significant upregulation of GAP-43 expression in the II layer at DPO 5, 7 and 10. GAP-43 expression was also significantly increased in the superficial layers at DPO 5 and $7 . n=4$ for sham, $\mathrm{n}=5$ for DPO 21, $\mathrm{n}=6$ for DPO 3, 5, and $7, \mathrm{n}=7$ for DPO 10. ${ }^{*} \mathrm{p}<0.05, \S p=0.07, \# p=0.09, \& p=0.08$. GAP-43, growth associated protein-43; SNT, spinal nerve transection; DPO, day post-operative. 

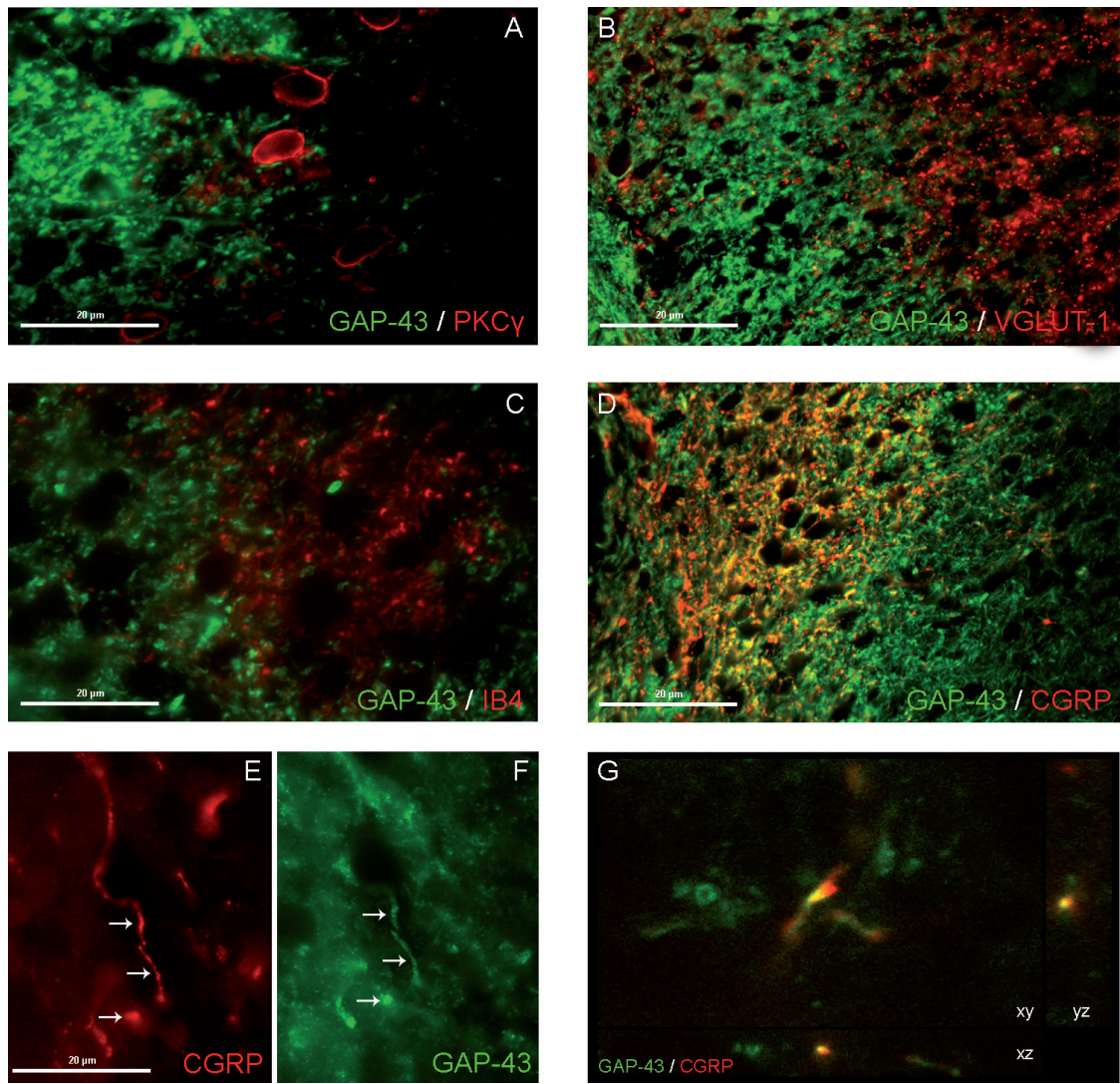

Figure 5. Double immunohistochemistry for the identification of sprouting (GAP-43 immunoreactive) fibers. Combined immunohistochemical stainings for GAP-43 (green) with PKCY (A) and the primary afferent markers VGLUT-1 (B), IB4 (C) and CGRP (D) (all in red) at DPO 5 following L5 SNT. GAP-43 expression was found to be exclusively co-localized (yellow) with the primary afferent marker CGRP (D). High magnification of GAP-43 (green) and CGRP (red) in the $\|_{\text {iv }}$-and I- $\|_{\text {id }}$ layers showed overlap in stainings for fiber-like structures (indicated by arrows). (G) Confocal analysis revealed an unambiguous co-localization of both GAP-43 and CGRP. GAP-43, growth associated protein-43; SNT, spinal nerve transection; PKC $\gamma$, protein kinase C gamma; VGLUT-1, vesicular glutamate transporter 1; IB4, isolectin B4; CGRP, calcitonin gene-related protein; $\mathrm{DPO}$, day post-operative. 

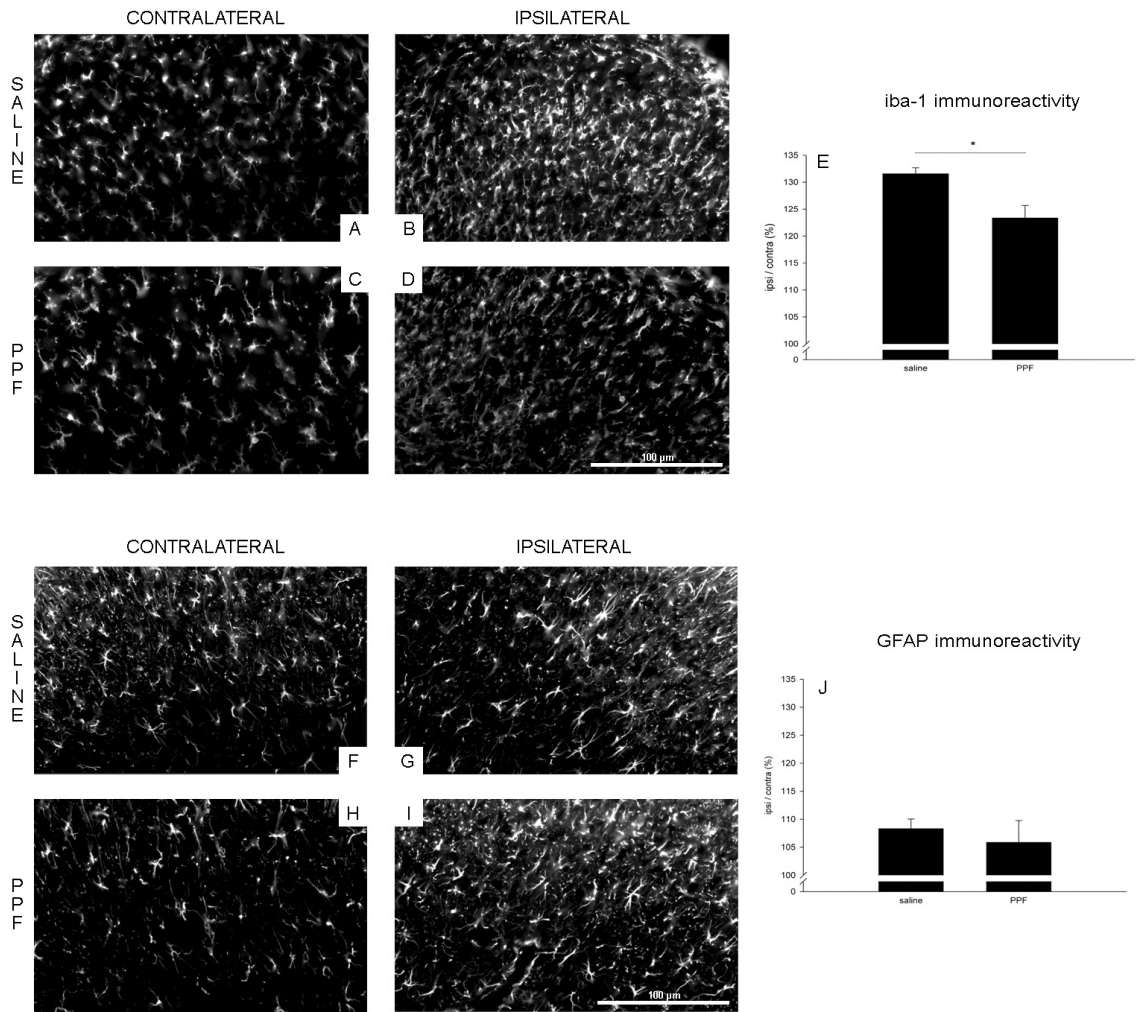

Figure 6. Iba-1 and GFAP immunoreactivity in the dorsal horn of L5 SNT animals following i.t. administration of saline or PPF. PPF treatment resulted in a clear reduction of iba-1 immunoreactivity in the ipsilateral dorsal horn (D) compared to the ipsilateral dorsal horn of saline-treated L5 SNT animals (B). (E) Quantification of iba-1 immunoreactivity in L5 SNT animals revealed a significant reduction following PPF treatment compared to saline-treatment. As GFAP immunoreactivity was not significantly increased in the ipsilateral dorsal horn $(G)$ versus the contralateral dorsal horn of nerve-injured animals (F), PPF treatment also did not affect GFAP immunoreactivity (H-I). (J) Quantification of GFAP immunoreactivity in L5 SNT animals revealed no significant changes in GFAP expression between PPF treated and saline-treated animals. (E) $n=4$ for saline and $n=5$ for PPF. $(J) n=5$ for saline and $n=5$ for PPF. ${ }^{*} p<0.05$. SNT, spinal nerve transection; PPF, propentofylline; iba-1, ionized calcium binding adaptor molecule 1; GFAP, glial fibrillary acidic protein. 
The attenuation of microglial activation following PPF treatment in L5 SNT animals was paralleled by an attenuation of static mechanical allodynia (Fig 7). At DPO 5, L5 SNT animals treated with PPF displayed a less pronounced decrease in ipsi/contra ratios of PWT in comparison to saline-treated L5 SNT animals (-26.6 \pm 5.7 versus $-57.3 \pm 3.0 ; p<0.01$; Fig 7C).

Despite the significant effect of PPF treatment on microglial activation and static mechanical allodynia, no effect of PPF was noted on the GAP-43 expression following L5 SNT. At DPO 5 , GAP-43 expression in the $\|_{\mathrm{iv}}$ layer as well as the $1-\|_{\text {id }}$ layer was at the same level in PPF and saline treated animals, both significantly increased compared to sham-operated animals (Fig 7A,B).

\section{Discussion}

In this study, we report increases in the total number of c-Fos positive cells within and above the $\|_{\text {iv }}$ layer of the lumbar spinal cord following non-nociceptive hindpaw stimulation of $L 5$ spinal nerve transected animals. Concurrently, we observed a time-specific upregulation of GAP-43 expression within these spinal layers. GAP-43 immunoreactive fibers were found to be highly co-localized with the primary afferent marker CGRP. Although intrathecal PPF treatment significantly attenuated microglial iba-1 expression in the ipsilateral dorsal horn and static mechanical allodynia following L5 spinal nerve transection, PPF treatment did not reduce the injury-induced GAP-43 expression.

Mechanisms underlying mechanical allodynia following peripheral nerve injury are still under investigation. To date, several concepts have been proposed to contribute to mechanical allodynia (Fig 8). A first concept involves direct non-nociceptive input towards NS projection neurons (Fig $8 B$ ). It was suggested that non-nociceptive primary afferents (i.e. $A \beta$ fibers) sprout from their normal termination area (i.e. $\|_{\text {iv }}$ and deeper layers) to more superficial layers (i.e. $1-\|_{\text {id }}$ layers) of the spinal dorsal horn, which contain NS projection neurons $[7,8,9,25,26,27]$. The observations of such $A \beta$ sprouting responses are, however, largely based on fiber tracing bias $[28,29,30]$. More recent studies using specific labelling of primary A $\beta$ afferents like single-fiber analysis and intra-axonal labelling provide little, if any, evidence for $A \beta$ sprouting beyond inner lamina II [31,32].

A second concept involves indirect activation of NS projection neurons upon A $\beta$ input (Fig 8 C-E). This indirect pathway is mediated via a local circuit comprising PKCY-positive interneurons which are connected, presumably polysynaptically, to lamina I NS projection neurons. PKC $\gamma$-positive interneurons selectively receive non-nociceptive A $\beta$ fiber input [2], and under normal circumstances this input is effectively not gated to NS projection neurons and as such not perceived as painful [4] (Fig 8A). In neuropathic conditions, activation of this circuit may occur as a result of (1) sensitization or (2) disinhibition of the circuit.

Sensitization of the circuit may be induced by 2 different mechanisms. First, enhanced activity resulting from ectopic impulses from injured $A \beta$ fibers [33] and to a lesser extent, if any, from injured $C$ fibers $[34,35,36]$ may be involved in sensitization (Fig $8 C$ ). The ectopic 


\section{Lumbar level L5}
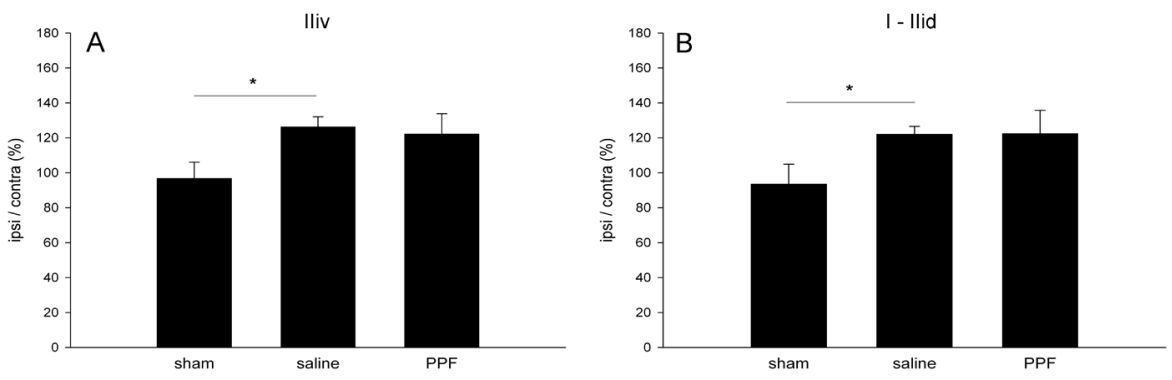

\section{Static mechanical allodynia}

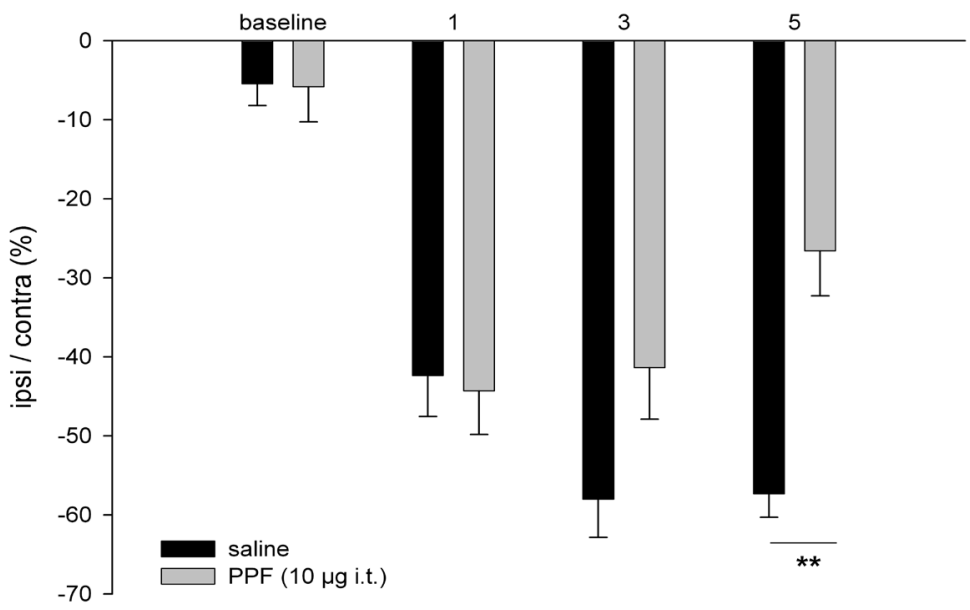

Figure 7. Sprouting responses at the L5 spinal level and static mechanical allodynia in L5 SNT animals treated with saline or PPF. GAP-43 expression was quantified at DPO 5 by measuring the ratio of immunoreactivity between the ipsilateral and contralateral $\|_{\mathrm{iv}}$ layers as well as in the $\mathrm{I}-\mathrm{I}_{\mathrm{id}}$ layers of the dorsal horn at the lumbar level L5 following L5 SNT. (A,B) A significant increase in GAP-43 expression in both $\|_{i \mathrm{i}}$-and $I-\|_{\text {id }}$ layers was noted between sham-operated and saline-treated L5 SNT animals. Intrathecal administration of PPF did not affect GAP-43 expression. Although GAP-43 expression was not reduced following PPF treatment, static mechanical allodynia was significantly reduced at DPO 5 in PPF-treated animals compared to saline-treated animals (C). (A,B) $n=4$ for sham, $n=5$ for saline and $n=5$ for PPF. (C) $n=10$ for saline and $n=12$ for PPF. ${ }^{*} p<0.05 .{ }^{* *} p<0.01$. PPF, propentofylline; SNT, spinal nerve transection; GAP-43, growth associated protein 43; DPO, day post-operative. 


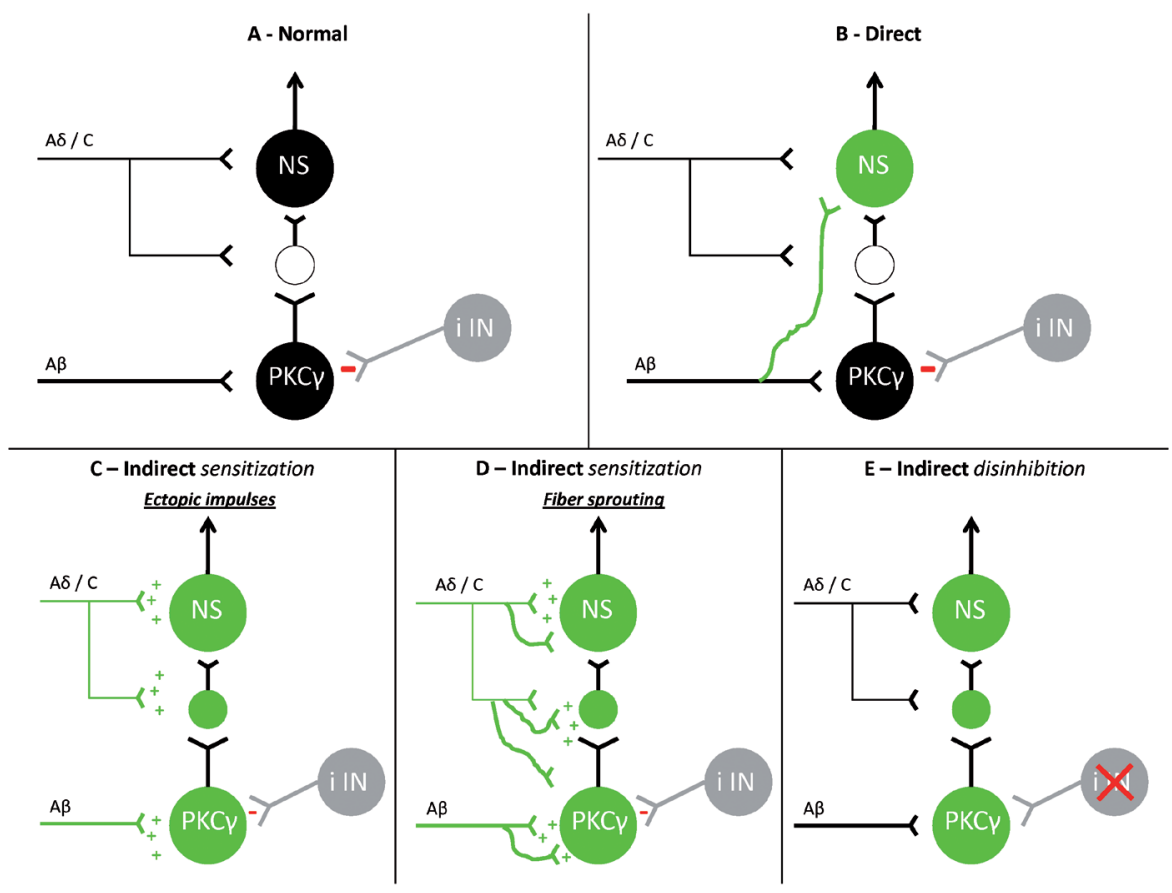

Figure 8. Concepts of nerve-injury induced mechanical allodynia. In the dorsal spinal horn, a local, presumably polysynaptic, circuit exists between A $\beta$ fiber-innervated PKC $\gamma$-positive interneurons and NS projection neurons. (A) Under normal conditions, A $\beta$ primary afferent input does not activate NS projection neurons as the circuit is under glycinergic/GABAergic inhibition (i IN). (B) NS projection neurons may be activated via direct input of A afferents, which sprout from their normal termination area into more superficial layers. (C-E) NS projection neurons may also be indirectly activated by sensitization or disinhibition of the circuit. Sensitization of the circuit may occur as a result of enhanced excitatory input, which is mediated by ectopic impulses generated by injured or intact primary afferents (C) or sprouting primary afferent fibers (D). This sensitization ultimately overrules the glycinergic/GABAergic inhibition. (E) NS projection may also be activated upon $A \beta$ input due to disinhibition, in which the glycinergic/GABAergic inhibition becomes dysfunctional and allows transmission of A $\beta$ input via PKC -positive interneurons towards NS projection neurons. Green coloration represents sensitization/activation. NS, nociception-specific; PKC , protein kinase C gamma; ilN, inhibitory interneuron. 
impulses are generated at the neuroma [37] or in the cell body of injured DRG neurons [38]. Moreover, the remaining uninjured C fibers also develop ectopic discharges [39] and were found to be crucial in the initiation and maintenance of injury-induced mechanical allodynia [35]. Second, circuit sensitization may be mediated by enhanced input due to sprouting of primary afferents (Fig 8D). 3D reconstructions of the spinal cord have previously revealed sprouting responses of intact $L 4$ nociceptive fibers into the $L 5$ dorsal horn following ligation of the L5 spinal nerve [6].

Disinhibition of the circuit has been reported to result in mechanical allodynia [4] (Fig 8E). Under physiological condition, glycine inhibits PKCY-positive interneurons, thereby preventing transmission of A $\mathrm{\beta}$ fiber input to NS projection neurons. Pharmacological interference with glycinergic inhibition resulted specifically in dynamic mechanical allodynia [4]. In contrast, static mechanical allodynia was shown to be related to GABAergic inhibition dysfunction [5]. The concept of disinhibition may also account for the static mechanical allodynia observed in the present study, possibly mediated via GABAergic disinhibition [40].

In this study, increased c-Fos immunoreactivity within and above the $\|_{\text {iv }}$ layer may imply the activation of the normally inactive circuit between A $\beta$ fibers and NS projection neurons as a result of sensitization mediated by aberrant sprouting of CGRP-positive fibers. The resultant sensitization of neurons located in the $\|_{\text {iv }}$ layer, due to the enhanced input of CGRP-positive nociceptive afferents, may increase the net cellular activity in this spinal layer following $A \beta$ input, thereby overruling inhibition of the circuit and ultimately inducing activation of NS projection neurons located in lamina I. We clearly showed that most c-Fos immunoreactive profiles within the $\|_{i v}$ layers were PKC $Y$-positive interneurons. The characterization of c-Fos immunoreactive profiles in the $1-\|_{\text {id }}$ layers remains unclear, as it was shown that NS projection neurons of the circuit are NK1-negative [5]. A $\beta$ fiber stimulation via touch stimulation of the hindpaws significantly increased ipsilateral c-Fos immunoreactive cell numbers in the $1-\|_{\text {id }}$ layers of the dorsal horn. These results suggest that injury-induced sprouting of CGRP-positive primary afferents sensitizes the circuit and gates A $\beta$ fiber input to NS projection neurons. Earlier, electrophysiological recording revealed such increases of polysynaptic $A \beta$ input to superficial neurons following peripheral nerve injury [41] and in the setting of pharmacological blockade with GABAergic inhibition [40].

Characterization of sprouting fibers revealed the absence of $A \beta$ fiber sprouting as we never observed co-localization of VGLUT-1 (marker for myelinated afferents) with GAP-43. As the concept of direct activation of NS projection neurons upon non-nociceptive input requires sprouting of $A \beta$ fibers, our findings do not support this concept and provides additional evidence to more recent publications arguing against $A \beta$ fiber sprouting into the superficial layers [28,29,31]. We also did not observe sprouting of nonpeptidergic nociceptive fibers, evidenced by absent colocalization of IB4 and GAP-43. However, a strong colocalization was found for GAP-43 and CGRP, which indicated sprouting responses of peptidergic nociceptive afferents. These data may imply that sprouting of CGRP-positive fibers induce sensitization of the circuit, resulting in indirect activation of NS projection neurons upon non- 
nociceptive input as proposed to contribute to mechanical allodynia (Fig 8D). The current findings also provide further insight into the data presented by Hu and co-workers [6], who demonstrated a sprouting response of nociceptive afferents without investigating their exact nature. Additionally, these authors [6] showed that sprouting responses occur at spinal levels corresponding to L5 spinal nerve innervation levels. We confirmed this observation as we identified robust sprouting responses at the L5 and L4 spinal level, corresponding to the innervation level of the L5 spinal nerve [42]. This may imply that sprouting responses are specific for deafferentiated regions of the spinal cord. However, deafferentiation alone has been reported to be insufficient to induce sprouting responses [43]. This indicates that the creation of synaptic space is insufficient to induce primary afferent sprouting but possibly requires additional growth-inducing signals [43].

Sprouting of CGRP-positive fibers has notably been observed in the presence of NGF overexpression [10]. NGF is known to be released by microglia in the rat spinal cord [44]. In the present study, microglial activation was found to coincide with CGRP sprouting responses. We confirmed previous findings which show that microglia are activated at 5 days following L5 SNT [18], a response which could be partly inhibited in our study using i.t. injection of $10 \mu \mathrm{g}$ PPF. The concentration of i.t injected PPF in this study (i.e. $10 \mu \mathrm{g}$ ) was shown to effectively inhibit microglial iba-1 expression following L5 spinal nerve transection [20] and has anti-allodynic effects for at least 15 hours [45]. From this, we concluded that daily bolus injections of 10 $\mu \mathrm{g}$ of PPF as used in the present study are effective in inhibiting continuous processes like microglial activation. Indeed, PPF significantly attenuated microglial iba-1 expression, however, no reduction in sprouting responses was observed. Although microglial iba-1 expression was significantly reduced, expression is still $23 \%$ higher in the ipsilateral compared to the contralateral dorsal horn of PPF-treated L5 SNT animals. The lack of complete inhibition of microglial activation following nerve injury corresponds to earlier studies in which PPF (either intrathecally or intraperitoneally injected) was unable to completely inhibit microglial activation $[19,20,45]$. As we were unable to completely inhibit microglial responses, we cannot definitely conclude that microglial activation is unrelated to sprouting. The remaining fraction of activated microglia may explain the observed sprouting responses. While attenuation of microglial activation following PPF treatment did not result in a reduction of sprouting, a partly anti-allodynic effect could be observed. This anti-allodynic effect may be attributed to the well-known roles of activated microglia in symptoms of neuropathic pain $[46,47,48]$. Microglia are capable of releasing a number of substances such as pro-inflammatory cytokines and other neuro-active inflammatory mediators [49] that may increase neuronal excitability [50].

In conclusion, we provide evidence that nerve-injury induced static mechanical allodynia is paralleled by increases in cellular activation in specific spinal dorsal horn regions crucial in the development of neuropathic pain. This increased activation of dorsal horn neurons, which are likely to be part of a local circuit comprising PKCY-positive interneurons and NS projection neurons, may be mediated via sensitization due to sprouting of nociceptive primary afferents. This sensitization ultimately allows indirect transmission from non- 
nociceptive input towards NS projection neurons, thereby underlying mechanical allodynia observed following $L 5$ spinal nerve injury. Attenuation of microglial activation resulted in an anti-allodynic effect which, however, was not accompanied by a reduced sprouting response. The observed structural changes within the spinal dorsal horn circuit may explain the chronic nature of nerve injury-induced mechanical allodynia and the ineffectiveness of available anti-pain medication.

\section{Acknowledgements}

The authors wish to thank Ms. Marijke Lemmens for her assistance in the use of the stereologybased cell counting method and the confocal SI-SD system. Thanks to Ms. Liesbeth Knaepen for critically reading the manuscript. This work was financially supported by a grant from the Dutch Government (SENTERNOVEM grant \# IS 041064; to E.A.J.J. and M.A.E.M.), a grant from the Dutch Polymer Institute (DPI grant \# 608 to E.A.J.J., M.A.E.M and R.D.), and two Kootstra fellowships of the Maastricht University (to R.J.P.J. and R.D.). The SI-SD system was funded by grant no. 911-06-003 from the Medical Section of the Dutch Scientific Organization (NWO). The authors declare that there is no conflict of interest concerning this work.

\section{References}

1. Todd, A.J., Anatomy of primary afferents and projection neurones in the rat spinal dorsal horn with particular emphasis on substance P and the neurokinin 1 receptor. Exp Physiol, 2002. 87(2): p. 245-9.

2. Neumann, S., et al., Innocuous, not noxious, input activates PKCgamma interneurons of the spinal dorsal horn via myelinated afferent fibers. J Neurosci, 2008. 28(32): p. 7936-44.

3. Malmberg, A.B., et al., Preserved acute pain and reduced neuropathic pain in mice lacking PKCgamma. Science, 1997. 278(5336): p. 279-83.

4. Miraucourt, L.S., R. Dallel, and D.L. Voisin, Glycine inhibitory dysfunction turns touch into pain through PKCgamma interneurons. PLoS One, 2007. 2(11): p. e1116.

5. Miraucourt, L.S., et al., Glycine inhibitory dysfunction induces a selectively dynamic, morphine-resistant, and neurokinin 1 receptor-independent mechanical allodynia. J Neurosci, 2009. 29(8): p. 2519-27.

6. Hu, J., et al., Central sprouting of uninjured small fiber afferents in the adult rat spinal cord following spinal nerveligation. Eur J Neurosci, 2004. 20(7): p. 1705-12.

7. Lekan, H.A., S.M. Carlton, and R.E. Coggeshall, Sprouting of A beta fibers into lamina II of the rat dorsal horn in peripheral neuropathy. Neurosci Lett, 1996. 208(3): p. 147-50.

8. Shortland, P., E. Kinman, and C. Molander, Sprouting of A-fibre primary afferents into lamina II in two rat models of neuropathic pain. Eur J Pain, 1997. 1(3): p. 215-27.

9. Woolf, C.J., P. Shortland, and R.E. Coggeshall, Peripheral nerve injury triggers central sprouting of myelinated afferents. Nature, 1992. 355(6355): p. 75-8. 
10. Romero, M.I., et al., Extensive sprouting of sensory afferents and hyperalgesia induced by conditional expression of nerve growth factor in the adult spinal cord. J Neurosci, 2000. 20(12): p. 4435-45.

11. White, D.M., Neurotrophin-3 antisense oligonucleotide attenuates nerve injury-induced Abeta-fibre sprouting. Brain Res, 2000. 885(1): p. 79-86.

12. Molliver, D.C., et al., IB4-binding DRG neurons switch from NGF to GDNF dependence in early postnatal life. Neuron, 1997. 19(4): p. 849-61.

13. Bennett, D.L., et al., A distinct subgroup of small DRG cells express GDNF receptor components and GDNF is protective for these neurons after nerve injury. J Neurosci, 1998. 18(8): p. 3059-72.

14. Elkabes, S., L. Peng, and I.B. Black, Lipopolysaccharide differentially regulates microglial trk receptor and neurotrophin expression. J Neurosci Res, 1998. 54(1): p. 117-22.

15. Madiai, F., et al., Upregulation of FGF-2 in reactive spinal cord astrocytes following unilateral lumbar spinal nerveligation. Exp Brain Res, 2003. 148(3): p. 366-76.

16. Mele, T., M. Carman-Krzan, and D.M. Juric, Regulatory role of monoamine neurotransmitters in astrocytic NT-3 synthesis. Int J Dev Neurosci. 28(1): p. 13-9.

17. Rudge, J.S., et al., Expression of Ciliary Neurotrophic Factor and the Neurotrophins-Nerve Growth Factor, Brain-Derived Neurotrophic Factor and Neurotrophin 3-in Cultured Rat Hippocampal Astrocytes. Eur J Neurosci, 1992. 4(6): p. 459-471.

18. Romero-Sandoval, A., et al., A comparison of spinal Ibal and GFAP expression in rodent models of acute and chronic pain. Brain Res, 2008. 1219: p. 116-26.

19. Raghavendra, V., F. Tanga, and J.A. DeLeo, Inhibition of microglial activation attenuates the development but not existing hypersensitivity in a rat model of neuropathy. J Pharmacol Exp Ther, 2003. 306(2): p. 624-30.

20. Raghavendra, V., et al., Anti-hyperalgesic and morphine-sparing actions of propentofylline following peripheral nerve injury in rats: mechanistic implications of spinal glia and proinflammatory cytokines. Pain, 2003. 104(3): p. 655-64.

21. Chung, J.M., H.K. Kim, and K. Chung, Segmental spinal nerve ligation model of neuropathic pain. Methods Mol Med, 2004. 99: p. 35-45.

22. Zhang, E.T., et al., Nerve injury-induced tactile allodynia is present in the absence of FOS labeling in retrogradely labeled post-synaptic dorsal column neurons. Pain, 2007. 129(1-2): p. 143-54.

23. West, M.J., L. Slomianka, and H.J. Gundersen, Unbiased stereological estimation of the total number of neurons in thesubdivisions of the rat hippocampus using the optical fractionator. Anat Rec, 1991. 231(4): p. 482-97.

24. Schmitz, C. and P.R. Hof, Design-based stereology in neuroscience. Neuroscience, 2005. 130(4): p. 813-31.

25. Woolf, C.J., et al., Reorganization of central terminals of myelinated primary afferents in the rat dorsal horn following peripheral axotomy. J Comp Neurol, 1995. 360(1): p. 121-34.

26. Doubell, T.P., R.J. Mannion, and C.J. Woolf, Intact sciatic myelinated primary afferent terminals collaterally sprout in the adult rat dorsal horn following section of a neighbouring peripheral nerve. I Comp Neurol, 1997. 380(1): p. 95-104.

27. Nakamura, S. and R.R. Myers, Myelinated afferents sprout into lamina II of L3-5 dorsal horn following chronic constriction nerve injury in rats. Brain Res, 1999. 818(2): p. 285-90.

118 Page 
28. Bao, L., et al., Peripheral axotomy induces only very limited sprouting of coarse myelinated afferents into inner lamina ll of rat spinal cord. Eur J Neurosci, 2002. 16(2): p. 175-85.

29. Tong, Y.G., et al., Increased uptake and transport of cholera toxin B-subunit in dorsal root ganglion neurons after peripheral axotomy: possible implications for sensory sprouting. J Comp Neurol, 1999. 404(2): p. $143-58$.

30. Jancso, G., et al., Selective C-fiber deafferentation of the spinal dorsal horn prevents lesion-induced transganglionic transport of choleragenoid to the substantia gelatinosa in the rat. Neurosci Lett, 2004. 361(13): p. 204-7.

31. Hughes, D.I., et al., Lack of evidence for sprouting of Abeta afferents into the superficial laminas of the spinal cord dorsal horn after nerve section. J Neurosci, 2003. 23(29): p. 9491-9.

32. Woodbury, C.J., et al., Identity of myelinated cutaneous sensory neurons projecting to nocireceptive laminae following nerve injury in adult mice. J Comp Neurol, 2008. 508(3): p. 500-9.

33. Devor, M., Ectopic discharge in Abeta afferents as a source of neuropathic pain. Exp Brain Res, 2009. 196(1): p. 115-28.

34. Liu, X., et al., Spontaneous activity of axotomized afferent neurons after $L 5$ spinal nerve injury in rats. Pain, 2000. 84(2-3): p. 309-18.

35. Jang, J.H., et al., The role of uninjured C-afferents and injured afferents in the generation of mechanical hypersensitivity after partial peripheral nerve injury in the rat. Exp Neurol, 2007. 204(1): p. 288-98.

36. Li, Y., et al., Mechanical hyperalgesia after an L5 spinal nerve lesion in the rat is not dependent on input from injured nerve fibers. Pain, 2000. 85(3): p. 493-502.

37. Wall, P.D. and M. Gutnick, Ongoing activity in peripheral nerves: the physiology and pharmacology of impulses originating from a neuroma. Exp Neurol, 1974. 43(3): p. 580-93.

38. Amir, R., J.D. Kocsis, and M. Devor, Multiple interacting sites of ectopic spike electrogenesis in primary sensory neurons. J Neurosci, 2005. 25(10): p. 2576-85.

39. Wu, G., et al., Early onset of spontaneous activity in uninjured C-fiber nociceptors after injury to neighboring nerve fibers. J Neurosci, 2001. 21(8): p. RC140.

40. Baba, H., et al., Removal of GABAergic inhibition facilitates polysynaptic A fiber-mediated excitatory transmission to the superficial spinal dorsal horn. Mol Cell Neurosci, 2003. 24(3): p. 818-30.

41. Kohno, T., et al., Peripheral nerve injury alters excitatory synaptic transmission in lamina ll of the rat dorsal horn. J Physiol, 2003. 548(Pt 1): p. 131-8.

42. Shehab, S.A., Acute and chronic sectioning of fifth lumbar spinal nerve has equivalent effects on the primary afferents of sciatic nerve in rat spinal cord. J Comp Neurol, 2009. 517(4): p. 481-92.

43. Mannion, R.J., et al., Deafferentation is insufficient to induce sprouting of A-fibre central terminals in the rat dorsal horn. J Comp Neurol, 1998. 393(2): p. 135-44.

44. Krenz, N.R. and L.C. Weaver, Nerve growth factor in glia and inflammatory cells of the injured rat spinal cord. J Neurochem, 2000. 74(2): p. 730-9.

45. Sweitzer, S.M., P. Schubert, and J.A. DeLeo, Propentofylline, a glial modulating agent, exhibits antiallodynic properties in a rat model of neuropathic pain. J Pharmacol Exp Ther, 2001. 297(3): p. 1210-7.

46. Inoue, K. and M. Tsuda, Microglia and neuropathic pain. Glia, 2009. 57(14): p. 1469-79.

47. Suter, M.R., et al., Do glial cells control pain? Neuron Glia Biol, 2007. 3(3): p. 255-268. 
48. Tsuda, M., K. Inoue, and M.W. Salter, Neuropathic pain and spinal microglia: a big problem from molecules in "small" glia. Trends Neurosci, 2005. 28(2): p. 101-7.

49. Zhao, P., S.G. Waxman, and B.C. Hains, Extracellular signal-regulated kinase-regulated microglia-neuron signaling by prostaglandin E2 contributes to pain after spinal cord injury. J Neurosci, 2007. 27(9): p. 2357-68.

50. McMahon, S.B., W.B. Cafferty, and F. Marchand, Immune and glial cell factors as pain mediators and modulators. Exp Neurol, 2005. 192(2): p. 444-62. 


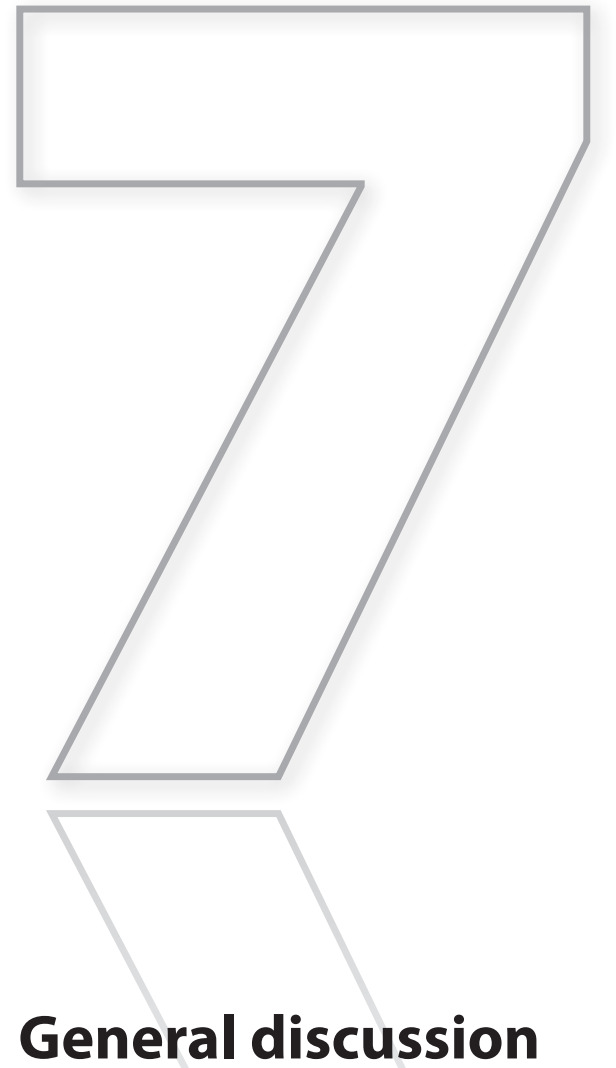




\section{Neuropathic pain: the clinical problem}

Despite the numerous pharmacological and non-pharmacological anti-pain strategies which exist to treat chronic neuropathic pain, still lots of patients cannot adequately be treated. Moreover, patients who do benefit from current anti-pain medication often suffer from severe side effects. The question is why these therapies lack efficacy in neuropathic pain patients. The rather low therapeutic effects of currently used pharmaco-therapies in treatment of neuropathic pain may partly be explained by the neuron-centric action of these drugs.

Clinically, chronic neuropathic pain can be defined as neuropathic pain persisting for at least three months [1]. The phases before this time point may be described as the early phase of neuropathic pain, in which mechanisms may be involved contributing to the transition from acute to chronic neuropathic pain.

Although minimal data exists on the total prevalence of neuropathic pain, studies have shown that about $8 \%$ of chronic pain in patients was of neuropathic origin, with greater severity of symptoms and greater impact on quality of life compared to patients suffering from other types of chronic pain [2,3,4]. More specifically, pain observed in 37\% of low back pain patients was predominantly neuropathic [5]. This high prevalence of neuropathic pain indicates the need for more efficient pharmacotherapies which (1) prevent the transition from acute to chronic pain or (2) reverse chronic neuropathic pain (i.e. neuropathic pain present for at least 3 months). Neuropathic pain may develop in different conditions, and adequate diagnosis is crucial to optimize the therapeutic approach [6]. Appropriate early diagnosis of neuropathic pain may aid in the application of effective treatments, ideally preventing the transition from acute to persistent, chronic neuropathic pain. The development of effective treatments to prevent the transition from acute to chronic pain require substantial knowledge into mechanisms involved in early phases of neuropathic pain, whereas insights into the mechanisms involved in chronic phases of neuropathic pain is crucial to develop drugs which may reverse neuropathic pain.

\section{What do we know and what knowledge is missing?}

Extensive research has been done investigating the mechanisms explaining the intractable nature of neuropathic pain. Current insights in the cellular and molecular mechanisms which underlie the intractable and chronic nature of neuropathic pain were reviewed in chapter 2 of this thesis. This thesis further describes mechanisms possibly involved in both the chronic and early phases of neuropathic pain, focusing on structural changes and glial responses in peripheral nerve injury-induced neuropathic pain. For this, two different animal models (sciatic nerve transection and L5 spinal nerve transection) were used which differ in the temporal occurrence of neuropathic pain symptoms.

Structural changes, including sprouting of primary afferents and synaptogenesis, have been described earlier in peripheral nerve injury models $[7,8,9,10,11]$. These structural 
changes are known to occur within the dorsal horn of the spinal cord, without further exploring the spatial aspect of these structural changes in detail. The dorsal horn of the rat spinal cord is divided in different laminae [12]. Herein, lamina II (i.e. substantia gelatinosa) was shown to be of crucial importance in neuropathic pain $[13,14]$. More specifically, a specific part of the lamina II comprises PKCY-positive, excitatory interneurons which have been shown to be pivotal in the development of neuropathic pain $[15,16]$. These PKC -positive interneurons are connected to nociception-specific projection neurons in lamina I, a circuit which normally inactive as it is under inhibitory control $[17,18]$. The specificity of the spinal nociceptive network illustrates that detailed spatial investigations of structural changes may provide important insights into the mechanisms underlying neuropathic pain.

Therefore, experiments are needed to assess whether synaptic plasticity is present in specific regions involved in the processing of nociceptive input. Detailed investigations in the spatial aspect of structural changes may be of utmost importance to link the outcome measures to the neuropathic pain symptoms. Therefore, we focused on synaptic plasticity within a specific layer of the dorsal horn, i.e. substantia gelatinosa.

As the location of primary afferent sprouting is also important to provide insights on how these structural changes may be involved in neuropathic pain, we investigated primary afferent sprouting within a spinal nociceptive network consisting of PKC $\mathrm{y}$-positive interneurons connected to nociception-specific projection neurons (located in most superficial layers of the dorsal spinal cord) $[16,17]$. Thus, sprouting responses within this nociceptive network may underlie sensitization of the circuit and subsequently mediate neuropathic pain. Although earlier studies have shown that fiber sprouting may occur in the setting of peripheral nerve injury, the nature of these sprouting responses needs further investigation. The first studies describing primary afferent sprouting following peripheral nerve injury report sprouting of myelinated, $A \beta$ afferents $[9,10]$. Additional studies reported nerve injury-induced sprouting of non-myelinated $A \delta$ and $C$ fibers [8]. However, these results were based on tracing procedures which do not provide clear evidence in the nature of sprouting fibers. Therefore, in chapter 6 , we combined markers for sprouting fibers with different primary afferent markers to clearly identify the nature of sprouting primary afferents.

Other possible mechanisms involved in neuropathic pain and its intractable nature may be mediated by microglia and/or astroglia. During the last decade, extensive research into the role of these glial cells in neuropathic pain has revealed their pro-nociceptive properties. Although knowledge on the role of microglia and astroglia in neuropathic pain is increasing, further insights in their exact role in neuropathic pain are needed to develop effective anti-pain therapies.

During early phases of neuropathic pain, microglia and astroglia display pro-nociceptive properties $[19,20]$. Whether these glia are also involved in mediating structural changes within in the spinal nociceptive network in the early phases of neuropathic pain is not known. Therefore, in the current thesis we investigated whether interference with microglia and astroglial activation influences early sprouting responses following peripheral nerve injury. Glia 
have also been shown to be activated in chronic phases of neuropathic pain [21]. However, their precise role in these chronic phases of neuropathic pain is not entirely clear. In this thesis, we investigated whether the pro-nociceptive role of glia during early phases of neuropathic pain is also observed in chronic neuropathic pain.

\section{Animal models of neuropathic pain}

\section{Which models to choose to study neuropathic pain mechanisms?}

This thesis describes (1) early (i.e. within the first weeks) and (2) chronic (i.e. at three months) nerve injury-induced neuroplasticity and glial responses in the spinal nociceptive network. In the present thesis, two different animal models were used which show either a quick onset of neuropathic pain symptoms or a persistency of such symptoms beyond three months.

The sciatic nerve transection model was selected to study mechanisms involved in the chronicity of neuropathic pain as this model displays mechanical hypersensitivity for at least 3 months. The assessment of neuropathy-associated pain symptoms in animals is mostly dependent on tests which rely on paw withdrawal reflexes to externally applied painful or non-painful stimuli. The von Frey method is an important tool to assess paw withdrawal reflexes towards an externally applied mechanical stimulus. As the sciatic nerve innervates a substantial number of hind limb muscles, transection of the sciatic nerve may result in severe motor impairments of the hind limb. These motor impairments may substantially interfere with paw withdrawal reflexes as elicited with the von Frey filaments. In this thesis, a detailed analysis of motor function was performed on rat with sciatic nerve transection.

The CatWalk method is an objective and automated method to assess static and dynamic changes in gait of experimental animals [22]. Stance and swing duration are parameters which indicate whether extensor and/or flexor hind limb muscles are functioning properly. A decade ago, Sherrington stated that the nociceptive withdrawal reflex reflects an activation of predominantly flexors and inhibition of extensors [23]. These observations led to the term "flexion reflex" when describing the nociceptive withdrawal reflex. Later, an elegant study showed that almost all hind limb flexor muscles are activated upon firm pressure and/or noxious mechanical stimulation of the hind paw [24]. Muscles responsible for withdrawal reflexes were plantar flexors of the digits, pronators of the paw, dorsiflexors and plantar flexors of the ankle, flexors of the knee and the hip and the adductors.

As observed with the CatWalk method, sciatic nerve transection resulted in increased swing duration whereas stance duration was decreased. Practically, this indicates that the animal is able to display perfect flexion of the hind limb muscles. Increased swing duration indicates that the animal's flexion period is longer in time than normal, most likely to avoid the injured paw. Speed of gait may have a strong influence on the parameters 
stance and swing duration but was not affected by sciatic nerve transection. This finding indicates reliable assessment of both swing and stance duration and also shows that hind limb muscles function is not severely affected following sciatic nerve transection.

Thus, based on the results obtained from chapter 3 we conclude that changes in motor-related parameters following sciatic nerve transection as measured with the CatWalk method do not interfere with paw withdrawal reflexes. Therefore, outcome measures of the von Frey method to assess mechanical hypersensitivity are reliable, which makes the sciatic nerve transection model suitable to investigate mechanisms underlying chronic neuropathic pain.

The L5 spinal nerve transection model was selected to study mechanisms involved in the the intractable nature of neuropathic pain and initiating the neuroplastic changes seen at chronic stages of neuropathic pain. The quick onset of mechanical hypersensitivity and glial responses present in this model make it possible to study early neuropathic pain mechanisms [25].

As in this model only the L5 spinal nerve was transected, only minor effects on motor function were observed including mild inversion of the foot with slightly ventroflexed toes [26]. However, as these minor changes do not interfere with paw withdrawal reflex function, reliable assessment of mechanical hypersensitivity as assessed with the von Frey method is possible. It has been shown that the intact $L 4$ spinal nerve is important for motor functions of the related hind paw, and thus injury to this specific nerve resulted in complete paralysis of the corresponding hind paw [26].

Investigating neuroplasticity and glial responses in a single animal model which displays both early onset and chronic neuropathic pain symptoms (i.e at least 3 months) would be optimal. Animal models which show an early onset and late presence of neuropathic pain symptoms do exist, although these have their limitations. Partial sciatic nerve ligation, in which $1 / 3$ to $1 / 2$ of the sciatic nerve is ligated, results in immediate onset (i.e. from 1 hour post-injury) of neuropathic pain symptoms which persist for at least 7 months post-injury [27]. However, at 7 months following initial injury the original study of Seltzer and coworkers only reports the presence of thermal hypersensitivity without investigating mechanical hypersensitivity at that time point [27]. Others reported that mechanical hypersensitivity to von Frey filaments resolved at 7 weeks post-injury in the partial sciatic nerve ligation model [28]. Moreover, the reliability of the extent of the injury limits the use of this animal model since outcome measures of behavioral pain tests may vary between individual animals. The same argument holds for the chronic constriction model, in which 4 chromic gut ligatures are loosely tied around the sciatic nerve at mid-thigh level [29].

An animal model which displays both early (i.e. 1 day post-injury) and late (i.e. 220 days post-injury) neuropathic pain symptoms is the spared nerve injury model, in which the common peroneal and tibial nerves are ligated and sectioned [30]. Variability in both surgery procedure and in outcome measures of the von Frey test are low, thereby making this neuropathic pain model suitable to investigate mechanisms involved in both early and late phase of neuropathic pain. Thus, the spared nerve injury model may be appropriate for future studies regarding the relationship between early sprouting responses and late synaptic changes. 


\section{Structural changes in neuropathic pain: does it explain the chronic and intractable nature?}

Based on the outcome of the study described in chapter 3, the sciatic nerve transection model was selected to investigate synaptic plasticity within the nociceptive network in chronic stages of neuropathic pain. An increase in the number of large-sized synapses in the substantia gelatinosa possibly indicates an increase in synaptic strength and efficacy, thereby contributing to the mechanical hypersensitivity observed following sciatic nerve transection. Neuronal plasticity in terms of altered connectivity has been suggested to underlie the intractable nature of neuropathic pain [31]. The relationship between synaptic plasticity and neuropathic pain has been investigated in term of quantifying the level of synaptic markers. More specifically, the pre-synaptic vesicle protein synaptophysin was shown to be upregulated in the ipsilateral dorsal horn following unilateral chronic constriction injury [7]. The increased levels of synaptophysin paralleled the thermal hyperalgesia, not mechanical allodynia. Remarkably, synaptophysin levels returned to levels comparable to baseline levels at 21 days post-injury. These results imply that an increase in synaptophysin represents structural synaptic changes during the early phases of neuropathic pain. However, no insights were provided in the spatial aspect of synaptophysin levels.

Therefore, we investigated synaptic plasticity in the substantia gelatinosa by measuring immunoreactivity of synaptophysin protein levels. Chapter 4 describes that the observed synaptic plasticity may occur according to Hebb's law, which states that synapses enlarge due to repetitive firing of presynaptic cells [32]. Although this repetitive firing may originate from neuroma tissue present at the injury site of the transected sciatic nerve, other factors may be involved in the observed chronic synaptic plasticity. As an example, astroglia have been shown to be intimately involved in synaptic plasticity. Protoplasmic astroglia, found in gray matter, ensheate synapses and thereby control neuronal pre-and postsynaptic signaling [33]. It has been shown that astroglia are crucial in the formation of functional synapses between cultured retinal ganglion cells [34]. Moreover, astroglia control total number of synapses formed and greatly influence pre-and postsynaptic signaling [35]. Suggested astroglia-associated factors involved in this synaptic plasticity are thrombospondins [36] and cholesterol [37].

Although astroglia may be indirectly involved in synaptic plasticity and thus may explain the chronic nature of neuropathic pain, the results presented in chapter 5 point towards an anti-nociceptive role of astroglia. At three months following peripheral nerve injury, astroglia were found to be activated within the substantia gelatinosa and were shown to be negatively correlated with the degree of mechanical allodynia. This indicates that astroglia seem to have anti-nociceptive properties, which is in contrast to the well-accepted idea of astroglia as contributors to the chronicity of neuropathic pain [19]. At the same time, microglia were not activated and, thus, not associated with chronic stages of nerve injury-induced neuropathic pain. 
In contrast, chapter 6 describes that microglia, and not astroglia, are activated at early phases of neuropathic pain. Here, activated microglia display pro-nociceptive properties since inhibition of microglia at early stages of neuropathic pain resulted in attenuated mechanical hypersensitivity. Moreover, we hypothesized that activated glia are involved in sprouting responses within the spinal nociceptive network in early phases of neuropathic pain. Therefore, sprouting responses were investigated by measuring immunoreactivity of growth-associated protein-43 (GAP-43). We observed robust fiber sprouting within the nociceptive network in the early phases of neuropathic pain, i.e. from 5 to 10 days post-injury. Sprouting fibers were of nociceptive nature and possible contributed to the sensitization and ultimately to the activation of the local circuit comprising PKCy-positive interneurons to nociception-specific projection neurons. This activation may underlie the mechanical hypersensitivity observed following L5 spinal nerve transection.

We used GAP-43 as a marker for fiber sprouting, however, GAP-43 is not solely present in growth cones of regenerating fibers but may also be expressed in astroglia [38]. Although observed in vitro, we (unpublished data) and others [39] have shown that in vivo astroglia are devoid of GAP-43. In contrast, Schwann cells present in the peripheral nerve axon do show GAP-43 expression following crush injury of the sciatic nerve [40].

We investigated the nature of sprouting fibers, peptidergic, CGRP-positive fibers display robust sprouting responses following L5 spinal nerve transection. This finding might have important implications for future therapeutic approaches. As an example, NGF is known to induce sprouting responses of CGRP-positive fibers and anti-NGF treatment thus results in a significant reduction of nociceptive fiber sprouting [41]. Moreover, capsaicin application may result in a degeneration of C fibers, which include CGRP-positive fibers, and thus may counteract the effect of the observed sprouting [42]. Capsaicin patches applied to the skin are currently used to treat neuropathic pain symptoms and were proven to provide pain relief for about 3 months after application of the patch [43].

An important question remains whether the observed sprouting fibers are injured or uninjured primary afferents, which may affect the choice of a specific therapeutic approach. Topical applied treatments would not interfere with central sprouting responses of injured fibers as the connection between periphery and spinal cord is lost. It is known that central axon terminals withdraw a short distance from their spinal cord target following peripheral nerve injury and subsequently regrow [44]. As these fibers regrow, they may establish "wrong" connections, thereby resulting in sensitization of spinal nociceptive circuit and ultimately resulting in neuropathic pain symptoms. Alternatively, recent studies revealed the importance of uninjured, L4 fiber sprouting into spinal regions denervated by L5 spinal nerve ligation [8]. Based on the results in this thesis, it remains to be elucidated whether injured or uninjured nociceptive primary afferents display increased levels of GAP-43 and thus sprouting responses. 


\section{The relationship between fiber sprouting and synaptic plasticity in neuropathic pain}

Important indications whether fiber sprouting is a prerequisite for synaptic plasticity may be based on the temporal occurrence of both processes. Sprouting responses, quantified by measuring GAP-43 immunoreactivity, were first observed at 5 days post-operative. At 21 days following injury, levels of GAP-43 immunoreactivity were decreased, returning to levels comparative to sham-operated animals. Development studies have shown that GAP43 expression declines once axons reach their target [45]. This may indicate that at 21 days following injury, sprouting fibers have reached their target and start to form functional synapses. Once functional synapses are formed, further synaptic plasticity like an increase in strength and efficacy, may occur resulting in an increase in synaptic size. Moreover, other studies have provided evidence that GAP-43 is particularly involved in the initial, active phase of neurite outgrowth [46].

In early phases of neuropathic pain, we observed sprouting of nociceptive, CGRP-positive fibers. In chronic phases of neuropathic pain, the nature of the large-sized synapses in the substantia gelatinosa was not investigated. Therefore, it remains unclear whether sprouting of CGRP-positive fibers results in the formation of CGRP-positive synapses at later time points. The fact that both CGRP-positive fiber sprouting and synaptic plasticity were observed in the same spinal layer might indicate that the large-sized synapses in chronic phases of neuropathic pain are also CGRP-positive. Further studies including double immunohistochemistry for synaptophysin and CGRP may provide important insights in this issue.

It should be emphasized that nociceptive fiber sprouting and synaptic plasticity, were investigated using two different animal models for neuropathic pain. These models significantly differ in the extent of the lesion to the sciatic nerve. This implies that spinal segments innervated by the sciatic nerve are more severely affected in case of transection of the sciatic nerve compared to transection of just one spinal nerve which is part of the sciatic nerve. Thus, the investigated spinal segments (L3-L5) receive input from either damaged fibers alone (in case of sciatic nerve transection) or overlapping input from both injured $L 5$ afferents as well as from uninjured $L 4$ afferents (in case of $L 5$ spinal nerve transection). This might have important implications whether sprouting responses and/or synaptic plasticity occurs or not and if so, when they occur.

Another difference noted between different animal models is the peripheral nerve injury-induced temporal expression of GAP-43 in the dorsal spinal cord. Sciatic nerve transection or sciatic nerve crush result in increased level of GAP-43 for several weeks post-injury [47], whereas we have shown that GAP-43 is only increased for about 10 following L5 spinal nerve transection. It has also been shown that an increase in GAP-43 expression is dependent on the site of axotomy relative to the soma $[48,49]$. The sites of axotomy differ largely in the models used in our studies and may thus explain possible differences in sprouting responses. 
The type of peripheral nerve injury may also influence the temporal activation patterns of microglia and astroglia. Although not investigated in the experiments described in this thesis, it is possible that microglia are activated in early phases in the sciatic nerve transection model and thereby contributing to sprouting responses. Another important issue is how to define glial responses. In the current thesis, microglia and astroglia activation are defined by an increased immunoreactivity of OX-42/iba-1 and GFAP, respectively. However, increased immunoreactivity of these markers only reflects morphological changes and do not give insights in functional changes of microglia and astroglia.

\section{The relationship between structural changes and glial responses in neuropathic pain}

Structural changes, including fiber sprouting and synaptic plasticity, may be mediated by growth factors like nerve growth factor (NGF) or brain-derived nerve growth factor (BDNF) [52,53]. Microglia are known sources of both NGF and BDNF [50,51]. In the setting of experimental ischemia in rat, inhibition of microglia activation resulted in reduced levels of BDNF and remarkably also in reduced levels of GAP-43 and synaptophysin [53]. Additionally, mutation of a protein crucial for microglia activation (KARAP/DAP12) resulted in significant alterations in synaptic function and plasticity during perinatal stages [54]. Altogether, these findings indicate that microglia play a prominent role in mediating structural changes in the injured CNS.

Besides microglia, synaptogenesis and synaptic strengthening may be regulated by astroglia. L5 spinal nerve transection results in a delayed astroglial activation (i.e. from 7 days post-injury onwards) [25]. As mentioned before, astroglia are intimately involved in controlling the number and functionality of synapses [34,35]. Thus from 7 days following L5 spinal nerve transection, astroglia may aid in the development of new synapses in the spinal nociceptive network. At late phase of neuropathic pain (i.e. 3 months post-injury), astroglia may be more involved in controlling pathologically enhanced neuronal signaling. Once new synapses are established, by microglia in early phases and by astroglia in the following phase, a negative feedback mechanism is created in which astroglia suppress synaptic activity and thereby attenuate chronic neuropathic pain.

Since these mechanisms are rather suggestive, additional experiments are needed to clarify the role of both microglia and astroglia in mediating fiber sprouting and/or synaptic plasticity in neuropathic pain. 


\section{Back to the clinical problem}

The results presented in this thesis point towards structural changes within the spinal nociceptive network in early and chronic neuropathic pain. In early phases of neuropathic pain, sprouting of CGRP-positive fibers was observed and was associated with increased cellular activation within the spinal nociceptive network. In chronic neuropathic pain, synaptic plasticity was observed in the spinal nociceptive network which may reflect an activity-dependent increase in synaptic size and strength.

These findings highlight the importance of early diagnosis of neuropathic pain. As early diagnosis may imply early treatment, therapies inhibiting sprouting responses in early phases of neuropathic pain may prevent the transition from acute to chronic pain. Possible treatment approaches may interfere directly with the process of sprouting. On the other hand, nonneuronal cells possibly involved in initiation of sprouting responses may represent promising therapeutic targets. In this thesis, we hypothesized that microglia may trigger nociceptive fiber sprouting by the release of growth factors. However, based on the findings described in chapter 6 , no definite conclusion can be made on the role of glia in nociceptive fiber sprouting.

Current pharmacotherapies to treat patient suffering from chronic neuropathic pain display relative poor responses and severe side effect. The lack of efficiency of today's antipain medication may be explained by the excess of excitatory input into the spinal nociceptive network. Synaptic plasticity, in this thesis evidenced by an increase in size of synapses, may reflect an increase in strength and efficacy of synaptic signaling. This excess of excitatory input can be counteracted by (1) a higher dose of currently available anti-pain medication or (2) new, more efficient drugs. Higher doses of current anti-pain therapies render severe side effects. Hence, drug development may be focused on interference with strengthening, and thus increases in size, of synapses within the spinal nociceptive network.

At the same time, we have shown that astroglia may have anti-nociceptive properties in chronic neuropathic pain. From this perspective, the use of glial inhibitors in patients suffering from chronic neuropathic pain should be approached with great caution.

\section{Future perspectives}

Inhibition of structural changes during early phases of neuropathic pain may inhibit longterm synaptic plasticity and thus attenuate chronic neuropathic pain. As shown in chapter 6 , increased levels of GAP-43 indicate sprouting responses during the first days following nerve injury. Interference with GAP-43 protein expression may results in impaired sprouting responses and thus reduced synaptic plasticity. Ultimately, this would prevent neuropathic pain to become chronic. Experimentally, inhibition of GAP-43 by antisense oligonucleotides or anti-GAP-43 antibodies resulted in impaired neuritogenesis, characterized by reduced growth cone size and poor growth cone adhesion $[55,56]$. In contrast, overexpression of GAP43 in adult transgenic mice is paralleled by a greatly potentiated sciatic nerve crush induced- 
sprouting of nerve fibers [57]. Additional studies are required to assess the functional and sensory implications of possible treatments interfering with early GAP-43 expression to inhibit sprouting responses during early phases of neuropathic pain.

The cell adhesion molecule (CAM) L1 is known to be important in axonal outgrowth as well as synapse formation [58,59]. L1 knock-out mice display less CGRP-positive fiber sprouting in the lumbar spinal cord in the setting of spinal cord injury when compared to their wildtype littermates [60]. Moreover, animals lacking the L1 molecule recovered from heat hyperalgesia induced upon spinal cord injury. These findings indicate that therapies interfering with L1 adhesion molecule might reduce early fiber sprouting following peripheral nerve injury and thereby attenuate neuropathic pain symptoms.

In this thesis, we described a sprouting response of nociceptive, CGRP-positive fibers. Once sprouting responses are initiated, early application of drugs which induce degeneration of nociceptive C-fibers, for example by topical application of high levels of capsaicin, may provide adequate pain relief. Topical application of capsaicin at early time points possibly prevents nociceptive fiber sprouting and thus the transition from acute to chronic pain. To prevent the initiation of sprouting responses, well-timed interference with growth-promoting factor like NGF may have satisfactorily effects [68].

However, the precise role of early fiber sprouting responses in neuropathy-induced hypersensitivity remains still poorly described. Although nociceptive fiber sprouting occurs during early phases of neuropathic pain in the $L 5$ spinal nerve transection model (chapter 6), animals display normal sensitivity to mechanical stimulation at 2 months following injury [61]. This again indicates that the link between sprouting responses during early phases of neuropathic pain and synaptic plasticity during chronic neuropathic pain needs to be further investigated.

During the last decade, it has become increasingly clear that microglia and astroglia are intimately involved in the development and early maintenance (i.e. about 3 weeks postinjury) of neuropathic pain $[19,62,63,64,65,66]$. Chronic constriction injury of the sciatic nerve results in robust activation of astroglia in the lumbar spinal cord at 10 days post-injury, in which the level of astroglial activation was correlated with the degree of neuropathic pain [67]. Moreover, L5/L6 spinal nerve ligated animals treated with GFAP antisense at 6 weeks post-injury displayed clear anti-allodynic effects, indicating that astroglia also display pronociceptive roles in maintenance of neuropathic pain [61].

In contrast to the concept that astroglia are crucial in the maintenance of neuropathic pain, we suggest that astroglia may display anti-nociceptive properties in chronic stages of neuropathic pain, based on the results presented in chapter 5 in this thesis. Here, we showed that an increase in astroglial activation was associated with a decrease in mechanical hypersensitivity following sciatic nerve transection. These findings may have important consequences for future therapeutic strategies and warrant for the use of glial inhibitors to attack established, chronic neuropathic pain. Although the effects of the glial modulatory drug AV411 on neuropathic pain is currently being tested in clinical trials, we propose that further research in the timing of the application of glial inhibitors is needed. 


\section{Conclusion}

Altogether, these findings indicate that the timing of application of anti-pain therapies is of utmost importance. Whereas inhibition of glia in early phases of neuropathic pain may be a good option, inhibition of astroglia in chronic phases may result in even more pain. We observed that the early phase of neuropathic pain is accompanied with fiber sprouting, possibly converting acute pain into chronic pain. This implies that Inhibition of early sprouting responses may prevent neuropathic pain to become chronic. Herein, early diagnosis of neuropathic pain is essential for adequate early treatments. In chronic phases of neuropathic pain, synaptic plasticity in terms of an activity-dependent increase in synaptic size and strength within the spinal nociceptive network may underlie an excess of excitatory input which cannot be adequately counteracted with current anti-pain medicine. This may explain the intractable nature of chronic neuropathic pain. Further research is needed to unravel the link between early and chronic peripheral nerve injury-induced structural changes, ultimately resulting in adequate anti-pain medication which deals with the intractable nature of chronic neuropathic pain.

\section{References}

1. Apkarian, A.V., M.N. Baliki, and P.Y. Geha, Towards a theory of chronic pain. Prog Neurobiol, 2009. 87(2): p. $81-97$.

2. Torrance, N., et al., The epidemiology of chronic pain of predominantly neuropathic origin. Results from a general population survey. J Pain, 2006. 7(4): p. 281-9.

3. Bouhassira, D., et al., Prevalence of chronic pain with neuropathic characteristics in the general population. Pain, 2008. 136(3): p. 380-7.

4. Smith, B.H., et al., Health and quality of life associated with chronic pain of predominantly neuropathic origin in the community. Clin J Pain, 2007. 23(2): p. 143-9.

5. Freynhagen, R., et al., painDETECT: a new screening questionnaire to identify neuropathic components in patients with back pain. Curr Med Res Opin, 2006. 22(10): p. 1911-20.

6. Haanpaa, M.L., et al., Assessment of neuropathic pain in primary care. Am J Med, 2009. 122 (10 Suppl): p. S13-21.

7. Chou, A.K., et al., Altered synaptophysin expression in the rat spinal cord after chronic constriction injury of sciatic nerve. Neurosci Lett, 2002. 333(3): p. 155-8.

8. Hu, J., et al., Central sprouting of uninjured small fiber afferents in the adult rat spinal cord following spinal nerve ligation. Eur J Neurosci, 2004. 20(7): p. 1705-12.

9. Woolf, C.J., P. Shortland, and R.E. Coggeshall, Peripheral nerve injury triggers central sprouting of myelinated afferents. Nature, 1992. 355(6355): p. 75-8.

10. Woolf, C.J., et al., Reorganization of central terminals of myelinated primary afferents in the rat dorsal horn following peripheral axotomy. J Comp Neurol, 1995. 360(1): p. 121-34. 
11. Lekan, H.A., S.M. Carlton, and R.E. Coggeshall, Sprouting of A beta fibers into lamina ll of the rat dorsal horn in peripheral neuropathy. Neurosci Lett, 1996. 208(3): p. 147-50.

12. Molander, C., Grant, G., Spinal Cord Cytoarchitecture. Second ed. The Nervous System. 1995, San Diego: Academic Press.

13. Baba, H., T.P. Doubell, and C.J. Woolf, Peripheral inflammation facilitates Abeta fiber-mediated synaptic input to the substantia gelatinosa of the adult rat spinal cord. J Neurosci, 1999. 19(2): p. 859-67.

14. Balasubramanyan, S., et al., Sciatic chronic constriction injury produces cell-type-specific changes in the electrophysiological properties of rat substantia gelatinosa neurons. J Neurophysiol, 2006. 96(2): p. 57990.

15. Malmberg, A.B., et al., Preserved acute pain and reduced neuropathic pain in mice lacking PKCgamma. Science, 1997. 278(5336): p. 279-83.

16. Neumann, S., et al., Innocuous, not noxious, input activates PKCgamma interneurons of the spinal dorsal horn via myelinated afferent fibers. J Neurosci, 2008. 28(32): p. 7936-44.

17. Miraucourt, L.S., R. Dallel, and D.L. Voisin, Glycine inhibitory dysfunction turns touch into pain through PKCgamma interneurons. PLoS One, 2007. 2(11): p. e1116.

18. Miraucourt, L.S., et al., Glycine inhibitory dysfunction induces a selectively dynamic, morphine-resistant, and neurokinin 1 receptor-independent mechanical allodynia. J Neurosci, 2009. 29(8): p. 2519-27.

19. Watkins, L.R., E.D. Milligan, and S.F. Maier, Glial activation: a driving force for pathological pain. Trends Neurosci, 2001. 24(8): p. 450-5.

20. McMahon, S.B., W.B. Cafferty, and F. Marchand, Immune and glial cell factors as pain mediators and modulators. Exp Neurol, 2005. 192(2): p. 444-62.

21. Coyle, D.E., Partial peripheral nerve injury leads to activation of astroglia and microglia which parallels the development of allodynic behavior. Glia, 1998. 23(1): p. 75-83.

22. Hamers, F.P., et al., Automated quantitative gait analysis during overground locomotion in the rat: its application to spinal cord contusion and transection injuries. J Neurotrauma, 2001. 18(2): p. 187-201.

23. Sherrington, C.S., Flexion-reflex of the limb, crossed extension-reflex, and reflex stepping and standing. J Physiol, 1910. 40(1-2): p. 28-121.

24. Schouenborg, J. and J. Kalliomaki, Functional organization of the nociceptive withdrawal reflexes. I. Activation of hindlimb muscles in the rat. Exp Brain Res, 1990. 83(1): p. 67-78.

25. Romero-Sandoval, A., et al., A comparison of spinal Iba1 and GFAP expression in rodent models of acute and chronic pain. Brain Res, 2008. 1219: p. 116-26.

26. Chung, J.M., H.K. Kim, and K. Chung, Segmental spinal nerve ligation model of neuropathic pain. Methods Mol Med, 2004. 99: p. 35-45.

27. Seltzer, Z., R. Dubner, and Y. Shir, A novel behavioral model of neuropathic pain disorders produced in rats by partial sciatic nerve injury. Pain, 1990. 43(2): p. 205-18.

28. Dowdall, T., I. Robinson, and T.F. Meert, Comparison of five different rat models of peripheral nerve injury. Pharmacol Biochem Behav, 2005. 80(1): p. 93-108.

29. Bennett, G.J. and Y.K. Xie, A peripheral mononeuropathy in rat that produces disorders of pain sensation like those seen in man. Pain, 1988. 33(1): p. 87-107. 
30. Decosterd, I. and C.J. Woolf, Spared nerve injury: an animal model of persistent peripheral neuropathic pain. Pain, 2000. 87(2): p. 149-58.

31. Woolf, C.J. and M.W. Salter, Neuronal plasticity: increasing the gain in pain. Science, 2000. 288(5472): p. $1765-9$.

32. Hebb, D.O., The organization of Behavior. 1949, New York: Wiley.

33. Barres, B.A., The mystery and magic of glia: a perspective on their roles in health and disease. Neuron, 2008. 60(3): p. 430-40.

34. Pfrieger, F.W. and B.A. Barres, Synaptic efficacy enhanced by glial cells in vitro. Science, 1997. 277(5332): p. 1684-7.

35. Ullian, E.M., et al., Control of synapse number by glia. Science, 2001. 291 (5504): p. 657-61.

36. Christopherson, K.S., et al., Thrombospondins are astrocyte-secreted proteins that promote CNS synaptogenesis. Cell, 2005. 120(3): p. 421-33.

37. Goritz, C., et al., Role of glia-derived cholesterol in synaptogenesis: new revelations in the synapse-glia affair. J Physiol Paris, 2002. 96(3-4): p. 257-63.

38. Vitkovic, L., et al., The 43-kDa neuronal growth-associated protein (GAP-43) is present in plasma membranes of rat astrocytes. Proc Natl Acad Sci U S A, 1988. 85(21): p. 8296-300.

39. Coggeshall, R.E., M.L. Reynolds, and C.J. Woolf, Distribution of the growth associated protein GAP-43 in the central processes of axotomized primary afferents in the adult rat spinal cord; presence of growth conelike structures. Neurosci Lett, 1991. 131(1): p. 37-41.

40. Plantinga, L.C., et al., The expression of B-50/GAP-43 in Schwann cells is upregulated in degenerating peripheral nerve stumps following nerve injury. Brain Res, 1993. 602(1): p. 69-76.

41. Christensen, M.D. and C.E. Hulsebosch, Spinal cord injury and anti-NGF treatment results in changes in CGRP density and distribution in the dorsal horn in the rat. Exp Neurol, 1997. 147(2): p. 463-75.

42. Lynn, B., Capsaicin: actions on nociceptive C-fibres and therapeutic potential. Pain, 1990. 41 (1): p. 61-9.

43. Backonja, M., et al., NGX-4010, a high-concentration capsaicin patch, for the treatment of postherpetic neuralgia: a randomised, double-blind study. Lancet Neurol, 2008. 7(12): p. 1106-12.

44. Knyihar-Csillik, E., P. Rakic, and B. Csillik, Fine structure of growth cones in the upper dorsal horn of the adult primate spinal cord in the course of reactive synapto-neogenesis. Cell Tissue Res, 1985. 239(3): p. 633-41.

45. Fitzgerald, M., M.L. Reynolds, and LI. Benowitz, GAP-43 expression in the developing rat lumbar spinal cord. Neuroscience, 1991. 41 (1): p. 187-99.

46. Shea, T.B., et al., Phospholipid-mediated delivery of anti-GAP-43 antibodies into neuroblastoma cells prevents neuritogenesis. J Neurosci, 1991. 11(6): p. 1685-90.

47. Woolf, C.J., et al., The growth-associated protein GAP-43 appears in dorsal root ganglion cells and in the dorsal horn of the rat spinal cord following peripheral nerve injury. Neuroscience, 1990. 34(2): p. 465-78.

48. Doster, S.K., et al., Expression of the growth-associated protein GAP-43 in adult rat retinal ganglion cells following axon injury. Neuron, 1991. 6(4): p. 635-47.

49. Tetzlaff, W., et al., Response of facial and rubrospinal neurons to axotomy: changes in mRNA expression for cytoskeletal proteins and GAP-43. J Neurosci, 1991. 11(8): p. 2528-44. 
50. Krenz, N.R. and L.C. Weaver, Nerve growth factor in glia and inflammatory cells of the injured rat spinal cord. J Neurochem, 2000. 74(2): p. 730-9.

51. Coull, J.A., et al., BDNF from microglia causes the shift in neuronal anion gradient underlying neuropathic pain. Nature, 2005. 438(7070): p. 1017-21.

52. Romero, M.I., et al., Extensive sprouting of sensory afferents and hyperalgesia induced by conditional expression of nerve growth factor in the adult spinal cord. J Neurosci, 2000. 20(12): p. 4435-45.

53. Madinier, A., et al., Microglial involvement in neuroplastic changes following focal brain ischemia in rats. PLoS One, 2009. 4(12): p. e8101.

54. Bessis, A., et al., Microglial control of neuronal death and synaptic properties. Glia, 2007. 55(3): p. 233-8.

55. Shea, T.B., Delivery of anti-GAP-43 antibodies into neuroblastoma cells reduces growth cone size. Biochem Biophys Res Commun, 1994. 203(1): p. 459-64.

56. Aigner, L. and P. Caroni, Absence of persistent spreading, branching, and adhesion in GAP-43-depleted growth cones. J Cell Biol, 1995. 128(4): p. 647-60.

57. Aigner, L., et al., Overexpression of the neural growth-associated protein GAP-43 induces nerve sprouting in the adult nervous system of transgenic mice. Cell, 1995. 83(2): p. 269-78.

58. Kamiguchi, H., The mechanism of axon growth: what we have learned from the cell adhesion molecule L1. Mol Neurobiol, 2003. 28(3): p. 219-28.

59. Godenschwege, T.A., et al., A conserved role for Drosophila Neuroglian and human L1-CAM in centralsynapse formation. Curr Biol, 2006. 16(1): p. 12-23.

60. Hoschouer, E.L., F.Q. Yin, and L.B. Jakeman, L1 cell adhesion molecule is essential for the maintenance of hyperalgesia after spinal cord injury. Exp Neurol, 2009. 216(1): p. 22-34.

61. Kim, D.S., et al., Profiling of dynamically changed gene expression in dorsal root ganglia post peripheral nerve injury and a critical role of injury-induced glial fibrillary acidic protein in maintenance of pain behaviors [corrected]. Pain, 2009. 143(1-2): p. 114-22.

62. Scholz, J. and C.J. Woolf, The neuropathic pain triad: neurons, immune cells and glia. Nat Neurosci, 2007. 10(11): p. 1361-8.

63. Raghavendra, V., F. Tanga, and J.A. DeLeo, Inhibition of microglial activation attenuates the development but not existing hypersensitivity in a rat model of neuropathy. J Pharmacol Exp Ther, 2003. 306(2): p. 624-30.

64. Zhuang, Z.Y., et al., A peptide c-Jun N-terminal kinase (JNK) inhibitor blocks mechanical allodynia after spinal nerve ligation: respective roles of JNK activation in primary sensory neurons and spinal astrocytes for neuropathic pain development and maintenance. J Neurosci, 2006. 26(13): p. 3551-60.

65. Ji, R.R., et al., Possible role of spinal astrocytes in maintaining chronic pain sensitization: review of current evidence with focus on bFGF/JNK pathway. Neuron Glia Biol, 2006. 2(4): p. 259-269.

66. Kozai, T., et al., Tissue type plasminogen activator induced in rat dorsal horn astrocytes contributes to mechanical hypersensitivity following dorsal root injury. Glia, 2007. 55(6): p. 595-603.

67. Garrison, C.J., et al., Staining of glial fibrillary acidic protein (GFAP) in lumbar spinal cord increases following a sciatic nerve constriction injury. Brain Res, 1991. 565(1): p. 1-7.

68. Hefti, F.F., et al., Novel class of pain drugs based on antagonism of NGF. Trends Pharmacol Sci, 2006. 27(2): p. 85-91. 



\section{Summary}

Chapter 1 provides a brief overview of the clinical problem of neuropathic pain, a short description of the animals used in this thesis and states the research questions and aims of the thesis.

In Chapter 2, the current literature regarding mechanisms underlying neuropathic pain is reviewed. Special attention is given to the neuronal and non-neuronal factors contributing to the intractable and chronic nature of neuropathic pain.

Chapter 3 describes the extent of persistent deficits of motor functions associated with complete sciatic nerve transection. As a severe degree of motor impairments may strongly interfere with outcome measures of pain-assessment test in rats, which heavily depend on paw withdrawal reflexes, the findings in this chapter may be of particular interest for the interpretation of pain data obtained using this animal model of chronic neuropathic pain. It is concluded that the observed degree of changes in motor functions following sciatic nerve transection do not suggest any motor deficits that may interfere with paw withdrawal reflexes. Thus, the sciatic nerve transection model is a reliable model to assess neuropathic pain symptoms and can thus be used to investigate mechanisms underlying the chronic nature of neuropathic pain.

Chapter 4 describes the synaptic changes present in chronic neuropathic pain. Sciatic nerve transection resulted in chronic neuropathic pain which persisted at least 3 months following injury. At this time point, synaptic changes were assessed by measuring differences in largesized synapses. Immunohistochemical staining for the pre-synaptic protein synaptophysin provided evidence that the number of large-sized synapses were increased in the ipsilateral substantia gelatinosa following unilateral peripheral nerve injury. This increase in synaptic size may reflect an activity-dependent increase in synaptic strength and efficacy, ultimately resulting in chronic neuropathic pain symptoms.

Chapter 5 describes the degree of glial activation at 3 months following sciatic nerve transection. Astroglia, but not microglia, were shown to be activated at this late phase after nerve injury. Surprisingly, the degree of astroglial activation was inversely related to the degree of mechanical hypersensitivity, suggesting that astroglia display anti-nociceptive properties at chronic stages of neuropathic pain.

In Chapter 6, nociceptive fiber sprouting is described within the spinal nociceptive network in the setting of $L 5$ spinal nerve transection. More specifically, sprouting responses were observed within and above the spinal layer consisting of PKCY-positive interneurons being part 
of a normally inactive circuit. Nociceptive sprouting responses were paralleled by an increase in c-Fos positive profiles within and above the PKCY-positive layer, indicating the activation of the normally inactive circuit. Moreover, we have shown that partial inhibition of microglial activation resulted in attenuated mechanical hypersensitivity but failed to decrease the observed sprouting responses. 


\section{Samenvatting}

Hoofdstuk 1 geeft een kort overzicht aangaande de omvang van het klinisch probleem van neuropathische pijn en een beschrijving van de diermodellen die gebruikt werden voor de experimenten beschreven in dit proefschrift. Daarnaast worden de onderzoeksvragen en doelstellingen van dit proefschrift beschreven.

Hoofdstuk 2 geeft een samenvatting van de literatuur aangaande mechanismen die bijdragen aan neuropathische pijn. Binnen dit hoofdstuk wordt er speciale aandacht besteed aan neuronale en niet-neuronale factoren die bijdragen aan de onbehandelbare en chronische eigenschappen van neuropathische pijn.

De mate waarin motorische deficieten optreden na transectie van de nervus ischiadicus staat beschreven in hoofdstuk 3. Aangezien ernstige motorische afwijkingen kunnen interfereren met de waarden verkregen uit de pijntesten, die afhankelijk zijn van terugtrekreflexen, kunnen de resultaten beschreven in dit hoofdstuk van groot belang zijn voor de interpretatie van de pijndata in dit diermodel van chronische neuropathische pijn. We mogen concluderen dat de waargenomen veranderingen in motorische functies na transectie van de nervus ischiadicus suggereren dat geen van de motorische deficieten zouden interfereren met terugtrekreflexen. Daarom is het model van transectie van de nervus ischiadicus een goed model om pijnresponsen te meten en bijgevolg de mechanismen die bijdragen aan chronische neuropatische pijn te onderzoeken.

Hoofdstuk 4 beschrijft de synaptische veranderingen aanwezig in chronische neuropathische pijn. Transectie van de nervus ischiadicus resulteert in chronische neuropathische pijn die minstens 3 maanden na het letsel aanhoudt. Op dit tijdstip werden synaptische veranderingen waargenomen in de vorm van veranderingen in de grootte van de synapsen. Immunohistochemische analyses voor het eiwit synaptophysine heeft aangetoond dat het aantal grote synapsen toegenomen waren in aantal in de ipsilaterale substantia gelatinosa na perifeer zenuwletsel. Deze toename in synapsgrootte kan een weergave zijn van een activeits-afhankelijke toename in synaptische sterkte en efficiëntie, uiteindelijk resulterend in chronische neuropathische pijn.

Hoofdstuk 5 behandelt de mate van glia activatie op 3 maanden na transectie van de nervus ischiadicus. Astroglia, maar niet microglia, bleken geactiveerd te zijn in deze late fase na zenuwletsel. De mate van astroglia activatie bleek omgekeerd gerelateerd te zijn aan de mate van mechanische hypersensitiviteit, suggererend dat astrocyten anti-pijn eigenschappen vertonen in chronische fases van neuropathische pijn. 
In hoofdstuk 6 beschrijven we groeiresponsen van nociceptieve vezels in het spinal pijnnetwerk na transectie van de L5 spinale zenuw. Deze groeiresponsen werden specifiek aangetoond in spinale lagen die gekenmerkt zijn door de aanwezigheid van PKCY-positieve interneuronen die deel uit maken van een inactief circuit. Groeiresponsen van deze nociceptieve vezels gingen gepaard met een stijging van het aantal c-Fos positieve cellen in en boven de PKCY-positieve laag, hetgeen er op wijst dat het normaal inactieve circuit nu geactiveerd is geworden. Daarbij tonen we ook aan dat gedeeltelijke onderdrukking van microglia activatie leidt tot verminderde mechanische hypersensitiviteit, maar dat dit geen effect heeft op de waargenomen groeiresponsen. 


\section{Dankwoord}

Promotiereglement Universiteit Maastricht, Hoofdstuk 2 - de promovendus, Artikel 5:

"Een verzoek tot toelating tot de promotie bevat tevens een verklaring dat het proefschrift het resultaat is van wetenschappelijk werk dat door de promovendus zelfstandig is verricht..."

Maar een proefschrift is natuurlijk nooit het werk van één persoon alleen. Het komt altijd tot stand met de hulp van anderen, die daarom een plaats verdienen in dit dankwoord.

Allereerst wil ik mijn promotor Prof. dr. Marco Marcus bedanken. Jouw kijk op de onderzoeksplannen vanuit een klinisch perspectief bracht vaak een belangrijke meerwaarde in de experimenten. Ook onze gesprekken over mijn toekomstige carrière hebben me duidelijk gemaakt in welke richting ik verder zal gaan.

Daarnaast wil ik ook mijn copromotoren Dr. Bert Joosten en Dr. Ronald Deumens bedanken.

Bert, van jou leerde ik dat de aspecten "tijd" en "plaats" in het kader van wetenschappelijk onderzoek van groot belang zijn. Als ik terugkijk naar de afgelopen 4 jaren die ik heb mogen meemaken in jouw groep, bleken de aspecten tijd en plaats ook voor mij persoonlijk van toepassing: ik heb hier een zeer mooie "tijd" beleefd, in zekere mate bepaald door de "plaats" waar ik mijn onderzoek heb mogen doen.

Ronald, jouw inbreng tijdens mijn onderzoek is ontzettend belangrijk geweest. Door je grote enthousiasme was je altijd bereid om mijn vragen te beantwoorden en te helpen op praktisch vlak waar nodig. Daarnaast wil ik je bedanken voor de leuke sfeer die er was, niet enkel op de werkvloer maar ook tijdens de congresbezoeken. Ik wens je ontzettend veel succes met je onderzoekscarrière!

Logischerwijs volgen nu de teamleden van de Joosten-groep: Michiel, Liesbeth, Sabien, Sofie, Bart, Wouter, Wiel, Anne, Joris, Helwin en Guido. Bedankt allemaal voor jullie hulp op alle vlakken. Ik vond het geweldig om jullie als collega's te hebben! Ook bedankt aan alle studenten die me geholpen hebben bij de verschillende projecten: Martin Knüwer, Ryanne Lemmens en Karin Davids.

Ook wil ik graag Prof. dr. Van Kleef bedanken voor zijn steun binnen mijn onderzoek. Hermina Gielen, ontzettend bedankt voor je administratieve steun, waardoor het vele papierwerk uiteindelijk zeer vlot verlopen is. 
Prof. dr. De Baets, Mario Losen, Pilar Martinez, bedankt voor de goede en aangename samenwerking. Ook van jullie heb ik veel mogen leren. Ook dank aan Prof. dr. Steinbusch omdat ik mijn onderzoek voornamelijk op uw werkvloer heb mogen uitvoeren. Ook wil ik alle analisten op deze werkvloer van harte bedanken voor hun hulp en bijdrage aan mijn labwerkzaamheden. Ook de mensen van het CPV verdienen een plaats in dit dankwoord voor hun hulp en inzet.

The first year of my PhD project was made possible by Dr. Gary Brook and Dr. Armin Buss, who hired me to work on a project at the RWTH in Aachen. During this project, I received lots of help form Dr. Jörg Mey. Thank you, Jörg, for offering me a place in your lab, your practical assistance and the discussions.

Ook wil ik mijn (toekomstige) schoonfamilie bedanken voor de interesse die ze steeds getoond hebben in mijn onderzoek.

Phie, lieverd, gij waart mijn belangrijkste motivator. Ik ben blij dat ge er altijd waart om me te steunen tijdens mijn promotieonderzoek. Ik kijk ontzettend uit naar onze toekomst samen en hoop dat we nog vele mooie momenten mogen meemaken!

Mama, Papa, zonder jullie had ik nooit gestaan waar ik op dit moment sta. Zonder jullie bijdrage, op alle vlakken, had dit proefschrift er niet gelegen. Jullie toonden steeds oprechte interesse in mijn onderzoek en waren ook altijd bereid te luisteren naar de hoogtes en laagtes die er waren. Ik ben ontzettend blij dat jullie er voor me zijn! Dank jullie wel voor alles!

Ook al heb je je naam niet teruggevonden in dit dankwoord, ik ben er zeker van dat je toch een bijdrage geleverd hebt aan mijn proefschrift. Sfeer op de werkvloer, advies, praktische hulp, een leuke babbel, het maakt niet uit: bedankt daarvoor! 


\section{Curriculum Vitae}

Robby Jean Paul Jaken werd geboren op 17 juni 1984 te Bilzen en groeide op in Zutendaal (België). In 2002 studeerde hij af aan het Heilig-Hart College in Lanaken in de richting Moderne Talen - Wiskunde. In datzelfde jaar startte hij zijn universitaire studies Biomedische Wetenschappen aan het toenmalige Limburgs Universitair Centrum (nu Universiteit Hasselt). In 2006 behaalde hij zijn Master diploma in de Biomedische Wetenschappen met afstudeerrichting "Klinische Moleculaire Levenswetenschappen". Tijdens het laatste jaar van deze universitaire studies volgde hij zijn masterstage aan de afdeling Psychiatrie en Neuropsychologie aan de Universiteit Maastricht binnen de groep van Dr. Bert Joosten. In de maand september van 2006 startte hij als promovendus binnen het Pain Management and Research Center van de afdeling Anesthesiologie aan de Universiteit Maastricht. Tijdens het eerste jaar van dit traject was hij werkzaam aan de RWTH in Aken (Duitsland) onder supervisie van Dr. Jörg Mey, Dr. Gary Brook en Dr. Armin Buss. De daaropvolgende drie jaar was zijn werkterrein de afdeling Anesthesiologie aan het Maastricht University Medical Center in Maastricht (Nederland). Tijdens deze 4 jaar onderzocht hij de mogelijke mechanismen die bijdragen aan de ontwikkeling en het onderhoud van pijn ten gevolgde van perifere zenuwschade. De resultaten van dit onderzoek zijn te lezen in dit proefschrift. Aan het begin van zijn traject als promovendus ontving hij een Kootstra Fellowship voor talentvolle student aan de Universiteit Maastricht.

Robby Jean Paul Jaken was born on June 17, 1984 in Bilzen and grew up in Zutendaal (Belgium). In 2002 he graduated at Heilig-Hart College in Lanaken. In the same year, he started his study in Biomedical Sciences at the University of Hasselt. In 2006 he obtained his degree of Master in Biomedical Sciences, specialty Clinical Molecular Life Sciences. During the last year of this study, he did his internship at the department Psychiatry and Neuropsychology at the University of Maastricht within the group of Dr. Bert Joosten. In 2006, he started as a PhD student at the Pain Management and Research Center of the Department of Anesthesiology at the University of Maastricht. During these 4 years, he investigated the mechanisms involved in the development and chronicity of peripheral nerve injury induced - pain. The results of this PhD project are described in this thesis. In the beginning of his PhD project, he was awarded a Kootstra Fellowship for talented student at the University of Maastricht. 
144 | Page 


\section{List of publications}

\section{Peer-reviewed publications}

Jaken RJ, Joosten EA, Knüwer M, Miller R, van der Meulen I, Marcus MA, Deumens R. Synaptic plasticity in the substantia gelatinosa in a model of chronic neuropathic pain. Neurosci Lett. 2010 Jan 18;469(1):30-33.

Deumens R, Jaken RJ, Knaepen L, van der Meulen I, Joosten EA. Inverse relation between intensity of GFAP expression in the substantia gelatinosa and degree of chronic mechanical allodynia. Neurosci Lett. 2009 Mar 13;452(2):101-5.

Deumens R, Jaken RJ, Marcus MA, Joosten EA. The CatWalk gait analysis in assessment of both dynamic and static gait changes after adult rat sciatic nerve resection. J Neurosci Methods. 2007 Aug 15;164(1):120-30.

Deumens R, Lubbers M, Jaken RJ, Meijs MF, Thurlings RM, Honig WM, Schachner M, Brook GA, Joosten EA. Mice lacking $L 1$ have reduced CGRP fibre in-growth into spinal transection lesions. Neurosci Lett. 2007 Jun 15;420(3):277-281.

Deumens R, Koopmans GC, Jaken RJ, Morren K, Comhair T, Kosar S, Steinbusch HWM, Den Bakker CGJ, Joosten EAJ. Stimulation of neurite outgrowth on neonatal cerebral astrocytes is enhanced in the presence of BDNF. Neurosci Lett. 2006 Oct 30;407(3):268-73

\section{Publications in progress}

Jaken RJ, van Gorp SF, Joosten EA, Martínez-Martínez P, Losen M, De Baets MH, Marcus MA, Deumens R. Nociceptive fiber sprouting and neuropathic pain: activation of a silent circuit? (Resubmitted to Pain)

Jaken RJ*, Knaepen L* Berger J, Janssen SP, Marcus MA, Joosten EA, Deumens R. Cellular and molecular insights into the chronic and intractable nature of neuropathic pain.

(Submitted) 


\section{Abstracts - oral presentations}

Jaken RJ, Knaepen L, Marcus MA, Joosten EA, Deumens R. Astroglia and synapses in neuropathic pain. (2009) Endo-Neuro-Psycho Meeting, Doorwerth (NL)

Jaken RJ, Knaepen L, van der Meulen I, Marcus MA, Joosten EA, Deumens R. Astroglial activation in the substantia gelatinosa is higher in neuropathic rats with a lower degree of chronic mechanical allodynia.

(2009) Anesthesiologendagen, Maastricht (NL)

\section{Abstracts - poster presentations (selected; first author)}

Jaken RJ, Marcus MA, Joosten EA, Deumens R. Spatio-temporal rewiring of the spinal nociceptive network in a rat model of neuropathic pain.

(2010) NeuPSIG $3^{\text {rd }}$ International Congress on Neuropathic Pain, Athens (GR)

Jaken RJ, Knaepen L, van der Meulen I, Marcus M, Joosten E, Deumens R. Chronic neuropathic pain: structural changes in the superficial dorsal spinal cord.

(2009) NvA 6de wetenschapsdag, Ede (NL)

Jaken RJ, Knaepen L, van der Meulen I, Marcus M, Joosten E, Deumens R. Astrocyte activation in chronic neuropathic pain: good or bad?

(2009) NvA Anesthesiologendagen, Maastricht (NL)

Jaken RJ, Knaepen L, van der Meulen I, Marcus M, Joosten E, Deumens R. Astroglial activation in the substantia gelatinosa is higher in neuropathic rats with a lower degree of chronic mechanical allodynia.

(2009) $8^{\text {th }}$ Dutch Endo-Neuro-Psycho Meeting, Doorwerth (NL)

Jaken RJ, Knüwer M, Joosten EA, Marcus MA, Deumens R. Synaptic plasticity in the substantia gelatinosa and the development of chronic neuropathic pain following peripheral nerve injury. (2009) Brain \& Behaviour Instituutsdag, Maastricht, (NL)

Jaken RJ, Knaepen L, van der Meulen I, Marcus M, Joosten E, Deumens R. Astroglial activation in the substantia gelatinosa is higher in neuropathic rats with a lower degree of chronic mechanical allodynia.

(2008) $7^{\text {th }}$ Dutch Endo-Neuro-Psycho Meeting, Doorwerth (NL) 
Jaken RJ, Knüwer M, Joosten EA, Marcus MA, Deumens R. Synaptic plasticity in the substantia gelatinosa and the development of chronic neuropathic pain following sciatic nerve resection.

(2008) NeuPSIG satellite to the Glasgow World Congress on Pain, London (UK)

Jaken RJ, Gabriel AF, Koopmans GC, van Kleef M, Marcus MA, Joosten EA, Deumens R. Innovative use of the CatWalk for detection of gait changes after rat spinal cord injury and inflammatory pain. (2007) Annual Society for Neuroscience Meeting, San Diego (USA)

Jaken RJ, Gabriel AF, Joosten EA, Koopmans GC, Deumens R. Assessment of neuropathic pain and locomotor deficits using the CatWalk gait analysis.

(2007) NeuPSIG 2nd International Congress on Neuropathic Pain, Berlin (D)

Jaken RJ, Gabriel AF, Joosten EA, Koopmans GC, Deumens R. The CatWalk gait analysis as a valuable tool to assess both interlimb coordination and pain-induced gait adaptations in rodents. (2006) $6^{\text {th }}$ International Symposium on Experimental Spinal Cord Repair and Regeneration, Brescia (I)

Jaken RJ, Gerver J, Deumens R, Honig WM, Hamers FP, Joosten EA, Koopmans GC. A quantitative study of normal locomotion in Wistar, Lewis and Sprague-Dawley rats: Differential effects on strain and walking velocity as assessed by the CatWalk.

(2006) $5^{\text {th }}$ Dutch Endo-Neuro-Psycho Meeting, Doorwerth (NL) 
